\title{
Dynamics of epidemic spreading on connected graphs
}

\author{
Christophe Besse $^{1} \cdot$ Grégory Faye $^{1}[$
}

Received: 23 November 2020 / Revised: 3 March 2021 / Accepted: 26 March 2021 /

Published online: 16 April 2021

๑) The Author(s), under exclusive licence to Springer-Verlag GmbH Germany, part of Springer Nature 2021

\begin{abstract}
We propose a new model that describes the dynamics of epidemic spreading on connected graphs. Our model consists in a PDE-ODE system where at each vertex of the graph we have a standard SIR model and connections between vertices are given by heat equations on the edges supplemented with Robin like boundary conditions at the vertices modeling exchanges between incident edges and the associated vertex. We describe the main properties of the system, and also derive the final total population of infected individuals. We present a semi-implicit in time numerical scheme based on finite differences in space which preserves the main properties of the continuous model such as the uniqueness and positivity of solutions and the conservation of the total population. We also illustrate our results with a collection of numerical simulations for a selection of connected graphs.
\end{abstract}

Keywords SIR model · Graph · Diffusion equation

Mathematics Subject Classification 34D05 $\cdot 35 \mathrm{Q} 92 \cdot 35 \mathrm{~B} 40 \cdot 92-10 \cdot 92 \mathrm{D} 30$

\section{Introduction}

Classical SIR compartment models are cornerstone models of epidemiology which allow one to study the evolution of an infected population at a given spatial scale (e.g. whole countries, regions, counties or cities). Such models date back to the pioneer work of Kermack and McKendrick (1927) and describe the evolution of susceptible (S) and infected (I) populations which eventually become removed (R) via systems of ordinary differential equations which typically take the form

Grégory Faye

gregory.faye@math.univ-toulouse.fr

1 CNRS, UMR 5219, Institut de Mathématiques de Toulouse, 31062 Toulouse Cedex, France 


$$
\left\{\begin{aligned}
S^{\prime}(t) & =-\tau S(t) I(t), \\
I^{\prime}(t) & =\tau S(t) I(t)-\eta I(t), \\
R^{\prime}(t) & =\eta I(t),
\end{aligned}\right.
$$

where $\tau>0$ is a contact rate between susceptible and infected populations, and $1 / \eta>$ 0 is the average infectious period; see Hethcote (2000) for a review on SIR models. These models have been used in the past to reproduce data of epidemic outbreaks such as the bubonic plague (Kermack and McKendrick 1927), malaria (Mandal et al. 2011), SARS influenza (Centers for Disease Control and Prevention 2003; Magal et al. 2016) and most recently COVID-19 (New England Journal of Medicine 2020; Magal and Webb 2018; Liu et al. 2020); see also Stolerman et al. (2015) and Magal and Webb (2018) for other applications.

In classical SIR models such as (1.1), interactions among the infected population are oversimplified, and when taken into account they typically involve transfer matrices of populations of infected between various uniform patches (Van den Driessche and Watmough 2002; Magal et al. 2016, 2018). Our interest lies in the understanding of the intricate interplay between spatial effects and heterogeneous interactions among infected populations. Schematically, we propose a model composed of cities linked by a given transportation network (roads, railroads or rivers), see Fig. 1 for an illustration in the case of France. It will turn out that the appropriate theoretical framework will be graph theory where each vertices of the graph will be thought as the cities and the edges the lines of transportation. In a first approximation, we will assume that infected populations are only subject to spatial diffusion along the lines, as it is traditionally assumed in classical spatial SIR models (Aronson 1977; Diekmann 1978; Reluga 2004; Berestycki et al. 2020). As a consequence, in our model, the dynamics of the epidemic only takes place in the cities. Interactions are then modeled by flux exchanges between cities and lines where we assume that some fraction of infected individuals can either leave a city to be on a line, or leave a line and stop in a city, or pass from one line to another through a city. The typical question that we address here can easily be stated as follows. Given a connected graph of cities linked by roads and an initial configuration of infected individuals, how does the epidemic spread into the network and what is the eventual final configuration of the infected population? Our aim here is to gain insight into this spreading aspect at the fundamental mathematical level of a SIR type model that incorporates the possibility of infected individuals to travel along a specific given transportation network.

Our framework is at the crossroad of models that take into account lines of transportation such as recent reaction-diffusion models that study propagation of epidemics along lines with fast diffusion (Berestycki et al. 2020) and models that incorporate networks with more sophisticated interactions dynamics (Britton et al. 2008; Sahneh et al. 2013; Spricer and Britton 2019; Ball and Britton 2020; Bonnasse-Gahot et al. 2018). On a formal level, our proposed model can be thought of as being a one-dimensional version of the planar reaction-diffusion system of Berestycki et al. (2020) with a line of fast diffusion in the case of one city and one line of transportation. Actually, the graph structure of the transportation network provides a natural embedding into a planar spatial domain. From a mathematical point of view, our model shares also 
Fig. 1 Map of France with an illustration of a connected graph connecting major cities

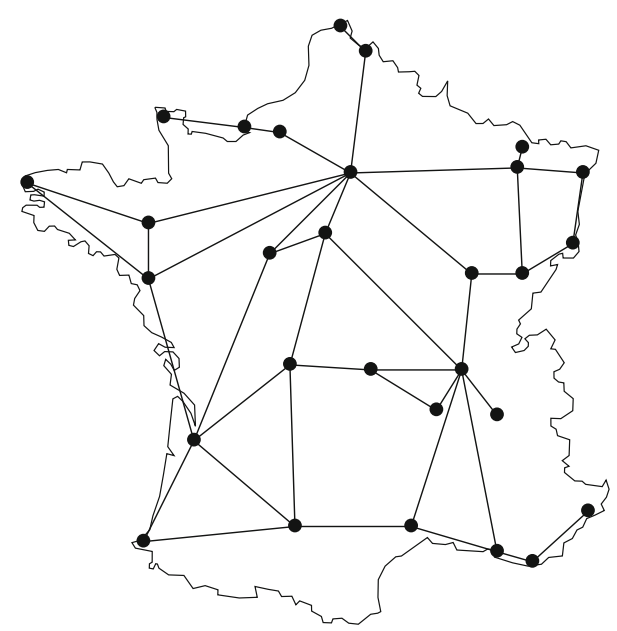

some similarities with the PDE-ODE model of David et al. (2020) which studies the spread of airborne diseases where the movement of pathogens in the air is assumed to follow a linear diffusion. Actually, one-dimensional PDE-ODE models have been successfully used in other biological contexts, and we refer to the line of works (Gou and Ward 2016; Gou et al. 2015; Paquin-Lefebvre et al. 2020) on cell-bulk models of cell signaling. Although these models share some similarities, the long time dynamics are different. In Gou and Ward (2016), Gou et al. (2015) and Paquin-Lefebvre et al. (2020), one typically observes oscillatory dynamics caused by the coupling at the boundary via the loss of stability of steady-states through Hopf bifurcations, whereas the long time behavior of the solutions of our model presents the characteristics of SIR compartment models with the convergence to a steady-state of the system. As it will be demonstrated, the selected steady-state is entirely determined by the initial configuration of individuals in our network.

Finally, we highlight that there is an intrinsic difference between the PDE-ODE model that we propose and the compartment models on fixed graphs that are prominent in the literature on the subject, see for example (Britton et al. 2008; Sahneh et al. 2013; Stolerman et al. 2015; Spricer and Britton 2019; Ball and Britton 2020; BonnasseGahot et al. 2018) and references therein. In our setting, connections between vertices are not instantaneous as infected individuals have to diffuse along edges in order to be transported from one vertex to another. As a consequence, our PDE-ODE model intrinsically incorporates very subtle nonlocal in time behaviors that typical compartment models on fixed graph with local interactions do not take into account.

\section{Model formulation and main results}

Throughout, we denote by $\mathcal{G}=(\mathcal{V}, \mathcal{E})$ a compact metric graph, i.e. a collection of vertices $\mathcal{V}$ and edges $\mathcal{E}$ and further assume that $\mathcal{G}$ is finite and connected. Each edge $e \in \mathcal{E}$ is identified with a segment $\Omega_{e}=\left[0, \ell_{e}\right]$ with $\ell_{e} \in(0, \infty)$, where $\ell_{e}$ is the 
finite length of the edge. A real valued function $u: \mathcal{G} \longrightarrow \mathbb{R}$ is a collection of one dimensional maps defined for each edge $e \in \mathcal{E}$ :

$$
u_{e}: \Omega_{e} \longrightarrow \mathbb{R}
$$

For future references, we define $B C(\mathcal{G}, \mathbb{R})$ the space of bounded continuous functions on $\mathcal{G}$ as

$$
B C(\mathcal{G}, \mathbb{R}):=\bigoplus_{e \in \mathcal{E}} B C\left(\Omega_{e}, \mathbb{R}\right)
$$

and similarly $B C^{k}(\mathcal{G}, \mathbb{R})$ with $k \geq 1$. We define the $L^{\infty}$ norm on $\mathcal{G}$ for $u \in B C(\mathcal{G}, \mathbb{R})$ as

$$
\|u\|_{\infty}:=\max _{e \in \mathcal{E}} \sup _{x \in \Omega_{e}}\left|u_{e}(x)\right| .
$$

\subsection{A SIR model on compact connected graph}

Given a graph $\mathcal{G}$, we let $X_{v}(t):=\left(S_{v}(t), I_{v}(t), R_{v}(t)\right) \in \mathbb{R}^{3}$, for each $v \in \mathcal{V}$, where $S_{v}(t)$ represents the population of susceptible individuals, $I_{v}(t)$ the population of infected individuals and $R_{v}(t)$ the population of removed individuals at vertex $v \in \mathcal{V}$ and time $t>0$. We assume that $X_{v}$ evolves according to a SIR model of the form

$$
\left\{\begin{array}{l}
S_{v}^{\prime}(t)=-\tau_{v} S_{v}(t) I_{v}(t) \\
I_{v}^{\prime}(t)=\tau_{v} S_{v}(t) I_{v}(t)-\eta_{v} I_{v}(t)+\sum_{e \sim v} \alpha_{e}^{v} u_{e}(t, v)-\bar{\lambda}_{v} I_{v}(t) \\
R_{v}^{\prime}(t)=\eta_{v} I_{v}(t)
\end{array}\right.
$$

where $\tau_{v}, \eta_{v}>0$ are the intrinsic parameters of the epidemic which may depend on the vertex $v$. The contribution $-\bar{\lambda}_{v} I_{v}(t)$ in the right-hand side of the equation for the infected population encodes the fact that infected individuals can leave the vertex $v$ to incident edges whereas $\sum_{e \sim v} \alpha_{e}^{v} u_{e}(t, v)$ reflects the contribution of incoming infected individuals from incident edges. Here, $e \sim v$ denotes the edges incident to the vertex $v$ and

$$
\bar{\lambda}_{v}:=\sum_{e \sim v} \lambda_{e}^{v}
$$

such that $\lambda_{e}^{v} I_{v}(t)$ infected individuals leave vertex $v$ to edge $e$. We have assumed that only the infected population is subject to movement, and we think of $S_{v}$ being an ambient population whose movement does not affect its distribution. We recover the standard SIR model (1.1) by considering the trivial graph $\mathcal{G}=(\{v\}, \emptyset)$. Throughout the manuscript, we will assume the following standing hypothesis on the coefficients $\alpha_{e}^{v}$ and $\lambda_{e}^{v}$ in (2.1). 
Hypothesis 2.1 For each $(e, v) \in \mathcal{E} \times \mathcal{V}$ we assume that

$$
\alpha_{e}^{v} \in(0,1) \quad \text { and } \quad \lambda_{e}^{v} \in(0,1)
$$

together with

$$
\sum_{e \sim v} \lambda_{e}^{v} \in(0,1) \quad \text { and } \quad \sum_{e \sim v} \alpha_{e}^{v} \in(0,1)
$$

Next, for each $e \in \mathcal{E}$, we let $d_{e}>0$ and we assume that $u_{e}$ evolves according to

$$
\partial_{t} u_{e}(t, x)=d_{e} \partial_{x}^{2} u_{e}(t, x), \quad t>0, \quad x \in \stackrel{\circ}{\Omega}_{e}
$$

Assuming that infected individuals have local diffusion along the edges of the graph is a first approximation, and this can be viewed as a limiting Brownian movement of individuals. Possible extensions could be to incorporate nonlocal diffusion or transport terms.

It now remains to model the exchanges of infected individuals at the vertices. For each $v \in \mathcal{V}$, we associate an integer $\delta_{v} \geq 1$ which we refer to as its degree (i.e. number of edges incident to the vertex $v$ ). We define $\mathbf{u}_{v}(t) \in \mathbb{R}^{\delta_{v}}$ as the column vector function

$$
\mathbf{u}_{v}(t):=\left(u_{e}(t, v)\right)_{e \sim v}
$$

where we recall that $e \sim v$ denotes the edges incident to the vertex $v$, and thus $u_{e}(t, v)$ is the corresponding limit value of $u_{e}$ at $x=v$. Define also $\partial_{n} \mathbf{u}_{v}(t) \in \mathbb{R}^{\delta_{v}}$ as the column vector function

$$
\partial_{n} \mathbf{u}_{v}(t):=\left(\partial_{n} u_{e}(t, v)\right)_{e \sim v},
$$

where $\partial_{n} u_{e}(t, v)$ is the outwardly normal derivative of $u_{e}$ at the vertex $v$. Our boundary conditions at the vertex $v$ that link (2.1) and (2.2) are described by

$$
D_{v} \partial_{n} \mathbf{u}_{v}(t)+K_{v} \mathbf{u}_{v}(t)=\Lambda_{v} \mathbf{I}_{v}(t)
$$

where $D_{v} \in \mathscr{M}_{\delta_{v}}(\mathbb{R})$ is the diagonal matrix $D_{v}=\operatorname{diag}\left[\left(d_{e}\right)_{e \sim v}\right]$ and $K_{v} \in \mathscr{M}_{\delta_{v}}(\mathbb{R})$ whose structure will be specified below. Formally, (2.3) encodes the balance of fluxes of infected individuals at the vertex $v$, and we will demonstrate this heuristic rigorously by showing in the forthcoming Sect. 2.4 the conservation of total population.

\subsection{Assumptions on the connectivity matrices $K_{V}$}

We now precise the form of the matrix $K_{v}$ entering in the boundary condition (2.3). Essentially, $K_{v}$ gathers two contributions. One contribution comes from the exchanges between infected individuals at the vertex with the incoming infected individuals for 
the incident edges. The second contribution models exchanges between edges. Indeed we allow infected individuals to pass from one edge to another one. More precisely, we have that $K_{v}$ splits into two parts

$$
K_{v}:=A_{v}+N_{v}
$$

where the matrix $A_{v} \in \mathscr{M}_{\delta_{v}}(\mathbb{R})$ is the diagonal matrix $A_{v}=\operatorname{diag}\left(\alpha_{e}^{v}\right)_{e \sim v}$ while the matrix $N_{v} \in \mathscr{M}_{\delta_{v}}(\mathbb{R})$ is such that the sum of each column is zero. More precisely, if we label by $e \sim v=\left(e_{1}, \ldots, e_{\delta_{v}}\right)$ the edges incident to the vertex $v$, we have that for all $i=1, \ldots, \delta_{v}$

$$
\left(N_{v}\right)_{i, i}=\sum_{j \neq i} v_{e_{i}, e_{j}}^{v}
$$

and for $i \neq j=1, \ldots, \delta_{v}$

$$
\left(N_{v}\right)_{i, j}=-v_{e_{j}, e_{i}}^{v}
$$

In the case $\delta_{v}=3$, we get

$$
N_{v}=\left(\begin{array}{ccc}
v_{e_{1}, e_{2}}^{v}+v_{e_{1}, e_{3}}^{v} & -v_{e_{2}, e_{1}}^{v} & -v_{e_{3}, e_{1}}^{v} \\
-v_{e_{1}, e_{2}}^{v} & v_{e_{2}, e_{1}}^{v}+v_{e_{2}, e_{3}}^{v} & -v_{e_{3}, e_{2}}^{v} \\
-v_{e_{1}, e_{3}}^{v} & -v_{e_{2}, e_{3}}^{v} & v_{e_{3}, e_{1}}^{v}+v_{e_{3}, e_{2}}^{v}
\end{array}\right)
$$

see Fig. 2 for an illustration in that case.

Furthermore, for the diagonal term we will use the shorthand notation

$$
\left(N_{v}\right)_{e, e}=\sum_{e^{\prime} \neq e} v_{e, e^{\prime}}^{v}
$$

The fact that $N_{v} \in \mathscr{M}_{\delta_{v}}(\mathbb{R})$ is such that the sum of each column is zero precisely encodes the fact that there is the conservation of infected individuals through exchanges between incident edges at each vertex. And, we remark that it implies that the matrix $K_{v}$ has a strict column diagonal dominance in the sense that for each $i=1, \ldots, \delta_{v}$

$$
\sum_{j=1}^{\delta_{v}}\left(K_{v}\right)_{e_{j}, e_{i}}=\alpha_{e_{i}}^{v}>0
$$

because of this specific structure of $N_{v}$. From now on we also assume that $K_{v}$ has a diagonal dominance with respect to its lines. This property will be crucial later on in the proof of existence of solutions. As a consequence, we impose the following running assumptions on the matrices $K_{v}$.

Hypothesis 2.2 For each $v \in \mathcal{V}$ and $\left(e, e^{\prime}\right) \in \mathcal{E} \times \mathcal{E}$, we assume that

$$
v_{e, e^{\prime}}^{v} \in[0,1)
$$




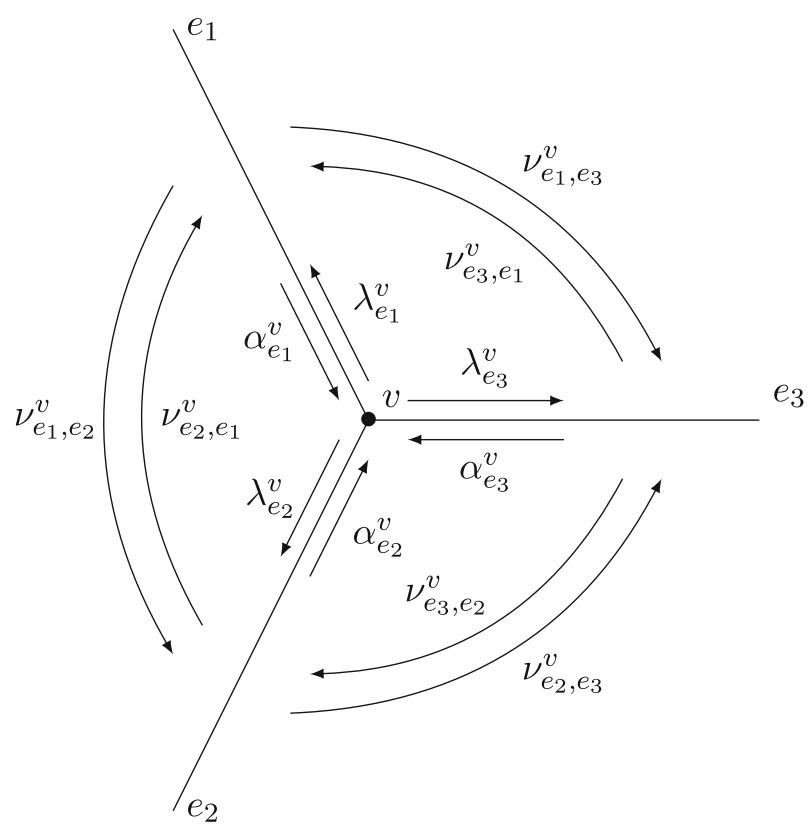

Fig. 2 Schematic illustration of the exchanges at a given vertex $v$ with $\delta_{v}=3$

Furthermore, we impose that for all $v \in \mathcal{V}$

$$
\sum_{e^{\prime} \neq e} v_{e^{\prime}, e}^{v}<\alpha_{e}^{v}+\sum_{e^{\prime} \neq e} v_{e, e^{\prime}}^{v}
$$

together with

$$
\left(K_{v}\right)_{e, e}:=\alpha_{e}^{v}+\sum_{e^{\prime} \neq e} v_{e, e^{\prime}}^{v} \in(0,1)
$$

for each $e \sim v$.

Remark 2.3 If the exchanges between the edges are symmetric, that is for each $v \in \mathcal{V}$ the matrices $N_{v}$ are symmetric, that is

$$
v_{e, e^{\prime}}^{v}=v_{e^{\prime}, e}^{v}, \quad \forall\left(e, e^{\prime}\right) \in \mathcal{E} \times \mathcal{E},
$$

then Hypothesis 2.2 is automatically satisfied.

\subsection{Initial configuration}

Finally, we complement our coupled PDE-ODE (2.1)-(2.2)-(2.3) with some initial conditions. We assume that at $t=0$, we have 


$$
u(t=0, \cdot)=u^{0} \in B C(\mathcal{G}, \mathbb{R})
$$

such that for $e \in \mathcal{E}$,

$$
u_{e}^{0}(x) \geq 0, \quad x \in \Omega_{e} .
$$

On the other hand, for the ODE system (2.1), we suppose that

$$
\left(S_{v}(t=0), I_{v}(t=0), R_{v}(t=0)\right)=\left(S_{v}^{0}, I_{v}^{0}, 0\right) \geq \mathbf{0}, \quad \forall v \in \mathcal{V}
$$

We further assume that (2.3) is satisfied at $t=0$

$$
D_{v} \partial_{n} \mathbf{u}_{v}^{0}+K_{v} \mathbf{u}_{v}^{0}=\Lambda_{v} \mathbf{I}_{v}^{0}
$$

with obvious notations $\mathbf{u}_{v}^{0}:=\left(u_{e}^{0}(v)\right)_{e \sim v}$ and $\mathbf{I}_{v}^{0}:=\left(I_{v}^{0}, \ldots, I_{v}^{0}\right)^{\mathbf{t}}$. Last, we impose that the initial total population of infected individuals is strictly positive,

$$
\sum_{v \in \mathcal{V}} I_{v}^{0}>0
$$

and that susceptible individuals are initially present at each vertex of the graph

$$
S_{v}^{0}>0, \quad \forall v \in \mathcal{V}
$$

This in turn implies that the total population is initially

$$
M^{0}:=\sum_{e \in \mathcal{E}} \int_{\Omega_{e}} u_{e}^{0}(x) \mathrm{d} x+\sum_{v \in \mathcal{V}}\left(S_{v}^{0}+I_{v}^{0}\right)>0 .
$$

\subsection{Conservation of total population}

Assuming that there is a solution to $\left(u,\left(X_{v}\right)_{v \in \mathcal{V}}\right)$ to $(2.1)-(2.2)-(2.3)$, we have that the total mass of the system $M(t)$ defined as

$$
M(t):=\sum_{e \in \mathcal{E}} \int_{\Omega_{e}} u_{e}(t, x) \mathrm{d} x+\sum_{v \in \mathcal{V}}\left(S_{v}(t)+I_{v}(t)+R_{v}(t)\right)
$$

is a conserved quantity and thus independent of $t$.

To see that, we first remark that

$$
\left(S_{v}^{\prime}(t)+I_{v}^{\prime}(t)+R_{v}^{\prime}(t)\right)=\sum_{e \sim v} \alpha_{e}^{v} u_{e}(t, v)-\bar{\lambda}_{v} I_{v}(t)=\left\langle A_{v} \mathbf{u}_{v}(t), \mathbf{1}_{\delta_{v}}\right\rangle-\left\langle\Lambda_{v} \mathbf{I}_{v}(t), \mathbf{1}_{\delta_{v}}\right\rangle
$$

with

$$
\mathbf{1}_{\delta_{v}}:=(1, \ldots, 1)^{\mathbf{t}} \in \mathbb{R}^{\delta_{v}}
$$


and $\langle\cdot, \cdot\rangle$ is the standard Euclidean inner product on $\mathbb{R}^{\delta_{v}}$. On the other hand let us define

$$
m(t):=\sum_{e \in \mathcal{E}} \int_{\Omega_{e}} u_{e}(t, x) \mathrm{d} x
$$

and assume that $u$ is a classical solution of (2.2), which we will prove in the next section, and compute

$$
\begin{aligned}
m^{\prime}(t) & =\sum_{e \in \mathcal{E}} \int_{\Omega_{e}} \partial_{t} u_{e}(t, x) \mathrm{d} x=\sum_{e \in \mathcal{E}} d_{e}\left[\partial_{x} u_{e}(t, x)\right]_{\partial \Omega_{e}} \\
& =\sum_{v \in \mathcal{V}}\left\langle D_{v} \partial_{n} \mathbf{u}_{v}(t), \mathbf{1}_{\delta_{v}}\right\rangle \\
& =\sum_{v \in \mathcal{V}}\left\langle\Lambda_{v} \mathbf{I}_{v}(t)-K_{v} \mathbf{u}_{v}(t), \mathbf{1}_{\delta_{v}}\right\rangle \\
& =\sum_{v \in \mathcal{V}}\left\langle\Lambda_{v} \mathbf{I}_{v}(t)-A_{v} \mathbf{u}_{v}(t), \mathbf{1}_{\delta_{v}}\right\rangle-\underbrace{\sum_{v \in \mathcal{V}}\left\langle N_{v} \mathbf{u}_{v}(t), \mathbf{1}_{\delta_{v}}\right\rangle}_{=0} \\
& =-\sum_{v \in \mathcal{V}}\left(S_{v}^{\prime}(t)+I_{v}^{\prime}(t)+R_{v}^{\prime}(t)\right) .
\end{aligned}
$$

The fact that

$$
\sum_{v \in \mathcal{V}}\left\langle N_{v} \mathbf{u}_{v}(t), \mathbf{1}_{\delta_{v}}\right\rangle=0
$$

is a direct consequence on the specific structure of each matrix $N_{v}$ and the fact that the sum of each column is zero. We therefore conclude that $M^{\prime}(t)=0$ and

$$
\sum_{e \in \mathcal{E}} \int_{\Omega_{e}} u_{e}(t, x) \mathrm{d} x+\sum_{v \in \mathcal{V}}\left(S_{v}(t)+I_{v}(t)+R_{v}(t)\right)=M^{0}, \quad \forall t \geq 0
$$

Biological interpretation. Our model is thus consistent with the conservation of the total population as it is traditionally the case for SIR model in the case of zero natality/mortality rate. The exchanges between the vertices and the edges exactly compensate each other as is natural.

\subsection{Main results and outline of the paper}

We now present our main results regarding our model (2.1)-(2.2)-(2.3). At this stage of the presentation, we remain formal and refer to the following sections for precise statements and assumptions. 
Main result 1: Existence and uniqueness of classical solutions. We prove in Theorem 1 below that for each well prepared initial condition our model (2.1)-(2.2)-(2.3) admits a unique positive classical solution which is global in time. We remark that the system (2.1)-(2.2)-(2.3) is not standard as it couples a system of PDEs to ODEs at each vertices through inhomogeneous Robin boundary conditions. As a consequence, the existence and uniqueness of classical solutions has to be proved. This analysis is conducted in Sect. 3.

Main result 2: Long time behavior of the solutions. We fully characterize the long time behavior of the unique solution of our model. More precisely, we prove that the final total population of infected individuals at each vertex, denoted by $\mathcal{I}_{v}^{\infty}$, is a well defined quantity: $0<\mathcal{I}_{v}^{\infty}<\infty$ for $v \in \mathcal{V}$ and $\left(\mathcal{I}_{v}^{\infty}\right)_{v \in \mathcal{V}}$ are solutions of a system of $c \mathcal{V}+1$ implicit equations, where $c \mathcal{V}$ stands for the cardinal of $\mathcal{V}$, which belong to the parametrized submanifold

$$
\sum_{v \in \mathcal{V}}\left(S_{v}^{0} e^{-\tau_{v} \mathcal{I}_{v}^{\infty}}+\eta_{v} \mathcal{I}_{v}^{\infty}\right)=M^{0}
$$

where $M^{0}$ is the initial total mass. We refer to Theorem 2 for a precise statement. We also present further qualitative results on the final total configuration $\left(\mathcal{I}_{v}^{\infty}\right)_{v \in \mathcal{V}}$ in the fully symmetric case where we obtain closed form formula (see Lemma 4.4) and in the case of two vertices where we manage to obtain sharp bounds on the final total populations of infected individuals (see Lemma 4.5). In each case, we manage to relate these quantities to standard basic and effective reproductive number for classical SIR model. The aforementioned results are proved in Sect. 4.

Main result 3: Amass preserving semi-implicit numerical schemeMain result 3: Amass preserving semi-implicit numerical scheme. We propose and analyze a semi-implicit in time numerical scheme based on finite differences in space which has the property to preserve a discrete total mass associated to the discretization. We prove that if the time discretization constant is smaller than a universal constant depending only on the parameters of the system (and not on the space discretization constant) and if $N_{v}$ is symmetric for each $v \in \mathcal{V}$, then our mass preserving semi-implicit numerical scheme is well-posed and preserves the positivity of the solutions. We refer to Sect. 5 for a presentation of the numerical scheme and Theorem 3 for a precise statement of our main result.

Main result 4: Numerical results for various types of graphs. We illustrate our theoretical findings with selection of numerical simulations for various types of graphs in Sect. 6. We respectively study the case of 2 vertices and 1 edge, 3 vertices and 3 edges (closed graph), 4 vertices and 3 edges (star-shape graph) and $N+1$ vertices and $N$ edges with $N$ being arbitrarily large (lattice graph). Most notably, in the last case, we show the propagation of the epidemics across the vertices of the graph in the form of a traveling wave. 


\section{The Cauchy problem: existence and uniqueness of classical solutions}

This section is devoted to the proof of the following main theorem which guarantees that our model is well-posed.

Theorem 1 For each $\left(S_{v}^{0}, I_{v}^{0}\right) \geq \mathbf{0}$ with $S_{v}^{0}>0, \sum_{v \in \mathcal{V}} I_{v}^{0}>0$ and $u^{0} \in B C\left(\mathcal{G}, \mathbb{R}^{+}\right)$ that satisfy the boundary condition (2.3), there exists a unique positive global solution $\left(S_{v}, I_{v}, R_{v}\right) \in \mathscr{C}^{1}\left(\mathbb{R}^{+}, \mathbb{R}^{+} \times \mathbb{R}^{+} \times \mathbb{R}^{+}\right)$and $u \in \mathscr{C}^{1,2}\left(\mathbb{R}_{*}^{+} \times \mathcal{G}, \mathbb{R}^{+}\right)$.

The proof of Theorem 1 is divided into two parts. We first prove the existence of positive global classical solutions and then show that such constructed solutions are unique. We look for solutions that satisfy (2.1)-(2.2)-(2.3) in the classical sense, and we always assume that $\left(S_{v}^{0}, I_{v}^{0}\right) \geq \mathbf{0}$ with $S_{v}^{0}>0, \sum_{v \in \mathcal{V}} I_{v}^{0}>0$ and $u^{0} \in B C\left(\mathcal{G}, \mathbb{R}^{+}\right)$, that is for all $e \in \mathcal{E}, u_{e}^{0} \geq 0$ is bounded continuous on $\Omega_{e}$. We further assume that the initial conditions satisfy the boundary condition (2.3). We remark that the system (2.1)-(2.2)-(2.3) is not standard as it couples a system PDEs to ODEs at each vertices through inhomogeneous Robin boundary conditions. As a consequence, the wellposedness of the Cauchy problem has to be proved.

Remark 3.1 Our existence and uniqueness result extends trivially in the case that parameters $\tau_{v}>0, \alpha_{e}^{v} \in(0,1), \lambda_{e}^{v} \in(0,1)$ and $v_{e, e^{\prime}}^{v} \in[0,1)$ are continuous functions of time satisfying $\tau_{v}(t)>0, \alpha_{e}^{v}(t) \in(0,1), \lambda_{e}^{v}(t) \in(0,1)$ and $v_{e, e^{\prime}}^{v}(t) \in[0,1)$ together with Hypotheses 2.1-2.2 verified at all times $t>0$.

\subsection{Existence}

In this section, we construct a classical solution to (2.1)-(2.2)-(2.3) through a limiting argument. We will obtain a solution $\left(u,\left(X_{v}\right)_{v \in \mathcal{V}}\right)$ has the limit of a subsequence of solution $\left(\left(u^{n},\left(X_{v}^{n}\right)_{v \in \mathcal{V}}\right)\right)_{n \geq 0}$ of the following problems

$$
\left\{\begin{aligned}
\frac{\mathrm{d} S_{v}^{n}(t)}{\mathrm{d} t}= & -\tau_{v} S_{v}^{n}(t) I_{v}^{n}(t) \\
\frac{\mathrm{d} I_{v}^{n}(t)}{\mathrm{d} t}= & \tau_{v} S_{v}^{n}(t) I_{v}^{n}(t)-\left(\eta_{v}+\bar{\lambda}_{v}\right) I_{v}^{n}(t) \\
& +\sum_{e \sim v} \alpha_{e}^{v} u_{e}^{n-1}(t, v), \\
\frac{\mathrm{d} R_{v}^{n}(t)}{\mathrm{d} t}= & \eta_{v} I_{v}^{n}(t)
\end{aligned}\right.
$$

with

$$
D_{v} \partial_{n} \mathbf{u}_{v}^{n}(t)+K_{v} \mathbf{u}_{v}^{n}(t)=\Lambda_{v} \mathbf{I}_{v}^{n}(t), \quad t>0, \quad \forall v \in \mathcal{V},
$$

and

$$
\partial_{t} u_{e}^{n}(t, x)=d_{e} \partial_{x}^{2} u_{e}^{n}(t, x), \quad t>0, \quad x \in \stackrel{\circ}{\Omega}_{e}, \quad \forall e \in \mathcal{E}
$$


starting from $u^{0} \in B C\left(\mathcal{G}, \mathbb{R}^{+}\right)$and $\left(X_{v}^{0}\right)_{v \in \mathcal{V}}$. Note that (3.1)-(3.2)-(3.3) is supplemented by the same initial condition $\left(u^{0},\left(X_{v}^{0}\right)_{v \in \mathcal{V}}\right)$ at each step. We proceed along three main steps.

Step \# 1: solvability of (3.1)-(3.2)-(3.3). We first show that (3.1)-(3.2)-(3.3) admits a unique solution. It can be done by induction. Assume that at step $n-1$, we have constructed a solution $\left(u^{n-1},\left(X_{v}^{n-1}\right)_{v \in \mathcal{V}}\right)$ such that for each $t \mapsto u_{e}^{n-1}(t, v)$ is continuous, then we get the existence of a unique solution of (3.1) which is $\mathscr{C}^{1}$ in time. Next we solve the system of PDEs (3.3)-(3.2) whose coupling comes from the boundary conditions and owing that now the right-hand side of (3.2) can be seen as given inhomogeneous term of class $\mathscr{C}^{1}$ in time. As both $D_{v}$ and $K_{v}$ are invertible matrices, we get the existence of a classical solution $u^{n} \in \mathscr{C}^{1,2}$ which then ensures that $t \mapsto u_{e}^{n}(t, v)$ is continuous.

Step \#2: a priori estimates. Let $0<T<1$ be fixed. We first show by a recursive argument that $0<S_{v}^{n}, 0 \leq I_{v}^{n}, 0 \leq R_{v}^{n}$ for each $v \in \mathcal{V}$ and $0 \leq u_{e}^{n}$ for each $e \in \mathcal{E}$. It is trivial at $n=0$. Let assume that is it true at $n-1$. We start from (3.1) and a direct integration gives

$$
\begin{aligned}
S_{v}^{n}(t)= & S_{v}^{0} e^{-\tau_{v} \int_{0}^{t} I_{v}^{n}(s) \mathrm{d} s}>0, \\
I_{v}^{n}(t)= & I_{v}^{0} e^{-\left(\eta_{v}+\bar{\lambda}_{v}\right) t+\int_{0}^{t} S_{v}^{n}(s) \mathrm{d} s} \\
& +\sum_{e \sim v} \alpha_{e}^{v} \int_{0}^{t} e^{-\left(\eta_{v}+\bar{\lambda}_{v}\right)(t-s)+\int_{s}^{t} S_{v}^{n}(\tau) \mathrm{d} \tau} u_{e}^{n-1}(s, v) \mathrm{d} s \geq 0, \\
R_{v}^{n}(t)= & \eta_{v} \int_{0}^{t} I_{v}^{n}(s) \mathrm{d} s \geq 0 .
\end{aligned}
$$

Now owing that $0 \leq I_{v}^{n}$ for each $v \in \mathcal{V}$, the maximum principle implies that $u_{e}^{n} \geq 0$ for each $e \in \mathcal{E}$. Assume by contradiction that $e_{*} \in \mathcal{E}$ is the component which reaches a negative minimum, namely $u_{e_{*}}^{n}\left(t_{*}, x_{*}\right)=-\delta<0$ with $u_{e_{*}}^{n}(t, x)>-\delta$ for $t<t_{*}$ and $x \in \Omega_{e_{*}}$ and for each $e \neq e_{*}$ we have $u_{e}^{n}(t, x)>-\delta$ for $t \leq t_{*}$ and $x \in \Omega_{e}$. We know that $x_{*} \in \partial \Omega_{e_{*}}$ and let denote $v_{*}=x_{*} \in \mathcal{V}$ the vertex where this occurs. The Hopf lemma implies that $\partial_{n} u_{e_{*}}^{n}\left(t_{*}, v_{*}\right)<0$. Inspecting the boundary condition (3.2) at $v_{*}$, we obtain that

$$
\begin{aligned}
& d_{e_{*}} \partial_{n} u_{e_{*}}^{n}\left(t_{*}, v_{*}\right)+\alpha_{e_{*}}^{v_{*}} u_{e_{*}}^{n}\left(t_{*}, v_{*}\right)+\left(\sum_{e \sim e_{*}} v_{e_{*}, e}^{v_{*}}\right) u_{e_{*}}^{n}\left(t_{*}, v_{*}\right) \\
& -\sum_{e \sim e_{*}} v_{e, e_{*}}^{v_{*}} u_{e}^{n}\left(t_{*}, v_{*}\right)=\lambda_{e_{*}}^{v_{*}} I_{v_{*}}^{n}(t)
\end{aligned}
$$

which writes

$$
0>d_{e_{*}} \partial_{n} u_{e_{*}}^{n}\left(t_{*}, v_{*}\right)+\delta\left(\sum_{e \sim e_{*}} v_{e, e_{*}}^{v_{*}}-\alpha_{e_{*}}^{v_{*}}-\sum_{e \sim e_{*}} v_{e_{*}, e}^{v_{*}}\right)
$$




$$
-\sum_{e \sim e_{*}} v_{e, e_{*}}^{v_{*}}\left(\delta+u_{e}^{n}\left(t_{*}, v_{*}\right)\right)=\lambda_{e_{*}}^{v_{*}} I_{v_{*}}^{n}(t) \geq 0
$$

and leads to a contradiction. Here we have used the fact that

$$
\sum_{e \sim e_{*}} v_{e, e_{*}}^{v_{*}} \leq \alpha_{e_{*}}^{v_{*}}+\sum_{e \sim e_{*}} v_{e_{*}, e}^{v_{*}}
$$

from Hypothesis 2.2 on the matrices $\left(K_{v}\right)_{v \in \mathcal{V}}$.

Next, from the positivity of solutions, we obtain some uniform $L^{\infty}$ bounds. More precisely, we claim that there exists a constant $K>0$ depending only on $\left(T, I_{v}^{0}, S_{v}^{0},\left\|u^{0}\right\|_{\infty}\right)$ such that

$0 \leq S_{v}^{n}(t), I_{v}^{n}(t), R_{v}^{n}(t) \leq K$, and $\left|\frac{\mathrm{d} S_{v}^{n}(t)}{\mathrm{d} t}\right|,\left|\frac{\mathrm{d} I_{v}^{n}(t)}{\mathrm{d} t}\right|,\left|\frac{\mathrm{d} R_{v}^{n}(t)}{\mathrm{d} t}\right| \leq K \quad 0<t \leq T$,

and

$$
0 \leq u_{e}^{n}(t, x) \leq K, \quad 0<t \leq T, \quad x \in \Omega_{e} .
$$

First, using (3.1) we obtain that

$$
\frac{\mathrm{d} S_{v}^{n}(t)}{\mathrm{d} t}+\frac{\mathrm{d} I_{v}^{n}(t)}{\mathrm{d} t}+\frac{\mathrm{d} R_{v}^{n}(t)}{\mathrm{d} t}=-\bar{\lambda}_{v} I_{v}^{n}(t)+\sum_{e \sim v} \alpha_{e}^{v} u_{e}^{n-1}(t, v),
$$

which gives that

$$
0 \leq S_{v}^{n}(t)+I_{v}^{n}(t)+R_{v}^{n}(t) \leq S_{v}^{0}+I_{v}^{0}+T\left\|u^{n-1}\right\|_{\infty}, \quad 0<t \leq T,
$$

together with

$$
\left|\frac{\mathrm{d} S_{v}^{n}(t)}{\mathrm{d} t}\right|,\left|\frac{\mathrm{d} I_{v}^{n}(t)}{\mathrm{d} t}\right|,\left|\frac{\mathrm{d} R_{v}^{n}(t)}{\mathrm{d} t}\right| \leq C\left\|u^{n-1}\right\|_{\infty}\left(1+T+T^{2}\left\|u^{n-1}\right\|_{\infty}\right) \quad 0<t \leq T,
$$

which in turn implies that

$$
\left\|u^{n}\right\|_{\infty} \leq \tilde{C} T\left\|u^{n-1}\right\|_{\infty}\left(1+T+T^{2}\left\|u^{n-1}\right\|_{\infty}\right),
$$

for $C, \tilde{C}>0$ only depend on the initial condition $\left(u^{0},\left(X_{v}^{0}\right)_{v \in \mathcal{V}}\right)$ and the parameters of the system. We now claim that by induction, we have for all $0<t \leq T$,

$$
\begin{gathered}
0 \leq S_{v}^{n}(t)+I_{v}^{n}(t)+R_{v}^{n}(t) \leq \hat{C} \sum_{p=0}^{a_{n}} T^{p}, \\
\left|\frac{\mathrm{d} S_{v}^{n}(t)}{\mathrm{d} t}\right|,\left|\frac{\mathrm{d} I_{v}^{n}(t)}{\mathrm{d} t}\right|,\left|\frac{\mathrm{d} R_{v}^{n}(t)}{\mathrm{d} t}\right| \leq \hat{C} \sum_{p=0}^{2 a_{n}} T^{p},
\end{gathered}
$$




$$
\left\|u^{n}\right\|_{\infty} \leq \hat{C} \sum_{p=0}^{2 a_{n}} T^{p+1}
$$

with $a_{n}=2+2 a_{n-1}$ for $n \geq 2$ and $a_{1}=1$ for some $\hat{C}>0$ only depending on $\left(u^{0},\left(X_{v}^{0}\right)_{v \in \mathcal{V}}\right)$. As $0<T<1$, we get that

$$
0 \leq S_{v}^{n}(t)+I_{v}^{n}(t)+R_{v}^{n}(t) \leq \hat{C}_{T}, \quad 0<t \leq T
$$

together with

$$
\left\|u^{n}\right\|_{\infty},\left|\frac{\mathrm{d} S_{v}^{n}(t)}{\mathrm{d} t}\right|,\left|\frac{\mathrm{d} I_{v}^{n}(t)}{\mathrm{d} t}\right|,\left|\frac{\mathrm{d} R_{v}^{n}(t)}{\mathrm{d} t}\right| \leq \hat{C}_{T},
$$

for some constant $\hat{C}_{T}>0$ depending on $\left(T, u^{0},\left(X_{v}^{0}\right)_{v \in \mathcal{V}}\right)$.

Step \#3: existence of a solution. Parabolic Schauder estimates give that the time derivative and the space derivatives up to order 2 of $u^{n}$ are uniformly Hölder continuous in compact sets. As a consequence, we can use the Arzela-Ascoli theorem to show that $\left(u^{n},\left(X_{v}^{n}\right)_{v \in \mathcal{V}}\right)$ converges (up to sequences) toward $\left(u,\left(X_{v}\right)_{v \in \mathcal{V}}\right)$ in $\mathcal{C}_{l o c}^{1,2}((0, T) \times$ $\mathcal{G}) \times \mathcal{C}_{\text {loc }}^{1}((0, T)) \times \mathcal{C}_{\text {loc }}^{1}((0, T)) \times \mathcal{C}_{\text {loc }}^{1}((0, T))$. Passing to the limit $n \rightarrow+\infty$ in (3.1)-(3.2)-(3.3) we get that $\left(u,\left(X_{v}\right)_{v \in \mathcal{V})}\right.$ satisfies (2.1)-(2.2) subject to boundary conditions (2.3).

As a by product of the proof we get that for the just constructed solution $\left(u,\left(X_{v}\right)_{v \in \mathcal{V}}\right)$ we have the uniform bounds:

$$
0<S_{v}(t) \leq S_{v}^{0}, \quad 0<t \leq T, \quad v \in \mathcal{V}
$$

and

$$
0 \leq I_{v}(t), R_{v}(t) \quad 0<t \leq T
$$

together with

$$
0 \leq u_{e}(t, x), \quad 0<t \leq T, \quad x \in \Omega_{e}, \quad e \in \mathcal{E} .
$$

The fact that $I_{v}(t) \geq 0$ implies thanks to the strong maximum principle that in fact

$$
0<u_{e}(t, x), \quad 0<t \leq T, \quad x \in \Omega_{e}, \quad e \in \mathcal{E},
$$

which in turn gives that $I_{v}(t)>0$ for each $v \in \mathcal{V}$ since

$$
\begin{aligned}
I_{v}^{n}(t)= & I_{v}^{0} e^{-\left(\eta_{v}+\bar{\lambda}_{v}\right) t+\int_{0}^{t} S_{v}^{n}(s) \mathrm{d} s} \\
& +\sum_{e \sim v} \alpha_{e}^{v} \int_{0}^{t} e^{-\left(\eta_{v}+\bar{\lambda}_{v}\right)(t-s)+\int_{s}^{t} S_{v}^{n}(\tau) \mathrm{d} \tau} u_{e}^{n-1}(s, v) \mathrm{d} s>0 .
\end{aligned}
$$


Finally, we use the conservation of mass which tells us that

$$
\sum_{e \in \mathcal{E}} \int_{\Omega_{e}} u_{e}(t, x) \mathrm{d} x+\sum_{v \in \mathcal{V}}\left(S_{v}(t)+I_{v}(t)+R_{v}(t)\right)=M^{0}>0, \quad 0<t \leq T
$$

such that both $I_{v}(t)$ and $R_{v}(t)$ are uniformly bounded in time, together with their derivatives. This also implies that there exists a constant $M>0$, depending only $\left(u^{0},\left(X_{v}^{0}\right)_{v \in \mathcal{V}}\right)$ such that

$$
0<u_{e}(t, x) \leq M, \quad 0<t \leq M, \quad x \in \Omega_{e}
$$

Using again parabolic regularity, we obtain the solution $\left(u,\left(X_{v}\right)_{v \in \mathcal{V}}\right)$ is global in time and satisfies (2.1)-(2.2)-(2.3) in the classical sense.

\subsection{Uniqueness}

Let assume that $\left(u,\left(X_{v}\right)_{v \in \mathcal{V}}\right)$ and $\left(\tilde{u},\left(\tilde{X}_{v}\right)_{v \in \mathcal{V}}\right)$ are two classical solutions to (2.2)-(2.3)-(2.1) starting from the same initial datum $\left(u^{0},\left(X_{v}^{0}\right)_{v \in \mathcal{V}}\right)$. We denote $\left(\hat{u},\left(\hat{X}_{v}\right)_{v \in \mathcal{V}}\right)$ where for each $e \in \mathcal{E}$

$$
\hat{u}_{e}=u_{e}-\tilde{u}_{e}
$$

and each $v \in \mathcal{V}$

$$
\hat{X}_{v}=\left(\hat{S}_{v}, \hat{I}_{v}, \hat{R}_{v}\right)=\left(S_{v}-\tilde{S}_{v}, I_{v}-\tilde{I}_{v}, R_{v}-\tilde{R}_{v}\right) .
$$

By linearity, we get that for $e \in \mathcal{E}$

$$
\partial_{t} \hat{u}_{e}=d_{e} \partial_{x}^{2} \hat{u}_{e}, \quad t>0, \quad x \in \stackrel{\circ}{\Omega}_{e},
$$

together with

$$
D_{v} \partial_{n} \hat{\mathbf{u}}_{v}(t)+K_{v} \hat{\mathbf{u}}_{v}(t)=\Lambda_{v} \hat{\mathbf{I}}_{v}(t), \quad t>0, \quad v \in \mathcal{V}
$$

On the other, one computes that $\hat{X}_{v}$ satisfies for each $v \in \mathcal{V}$

$$
\left\{\begin{array}{l}
\hat{S}_{v}^{\prime}(t)=-\tau_{v}\left(S_{v}(t) \hat{I}_{v}(t)+\hat{S}_{v}(t) \tilde{I}_{v}(t)\right), \\
\hat{I}_{v}^{\prime}(t)=\tau_{v}\left(S_{v}(t) \hat{I}_{v}(t)+\hat{S}_{v}(t) \tilde{I}_{v}(t)\right)-\eta_{v} \hat{I}_{v}(t)+\sum_{e \sim v} \alpha_{e}^{v} \hat{u}_{e}(t, v)-\bar{\lambda}_{v} \hat{I}_{v}(t), \\
\hat{R}_{v}^{\prime}(t)=\eta_{v} \hat{I}_{v}(t),
\end{array}\right.
$$


We define the energy

$$
\mathscr{E}(t):=\frac{1}{2} \sum_{e \in \mathcal{E}} \int_{\Omega_{e}}\left(\hat{u}_{e}(t, x)\right)^{2} \mathrm{~d} x+\frac{1}{2} \sum_{v \in \mathcal{V}}\left(\hat{S}_{v}(t)^{2}+\hat{I}_{v}(t)^{2}+\hat{R}_{v}(t)^{2}\right),
$$

and note that $\mathscr{E}(0)=0$ by definition. Next, differentiating $\mathscr{E}(t)$, we obtain

$$
\begin{aligned}
\mathscr{E}^{\prime}(t)= & \sum_{e \in \mathcal{E}} \int_{\Omega_{e}} \hat{u}_{e}(t, x) \partial_{t} \hat{u}_{e}(t, x) \mathrm{d} x \\
& +\sum_{v \in \mathcal{V}}\left(\hat{S}_{v}(t) \hat{S}_{v}^{\prime}(t)+\hat{I}_{v}(t) \hat{I}_{v}^{\prime}(t)+\hat{R}_{v}(t) \hat{R}_{v}^{\prime}(t)\right) \\
:= & \mathscr{E}_{u}(t)+\mathscr{E}_{X}(t) .
\end{aligned}
$$

On the one hand, we have

$$
\begin{aligned}
\mathscr{E}_{u}(t) & =\sum_{e \in \mathcal{E}} \int_{\Omega_{e}} \hat{u}_{e}(t, x) \partial_{t} \hat{u}_{e}(t, x) \mathrm{d} x=\sum_{e \in \mathcal{E}} d_{e} \int_{\Omega_{e}} \hat{u}_{e}(t, x) \partial_{x}^{2} \hat{u}_{e}(t, x) \mathrm{d} x \\
& =-\sum_{e \in \mathcal{E}} d_{e} \int_{\Omega_{e}}\left(\partial_{x} \hat{u}_{e}(t, x)\right)^{2} \mathrm{~d} x+\sum_{e \in \mathcal{E}} d_{e}\left[\hat{u}_{e}(t, x) \partial_{t} \hat{u}_{e}(t, x)\right]_{\partial \Omega_{e}} \\
& \leq \sum_{v \in \mathcal{V}}\left\langle D_{v} \partial_{n} \hat{\mathbf{u}}_{v}(t), \hat{\mathbf{u}}_{v}(t)\right\rangle \\
& =\sum_{v \in \mathcal{V}}\left\langle\Lambda_{v} \hat{\mathbf{I}}_{v}(t)-K_{v} \hat{\mathbf{u}}_{v}(t), \hat{\mathbf{u}}_{v}(t)\right\rangle \\
& =\sum_{v \in \mathcal{V}}\left\langle\Lambda_{v} \hat{\mathbf{I}}_{v}(t)-A_{v} \hat{\mathbf{u}}_{v}(t), \hat{\mathbf{u}}_{v}(t)\right\rangle-\sum_{v \in \mathcal{V}}\left\langle N_{v} \hat{\mathbf{u}}_{v}(t), \hat{\mathbf{u}}_{v}(t)\right\rangle \\
& \leq \sum_{v \in \mathcal{V}}\left\langle\Lambda_{v} \hat{\mathbf{I}}_{v}(t)-A_{v} \hat{\mathbf{u}}_{v}(t), \hat{\mathbf{u}}_{v}(t)\right\rangle,
\end{aligned}
$$

as $N_{v}$ is symmetric positive. On the other hand, we compute

$$
\begin{aligned}
\mathscr{E}_{u}(t)= & \sum_{v \in \mathcal{V}}\left(\hat{S}_{v}(t) \hat{S}_{v}^{\prime}(t)+\hat{I}_{v}(t) \hat{I}_{v}^{\prime}(t)+\hat{R}_{v}(t) \hat{R}_{v}^{\prime}(t)\right) \\
= & \sum_{v \in \mathcal{V}} \tau_{v}\left(S_{v}(t) \hat{I}_{v}(t) \hat{S}_{v}(t)+\hat{S}_{v}(t)^{2} \tilde{I}_{v}(t)+S_{v}(t) \hat{I}_{v}(t)^{2}+\hat{S}_{v}(t) \tilde{I}_{v}(t) \hat{I}_{v}(t)\right) \\
& -\sum_{v \in \mathcal{V}} \eta_{v} \hat{I}_{v}(t)^{2}-\sum_{v \in \mathcal{V}} \bar{\lambda}_{v} \hat{I}_{v}(t)^{2}+\sum_{v \in \mathcal{V}} \hat{I}_{v}(t) \sum_{e \sim v} \alpha_{e}^{v} \hat{u}_{e}(t, v) \\
& +\sum_{v \in \mathcal{V}} \eta_{v} \hat{R}_{v}(t) \hat{I}_{v}(t) \\
\leq & C \mathscr{E}(t)+\sum_{v \in \mathcal{V}} \hat{I}_{v}(t) \sum_{e \sim v} \alpha_{e}^{v} \hat{u}_{e}(t, v),
\end{aligned}
$$


where $C>0$ is some large positive constant. Next, we see that

$$
\sum_{v \in \mathcal{V}} \hat{I}_{v}(t) \sum_{e \sim v} \alpha_{e}^{v} \hat{u}_{e}(t, v)=\sum_{v \in \mathcal{V}}\left\langle\hat{\mathbf{I}}_{v}(t), A_{v} \hat{\mathbf{u}}_{v}(t)\right\rangle
$$

such that we obtain

$$
\mathscr{E}^{\prime}(t) \leq C \mathscr{E}(t)+\sum_{v \in \mathcal{V}}\left\langle\Lambda_{v} \hat{\mathbf{I}}_{v}(t)-A_{v} \hat{\mathbf{u}}_{v}(t), \hat{\mathbf{u}}_{v}(t)\right\rangle+\sum_{v \in \mathcal{V}}\left\langle\hat{\mathbf{I}}_{v}(t), A_{v} \hat{\mathbf{u}}_{v}(t)\right\rangle
$$

Next, if we denote $\hat{\mathbf{w}}_{v}(t):=\frac{1}{2} A_{v}^{-1 / 2}\left(\Lambda_{v}+A_{v}\right) \hat{\mathbf{I}}_{v}(t)-A_{v}^{1 / 2} \hat{\mathbf{u}}_{v}(t)$, we compute

$$
\begin{aligned}
0 \leq \sum_{v \in \mathcal{V}}\left\langle\hat{\mathbf{w}}_{v}(t), \hat{\mathbf{w}}_{v}(t)\right\rangle= & \frac{1}{4} \sum_{v \in \mathcal{V}}\left\langle A_{v}^{-1}\left(\Lambda_{v}+A_{v}\right)^{2} \hat{\mathbf{I}}_{v}(t), \hat{\mathbf{I}}_{v}(t)\right\rangle \\
& -\sum_{v \in \mathcal{V}}\left\langle\left(\Lambda_{v}+A_{v}\right) \hat{\mathbf{I}}_{v}(t), \hat{\mathbf{u}}_{v}(t)\right\rangle \\
& +\sum_{v \in \mathcal{V}}\left\langle A_{v} \hat{\mathbf{u}}_{v}(t), \hat{\mathbf{u}}_{v}(t)\right\rangle \\
= & \frac{1}{4} \sum_{v \in \mathcal{V}}\left\langle A_{v}^{-1}\left(\Lambda_{v}+A_{v}\right)^{2} \hat{\mathbf{I}}_{v}(t), \hat{\mathbf{I}}_{v}(t)\right\rangle \\
& -\sum_{v \in \mathcal{V}}\left\langle\Lambda_{v} \hat{\mathbf{I}}_{v}(t)-A_{v} \hat{\mathbf{u}}_{v}(t), \hat{\mathbf{u}}_{v}(t)\right\rangle \\
& -\sum_{v \in \mathcal{V}}\left\langle\hat{\mathbf{I}}_{v}(t), A_{v} \hat{\mathbf{u}}_{v}(t)\right\rangle .
\end{aligned}
$$

As a consequence, we get

$$
\begin{aligned}
\mathscr{E}^{\prime}(t) & \leq C \mathscr{E}(t)+\frac{1}{4} \sum_{v \in \mathcal{V}}\left\langle A_{v}^{-1}\left(\Lambda_{v}+A_{v}\right)^{2} \hat{\mathbf{I}}_{v}(t), \hat{\mathbf{I}}_{v}(t)\right\rangle-\sum_{v \in \mathcal{V}}\left\langle\hat{\mathbf{w}}_{v}(t), \hat{\mathbf{w}}_{v}(t)\right\rangle \\
& \leq \tilde{C} \mathscr{E}(t),
\end{aligned}
$$

for some $\tilde{C}>0$ and we conclude that $\mathscr{E}(t)=0$ for all time which then implies that $\hat{u}=0$ and $\hat{X}_{v}=0$.

\section{Long-time behavior of the solutions}

Throughout this section, we denote by $\left(u,\left(X_{v}\right)_{v \in \mathcal{V}}\right)$ the unique positive bounded classical solution of the Cauchy problem (2.1)-(2.2)-(2.3) as given by Theorem 1 and which further satisfies the conservation of total population, namely

$$
\sum_{e \in \mathcal{E}} \int_{\Omega_{e}} u_{e}(t, x) \mathrm{d} x+\sum_{v \in \mathcal{V}}\left(S_{v}(t)+I_{v}(t)+R_{v}(t)\right)=M^{0}>0, \quad \forall t>0
$$




\subsection{Final total populations: general results}

As $0<S_{v}(t)<S_{v}^{0}$ and $S_{v}(t)$ is strictly decreasing, it asymptotically converges towards a limit that we denote

$$
S_{v}^{\infty}:=\lim _{t \rightarrow+\infty} S_{v}(t), \quad v \in \mathcal{V}
$$

Furthermore, as $R_{v}(t)$ is strictly increasing and uniformly bounded, it asymptotically converges towards a limit that is denoted

$$
0<R_{v}^{\infty}:=\lim _{t \rightarrow+\infty} R_{v}(t)<\infty, \quad v \in \mathcal{V}
$$

But as for each $t>0$

$$
R_{v}(t)=\eta_{v} \int_{0}^{t} I_{v}(s) \mathrm{d} s
$$

this implies that

$$
\mathcal{I}_{v}(t):=\int_{0}^{t} I_{v}(s) \mathrm{d} s \longrightarrow \mathcal{I}_{v}^{\infty}=\frac{R_{v}^{\infty}}{\eta_{v}}<\infty \text { as } t \rightarrow+\infty
$$

which in turn proves that

$$
I_{v}^{\infty}=\lim _{t \rightarrow+\infty} I_{v}(t)=0
$$

pending that we prove that the limit of $I_{v}(t)$ as $t \rightarrow+\infty$ exists. To see that, we use the fact that

$$
\frac{\mathrm{d} I_{v}(t)}{\mathrm{d} t}+\frac{\mathrm{d} S_{v}(t)}{\mathrm{d} t}-\frac{\eta_{v}+\bar{\lambda}_{v}}{\tau_{v}} \frac{\mathrm{d} \ln S_{v}(t)}{\mathrm{d} t}=\sum_{e \sim v} \alpha_{e}^{v} u_{e}(t, v), \quad v \in \mathcal{V},
$$

to obtain that

$$
\begin{aligned}
& I_{v}(t)+S_{v}(t)-\frac{\eta_{v}+\bar{\lambda}_{v}}{\tau_{v}} \ln S_{v}(t)-I_{v}^{0}-S_{v}^{0}+\frac{\eta_{v}+\bar{\lambda}_{v}}{\tau_{v}} \ln S_{v}^{0} \\
& \quad=\sum_{e \sim v} \alpha_{e}^{v} \int_{0}^{t} u_{e}(s, v) \mathrm{d} s, \quad v \in \mathcal{V} .
\end{aligned}
$$

We now argue that the left-hand side of the previous equation is uniformly bounded as $S_{v}^{\infty}=S_{v}^{0} e^{-\tau_{v} \mathcal{I}_{v}^{\infty}}>0$, which ensures that $\int_{0}^{t} u_{e}(s, v) \mathrm{d} s$ has a limit as $t \rightarrow+\infty$ by positivity of $u_{e}$. This in turn implies that the limit of $I_{v}(t)$ as $t \rightarrow+\infty$ exists. 
If one recall the notation $m(t)$ for the total population on the edges then we have

$$
m(t)=\sum_{e \in \mathcal{E}} \int_{\Omega_{e}} u_{e}(t, x) \mathrm{d} x
$$

and it verifies

$$
\sum_{v \in \mathcal{V}}\left(S_{v}(t)+I_{v}(t)+R_{v}(t)\right)+m(t)=\sum_{v \in \mathcal{V}}\left(S_{v}^{0}+I_{v}^{0}\right)+\sum_{e \in \mathcal{E}} \int_{\Omega_{e}} u_{e}^{0}(x) \mathrm{d} x
$$

The above computations shows that $m(t)$ has a limit as $t \longrightarrow+\infty$, that we denote $m_{\infty}$ and which satisfies

$$
\sum_{v \in \mathcal{V}}\left(S_{v}^{\infty}+R_{v}^{\infty}\right)+m_{\infty}=\sum_{v \in \mathcal{V}}\left(S_{v}^{0}+I_{v}^{0}\right)+\sum_{e \in \mathcal{E}} \int_{\Omega_{e}} u_{e}^{0}(x) \mathrm{d} x
$$

We shall also keep in mind that

$$
S_{v}^{\infty}=S_{v}^{0} e^{-\tau_{v} \mathcal{I}_{v}^{\infty}}=S_{v}^{0} e^{-\frac{\tau_{v}}{\eta_{v}} R_{v}^{\infty}}, \text { or } R_{v}^{\infty}=-\frac{\eta_{v}}{\tau_{v}} \ln \frac{S_{v}^{\infty}}{S_{v}^{0}}, \quad v \in \mathcal{V}
$$

And so if we introduce the function $\Psi_{v}(x):=x-\frac{\eta_{v}}{\tau_{v}} \ln x$, then the above conservation of mass can be written as

$$
\sum_{v \in \mathcal{V}} \Psi_{v}\left(S_{v}^{\infty}\right)+m_{\infty}=\sum_{v \in \mathcal{V}}\left(I_{v}^{0}+\Psi_{v}\left(S_{v}^{0}\right)\right)+\sum_{e \in \mathcal{E}} \int_{\Omega_{e}} u_{e}^{0}(x) \mathrm{d} x
$$

On the other, one can compute that

$$
\frac{\mathrm{d} m(t)}{\mathrm{d} t}=\sum_{v \in \mathcal{V}} \bar{\lambda}_{v} I_{v}(t)-\sum_{v \in \mathcal{V}}\left\langle A_{v} \mathbf{u}_{v}(t), \mathbf{1}_{\delta_{v}}\right\rangle
$$

such that

$$
m(t)+\sum_{v \in \mathcal{V}} \int_{0}^{t}\left\langle A_{v} \mathbf{u}_{v}(s), \mathbf{1}_{\delta_{v}}\right\rangle \mathrm{d} s=\sum_{e \in \mathcal{E}} \int_{\Omega_{e}} u_{e}^{0}(x) \mathrm{d} x+\sum_{v \in \mathcal{V}} \bar{\lambda}_{v} \mathcal{I}_{v}(t)
$$

Now, as $m(t)$ and each $\mathcal{I}_{v}(t)$ are convergent we deduce that all $\int_{0}^{t} \mathbf{u}_{v}(s) \mathrm{d} s$ are also convergent so that

$$
m_{\infty}+\sum_{v \in \mathcal{V}} \int_{0}^{\infty}\left\langle A_{v} \mathbf{u}_{v}(s), \mathbf{1}_{\delta_{v}}\right\rangle \mathrm{d} s=\sum_{e \in \mathcal{E}} \int_{\Omega_{e}} u_{e}^{0}(x) \mathrm{d} x+\sum_{v \in \mathcal{V}} \bar{\lambda}_{v} \mathcal{I}_{v}^{\infty}
$$


and

$$
\int_{0}^{\infty} \mathbf{u}_{v}(s) \mathrm{d} s<\infty, \quad v \in \mathcal{V}
$$

which proves that

$$
\mathbf{u}_{v}(t) \longrightarrow 0 \text { as } t \rightarrow+\infty, \quad v \in \mathcal{V}
$$

And the boundary conditions imply that

$$
\partial_{n} \mathbf{u}_{v}(t) \longrightarrow 0 \text { as } t \rightarrow+\infty, \quad v \in \mathcal{V}
$$

Next, we define the sequence of functions $u_{e}^{n}(t, x)=u_{e}(t+n, x)$ for each $e \in \mathcal{E}$ and $X_{v}^{n}(t)=X_{v}(t+n)$ for each $v \in \mathcal{V}$ which are uniformly bounded such that one can extract a convergent subsequence. On the one hand we have that $\lim _{n \rightarrow \infty} X_{v}^{n}(t)=X_{v}^{\infty}=$ $\left(S_{v}^{\infty}, 0, R_{v}^{\infty}\right)$ and on the other if $u_{e}^{\infty}(t, x)=\lim _{n \rightarrow \infty} u_{e}^{n}(t, x)$ it is solution of

$$
\partial_{t} u_{e}^{\infty}(t, x)=d_{e} \partial_{x}^{2} u_{e}^{\infty}(t, x)
$$

with the boundary conditions

$$
\partial_{n} \mathbf{u}_{v}^{\infty}(t)=\mathbf{u}_{v}^{\infty}(t)=\mathbf{0}_{\delta_{v}}, \quad v \in \mathcal{V}
$$

This then shows that $u_{e}^{\infty}(t, x)=0, t>0$ and $x \in \stackrel{\circ}{\Omega}_{e}$ for each $e \in \mathcal{E}$. As there is unicity of the limit, we deduce that

$$
\lim _{t \rightarrow+\infty} u_{e}(t, x)=0, \quad e \in \mathcal{E}
$$

From which we also get that $m_{\infty}=0$ and that

$$
\sum_{v \in \mathcal{V}} \Psi_{v}\left(S_{v}^{\infty}\right)=\sum_{v \in \mathcal{V}}\left(I_{v}^{0}+\Psi_{v}\left(S_{v}^{0}\right)\right)+\sum_{e \in \mathcal{E}} \int_{\Omega_{e}} u_{e}^{0}(x) \mathrm{d} x
$$

We also get from (4.3), that

$$
\sum_{v \in \mathcal{V}} \int_{0}^{\infty}\left\langle A_{v} \mathbf{u}_{v}(s), \mathbf{1}_{\delta_{v}}\right\rangle \mathrm{d} s=\sum_{e \in \mathcal{E}} \int_{\Omega_{e}} u_{e}^{0}(x) \mathrm{d} x+\sum_{v \in \mathcal{V}} \bar{\lambda}_{v} \mathcal{I}_{v}^{\infty}
$$

Finally, as a consequence of (4.1), the final total populations of infected individuals at each vertices satisfy the following scalar differential equation

$$
\frac{\mathrm{d} \mathcal{I}_{v}(t)}{\mathrm{d} t}=S_{v}^{0}\left(1-e^{-\tau_{v} \mathcal{I}_{v}(t)}\right)-\eta_{v} \mathcal{I}_{v}(t)+I_{v}^{0}
$$




$$
+\sum_{e \sim v} \alpha_{e}^{v} \int_{0}^{t} u_{e}(s, v) \mathrm{d} s-\bar{\lambda}_{v} \mathcal{I}_{v}(t), \quad v \in \mathcal{V}
$$

Passing to the limit as $t \rightarrow+\infty$, we get

$$
0=S_{v}^{0}\left(1-e^{-\tau_{v} \mathcal{I}_{v}^{\infty}}\right)-\eta_{v} \mathcal{I}_{v}^{\infty}+I_{v}^{0}+\sum_{e \sim v} \alpha_{e}^{v} \int_{0}^{\infty} u_{e}(s, v) \mathrm{d} s-\bar{\lambda}_{v} \mathcal{I}_{v}^{\infty}, \quad v \in \mathcal{V}
$$

To summarize, we have proved the following result.

Theorem 2 For each $\left(S_{v}^{0}, I_{v}^{0}\right) \geq \mathbf{0}$ with $S_{v}^{0}>0, \sum_{v \in \mathcal{V}} I_{v}^{0}>0$ and $u^{0} \in B C\left(\mathcal{G}, \mathbb{R}^{+}\right)$ that satisfy the boundary condition (2.3), the long time behavior of the unique corresponding solution $\left(u,\left(X_{v}\right)_{v \in \mathcal{V}}\right)$ is given by

$\lim _{t \rightarrow+\infty} u_{e}(t, x)=0, \quad x \in \Omega_{e}, \quad e \in \mathcal{E}, \quad$ with $\quad \int_{0}^{\infty} u_{e}(s, v) \mathrm{d} s<+\infty, \quad(v, e) \in \mathcal{G}$, and

$$
\lim _{t \rightarrow+\infty}\left(S_{v}(t), I_{v}(t), R_{v}(t)\right)=\left(S_{v}^{0} e^{-\tau_{v} \mathcal{I}_{v}^{\infty}}, 0, \eta_{v} \mathcal{I}_{v}^{\infty}\right), \quad v \in \mathcal{V}
$$

where the final total populations of infected individuals $0<\mathcal{I}_{v}^{\infty}<\infty$ at each vertices $v \in \mathcal{V}$ are solutions of the system

$$
\left\{\begin{aligned}
S_{v}^{0} e^{-\tau_{v} \mathcal{I}_{v}^{\infty}}+\eta_{v} \mathcal{I}_{v}^{\infty} & =I_{v}^{0}+S_{v}^{0}+\sum_{e \sim v} \alpha_{e}^{v} \int_{0}^{\infty} u_{e}(s, v) \mathrm{d} s-\bar{\lambda}_{v} \mathcal{I}_{v}^{\infty}, \quad v \in \mathcal{V}, \\
\sum_{v \in \mathcal{V}} \int_{0}^{\infty}\left\langle A_{v} \mathbf{u}_{v}(s), \mathbf{1}_{\delta_{v}}\right\rangle \mathrm{d} s & =\sum_{e \in \mathcal{E}} \int_{\Omega_{e}} u_{e}^{0}(x) \mathrm{d} x+\sum_{v \in \mathcal{V}} \bar{\lambda}_{v} \mathcal{I}_{v}^{\infty} .
\end{aligned}\right.
$$

As a consequence, $\left(\mathcal{I}_{v}^{\infty}\right)_{v \in \mathcal{V}}$ belongs to the parametrized submanifold given by

$$
\sum_{v \in \mathcal{V}}\left(S_{v}^{0} e^{-\tau_{v} \mathcal{I}_{v}^{\infty}}+\eta_{v} \mathcal{I}_{v}^{\infty}\right)=M^{0}
$$

Remark 4.1 Equivalently, $\left(S_{v}^{\infty}\right)_{v \in \mathcal{V}}$ belongs to the parametrized submanifold given by

$$
\sum_{v \in \mathcal{V}}\left(S_{v}^{\infty}-\frac{\eta_{v}}{\tau_{v}} \log \left(S_{v}^{\infty}\right)+\frac{\eta_{v}}{\tau_{v}} \log \left(S_{v}^{0}\right)\right)=M^{0}
$$

and $\left(R_{v}^{\infty}\right)_{v \in \mathcal{V}}$ belongs to the parametrized submanifold given by

$$
\sum_{v \in \mathcal{V}}\left(S_{v}^{0} \exp \left(-\tau_{v} / \eta_{v} R_{v}^{\infty}\right)+R_{v}^{\infty}\right)=M^{0}
$$


The equations (4.7), (4.8), and (4.9) also read $\sum_{v \in \mathcal{V}}\left(S_{v}^{\infty}+R_{v}^{\infty}\right)=M^{0}$, which is nothing but (4.2) since we have proved that $m_{\infty}=0$.

Remark 4.2 If we assume that $\tau=\tau_{v}>0$ and $\eta=\eta_{v}>0$ are independent of $v \in \mathcal{V}$ and let $\widetilde{S_{v}}=\tau / \eta S_{v}, \widetilde{R_{v}^{\infty}}=\exp \left(-\tau / \eta R_{v}^{\infty}\right)$ and $\widetilde{\mathcal{I}_{v}^{\infty}}=\exp \left(-\tau \mathcal{I}_{v}^{\infty}\right)$. Then, equations (4.7), (4.8), and (4.9) are respectively equivalent to

$$
\begin{aligned}
& \prod_{v \in \mathcal{V}} \exp \left(\widetilde{S_{v}^{\infty}}\right) \frac{\widetilde{S_{v}^{0}}}{\widetilde{S_{v}^{\infty}}}=\exp \left(\frac{\tau}{\eta} M^{0}\right), \\
& \prod_{v \in \mathcal{V}} \frac{\exp \left(\widetilde{S_{v}^{0}} \widetilde{R_{v}^{\infty}}\right)}{\widetilde{R_{v}^{\infty}}}=\exp \left(\frac{\tau}{\eta} M^{0}\right),
\end{aligned}
$$

and

$$
\prod_{v \in \mathcal{V}} \frac{\exp \left(\widetilde{S_{v}^{0}} \widetilde{\mathcal{I}_{v}^{\infty}}\right)}{\widetilde{\mathcal{I}_{v}^{\infty}}}=\exp \left(\frac{\tau}{\eta} M^{0}\right)
$$

The common right hand side features $\frac{\tau}{\eta} M^{0}$ that is nothing but the traditional basic reproductive number $\mathscr{R}_{0}$.

Remark 4.3 The above equation (4.4) can be interpreted as the counter-part on graphs to equations that were already derived in a spatially continuous setting (Diekmann 1978; Berestycki et al. 2020). In our case, we can rewrite (4.4) as

$$
\frac{\mathrm{d} \mathcal{I}_{v}(t)}{\mathrm{d} t}=\mathcal{L}_{v} \cdot \mathcal{I}_{v}(t)+f_{v}\left(\mathcal{I}_{v}(t)\right)+I_{v}^{0}, \quad v \in \mathcal{V}
$$

where $f_{v}(x):=S_{v}^{0}\left(1-e^{-\tau_{v} x}\right)-\eta_{v} x$ and $\mathcal{L}_{v} \cdot \mathcal{I}_{v}(t):=\sum_{e \sim v} \alpha_{e}^{v} \int_{0}^{t} u_{e}(s, v) \mathrm{d} s-$ $\bar{\lambda}_{v} \mathcal{I}_{v}(t)$. As a consequence, Eq. (4.4) can be thought as a discrete reaction-diffusion equation set on the graph $\mathcal{G}$ where $\mathcal{L}_{v}$ is a heterogenous diffusion process which takes into account the connectivity of the graph, while $f_{v}$ encodes the nonlinear reaction terms. As in Diekmann (1978) and Berestycki et al. (2020), the nonlinearity has some concavity properties as

$$
x \mapsto \frac{f_{v}(x)}{x} \text { is decreasing and } f_{v}(x) \leq\left(S_{v}^{0} \tau_{v}-\eta_{v}\right) x \quad \forall(x, v) \in[0, \infty) \times \mathcal{V}
$$

We do not pursue this direction as it would require a thorough study of the heterogeneous diffusion operator $\mathcal{L}_{v}$ which is beyond the scope of the present manuscript.

\subsection{Final total populations of infected individuals: further properties}

The aim of this section is to present further qualitative results on the final total configuration $\left(\mathcal{I}_{v}^{\infty}\right)_{v \in \mathcal{V}}$ in the fully symmetric case where one can obtain closed form 
formula and in the case of two vertices where we manage to obtain sharp bounds on the final total populations of infected individuals. In each case, we manage to relate these quantities to standard basic and effective reproductive number for classical SIR model (Diekmann et al. 1990). We also refer to Stolerman et al. (2015) in the case of networks without diffusion on the edges where properties of the basic reproduction number are linked to the geometry and heterogeneity of the network.

\subsubsection{Fully symmetric case}

We assume that the length $\ell_{e}$ of every edge $e \in \mathcal{E}$ is equal to a reference length $\ell$. For every $e \in \mathcal{E}$, the diffusion coefficient $d_{e}$ is equal to $d$. We moreover suppose that for every vertex $v \in \mathcal{V}, S_{v}^{0}=S^{0}, I_{v}^{0}=I^{0}$ and $R_{v}^{0}=R^{0}$. We also assume that $\tau=\tau_{v}>0$ and $\eta=\eta_{v}>0$ are independent of $v \in \mathcal{V}$. In the same spirit, $\lambda_{e}^{v}=\lambda$ and $\alpha_{e}^{v}=\alpha$ for every $e \in \mathcal{E}$ and $v \in \mathcal{V}$. We also assume $v_{e_{i}, e_{j}}^{v}=v$ for every edges incident to the vertex $v$. Finally, the components $u_{e}^{0}$ of initial condition on each edges $e \in \mathcal{E}$ are supposed to be even with respect to the center of the interval $\Omega_{e}=[0, \ell]$. Thanks to all these assumptions, $\mathcal{I}_{v}^{\infty}$ does not depend on the vertex $v \in \mathcal{V}$ and we set $\mathcal{I}_{v}^{\infty}=\mathcal{I}^{\infty}$. Let us recall the notation $c \mathcal{V}$ for the cardinal of the set $\mathcal{V}$. The parametrized submanifold given by (4.7) becomes

$$
S^{\infty}+R^{\infty}=S^{0} e^{-\tau \mathcal{I}^{\infty}}+\eta \mathcal{I}^{\infty}=\widetilde{M^{0}}
$$

where $\widetilde{M^{0}}=M^{0} / c \mathcal{V}$. We can transform this relation as

$$
S^{0} e^{-\tau \mathcal{I}^{\infty}}+\frac{\eta}{\tau} \tau \mathcal{I}^{\infty}-\widetilde{M^{0}}=0
$$

Let $\mathscr{I}=-\tau \mathcal{I}^{\infty}$. We have to solve

$$
S^{0} e^{\mathscr{I}}-\frac{\eta}{\tau} \mathscr{I}-\widetilde{M^{0}}=0
$$

The solutions are given in terms of Lambert $\mathrm{W}$ function that is the multivalued inverse relation of the function $f(w)=w e^{w}$ for $w \in \mathbb{C}$ (Corless et al. 1996). Let us recall how to compute the real solutions of the equation $\alpha e^{x}+\beta x+\gamma=0$ for $(\alpha, \beta, \gamma) \in$ $\mathbb{R}^{*} \times \mathbb{R}^{*} \times \mathbb{R}$. Let $\Delta=\alpha / \beta \exp (-\gamma / \beta)$ be the discriminant. If $\Delta \geq 0$ or $\Delta=$ $-\exp (-1)$, the solution is unique and $x=-W_{0}(\Delta)-\gamma / \beta$ where $W_{0}$ is the principal branch. If $\Delta \in(-\exp (-1), 0)$, there are two solutions $x_{0}=-W_{0}(\Delta)-\gamma / \beta$ and $x_{-1}=-W_{-1}(\Delta)-\gamma / \beta$, where $W_{-1}$ is another branch. When $\Delta<-\exp (-1)$, there is no solution.

In our symmetric case, the discriminant writes

$$
\Delta=-\frac{S^{0} \tau}{\eta} \exp \left(-\frac{\widetilde{M^{0}} \tau}{\eta}\right)
$$


Since $\Delta<0$, there exist solutions to (4.10) if $\Delta \geq-\exp (-1)$, which is equivalent to

$$
\exp \left(\frac{\widetilde{M^{0}} \tau}{\eta}-1\right) \geq \frac{S^{0} \tau}{\eta}
$$

We recall that when we consider the standard SIR model (meaning in the context of this paper that we consider an isolated vertex), we can define the effective reproductive number $\mathscr{R}_{e}$ and the basic reproductive number $\mathscr{R}_{0}$ respectively given by

$$
\mathscr{R}_{e}:=\frac{S^{0} \tau}{\eta}, \quad \text { and } \quad \mathscr{R}_{0}:=\frac{M^{0} \tau}{\eta}
$$

see Diekmann et al. (1990), Van den Driessche and Watmough (2002) and Hethcote (2000) for further properties of effective and basic reproductive numbers. If we denote $\widetilde{\mathscr{R}}_{0}=\widetilde{M^{0}} \tau / \eta$, the equation (4.11) reads

$$
\exp \left(\widetilde{\mathscr{R}}_{0}-1\right) \geq \mathscr{R}_{e}
$$

This inequality is satisfied as long as $S^{0} \leq \widetilde{M^{0}}$, which is always true since $M^{0}=$ $\sum_{e \in \mathcal{E}} \int_{\Omega_{e}} u_{e}^{0}(x) \mathrm{d} x+c \mathcal{V}\left(S^{0}+I^{0}\right) \geq c \mathcal{V} S^{0}$. Since $\Delta=-\mathscr{R}_{e} \exp \left(-\widetilde{\mathscr{R}}_{0}\right)$, the solutions are

$$
\mathscr{I}_{0,-1}=-W_{0,-1}\left(-\mathscr{R}_{e} \exp \left(-\widetilde{\mathscr{R}}_{0}\right)\right)-\widetilde{\mathscr{R}}_{0},
$$

and so

$$
\mathcal{I}_{0,-1}^{\infty}=W_{0,-1}\left(-\mathscr{R}_{e} \exp \left(-\widetilde{\mathscr{R}}_{0}\right)\right) / \tau+\widetilde{\mathscr{R}}_{0} / \tau
$$

Both $W_{0,-1}\left(-\mathscr{R}_{e} \exp \left(-\widetilde{\mathscr{R}}_{0}\right)\right)<0$. However, we can show that $\mathcal{I}_{0}^{\infty}>0$ and $\mathcal{I}_{-1}^{\infty}<0$. Thus, the only possibility is

$$
\mathcal{I}^{\infty}=W_{0}\left(-\mathscr{R}_{e} \exp \left(-\widetilde{\mathscr{R}}_{0}\right)\right) / \tau+\widetilde{\mathscr{R}}_{0} / \tau
$$

We also have access to $S^{\infty}$ and $R^{\infty}$ thanks to (4.5). Since $\exp \left(-W_{0}(x)\right)=W_{0}(x) / x$, we obtain

$$
S^{\infty}=-\frac{\eta}{\tau} W_{0}\left(-\mathscr{R}_{e} \exp \left(-\widetilde{\mathscr{R}}_{0}\right)\right)
$$

and

$$
R^{\infty}=\frac{\eta}{\tau} W_{0}\left(-\mathscr{R}_{e} \exp \left(-\widetilde{\mathscr{R}}_{0}\right)\right)+\widetilde{M^{0}}
$$

We can summarize these results in the following lemma. 

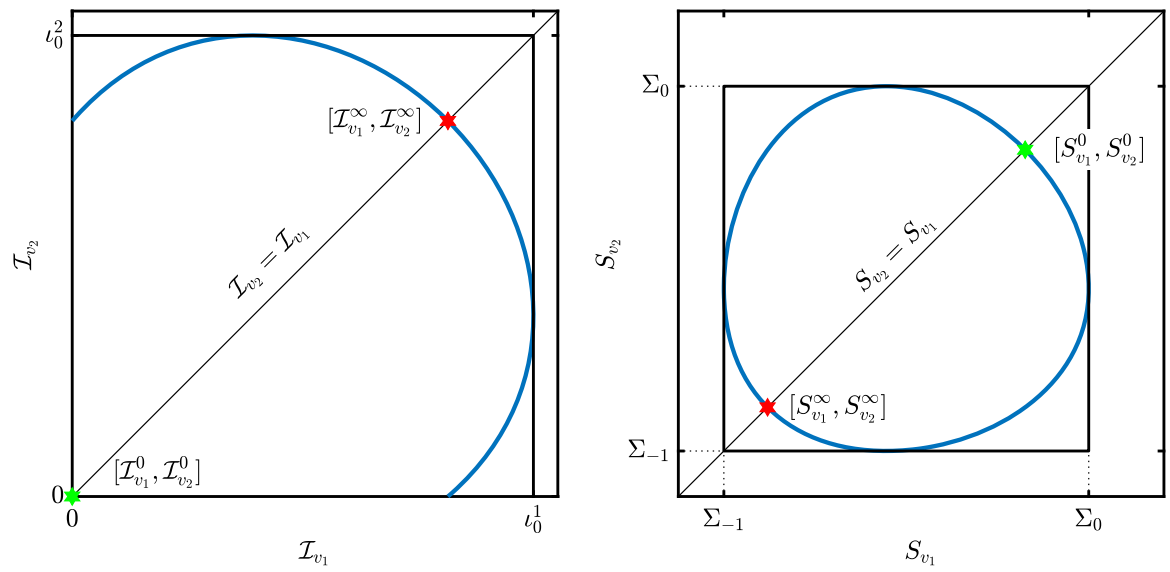

Fig. 3 Schematic visualisation (red star) of $\mathcal{I}^{\infty}=\mathcal{I}_{v_{1}}^{\infty}=\mathcal{I}_{v_{2}}^{\infty}$, resp. $S^{\infty}=S_{v_{1}}^{\infty}=S_{v_{2}}^{\infty}$, in the $\left(\mathcal{I}_{v_{1}}, \mathcal{I}_{v_{2}}\right.$ ) plane, resp. in the $\left(S_{v_{1}}, S_{v_{2}}\right)$-plane, in the fully symmetric case. The asymptotic value $\mathcal{I}^{\infty}$, resp. $S^{\infty}$, lies at the intersection of the diagonal $\mathcal{I}_{v_{1}}=\mathcal{I}_{v_{2}}$, resp. $S_{v_{1}}=S_{v_{2}}$, and the implicit curve given by (4.7), resp. (4.8) (color figure online)

Lemma 4.4 (Fully symmetric case.) Assume that our model is fully symmetric, then the final total population of infected individuals as given by Theorem 2 is independent on the vertex that is $\mathcal{I}_{v}^{\infty}=\mathcal{I}^{\infty}$ for each $v \in \mathcal{V}$, and $\mathcal{I}^{\infty}$ has the following closed form formula

$$
\mathcal{I}^{\infty}=\frac{W_{0}\left(-\mathscr{R}_{e} \exp \left(-\mathscr{R}_{0} / c \mathcal{V}\right)\right)}{\tau}+\frac{\mathscr{R}_{0}}{c_{\mathcal{V}} \tau}
$$

where $\mathscr{R}_{e}$ and $\mathscr{R}_{0}$ are respectively the effective and basic reproductive number defined in (4.12) and $c_{\mathcal{V}}$ the cardinal of $\mathcal{V}$. See Fig. 3 for an illustration.

\subsubsection{Case of two vertices}

In this simple case, it is possible to build explicit formulas to deal with the implicit submanifold equations (4.7), (4.8), and (4.9). Let $\mathscr{R}_{0, v_{k}}:=M^{0} \tau_{v_{k}} / \eta_{v_{k}}$ and $\mathscr{R}_{e, v_{k}}:=$ $S_{v_{k}}^{0} \tau_{v_{k}} / \eta_{v_{k}}, k=1,2$ be respectively the local to vertex $v_{k}$ basic and effective reproductive number. Then,

$$
S_{v_{1}}^{\infty}=-\frac{\eta_{v_{1}}}{\tau_{v_{1}}} W\left(-\exp \left(-\mathscr{R}_{0, v_{1}}\right) \mathscr{R}_{e, v_{1}}\left(\mathscr{R}_{e, v_{2}}\right)^{\frac{\tau_{v_{1}} \eta_{v_{2}}}{\tau_{v_{2}} \eta_{v_{1}}}} \frac{\exp \left(S_{v_{2}}^{\infty} \tau_{v_{1}} / \eta_{v_{1}}\right)}{\left(S_{v_{2}}^{\infty} \tau_{v_{2}} / \eta_{v_{2}}\right)^{\frac{\tau_{v_{1}} \eta_{v_{2}}}{\tau_{v_{2}} \eta_{v_{1}}}}}\right),
$$


where the Lambert $\mathrm{W}$ function $W$ can be either $W_{0}$ or $W_{-1}$. Indeed, the argument of $W$ being negative, two solutions have to be considered. We obviously also have

$$
S_{v_{2}}^{\infty}=-\frac{\eta_{v_{2}}}{\tau_{v_{2}}} W\left(-\exp \left(-\mathscr{R}_{0, v_{2}}\right) \mathscr{R}_{e, v_{2}}\left(\mathscr{R}_{e, v_{1}}\right)^{\frac{\tau_{v_{2}} \eta_{v_{1}}}{\tau_{v_{1}} \eta_{v_{2}}}} \frac{\exp \left(S_{v_{1}}^{\infty} \tau_{v_{2}} / \eta_{v_{2}}\right)}{\left(S_{v_{1}}^{\infty} \tau_{v_{1}} / \eta_{v_{1}}\right)^{\frac{\tau_{v_{2}} \eta_{v_{1}}}{\tau_{v_{1}} \eta_{v_{2}}}}}\right),
$$

Due to the definition of the domain of the Lambert $\mathrm{W}$ function, the argument has to be greater than $-\exp (-1)$. So, the following inequality must be satisfied for $S_{v_{2}}^{\infty}$ (respectively of $S_{v_{1}}^{\infty}$ )

$$
\frac{-\exp \left(-\mathscr{R}_{0, v_{1}}\right) \mathscr{R}_{e, v_{1}}\left(\mathscr{R}_{e, v_{2}}\right)^{\frac{\tau_{v_{1}} \eta_{v_{2}}}{\tau_{v_{2}} \eta_{v_{1}}}}}{\left(S_{v_{2}}^{\infty} \tau_{v_{2}} / \eta_{v_{2}}\right)^{\frac{\tau_{v_{1}} \eta_{v_{2}}}{\tau_{v_{2}} \eta_{v_{1}}}} \exp \left(-S_{v_{2}}^{\infty} \tau_{v_{1}} / \eta_{v_{1}}\right)} \geq-\exp (-1)
$$

Solving the equality part of this inequality, we find that

$$
S_{v_{2}}^{\infty}=-\frac{\eta_{v_{2}}}{\tau_{v_{2}}} W\left(-\left(\mathscr{R}_{e, v_{1}}\right)^{\frac{\tau_{v_{2}} \eta_{v_{1}}}{\tau_{v_{1}} \eta_{v_{2}}}} \mathscr{R}_{e, v_{2}} \exp \left(\frac{\tau_{v_{2}} \eta_{v_{1}}}{\tau_{v_{1}} \eta_{v_{2}}}\left(1-\mathscr{R}_{0, v_{1}}\right)\right)\right)
$$

This equation has to be verified both for $W_{0}$ and $W_{-1}$. Let $\Sigma_{0,-1}^{v_{2}}$ be defined by

$$
\Sigma_{0,-1}^{v_{2}}:=-\frac{\eta_{v_{2}}}{\tau_{v_{2}}} W_{0,-1}\left(\mathscr{A}_{v_{2}}\right)
$$

where

$$
\mathscr{A}_{v_{2}}=\left(\mathscr{R}_{e, v_{1}}\right)^{\frac{\tau_{v_{2}} \eta_{v_{1}}}{\tau_{v_{1}} \eta_{v_{2}}}} \mathscr{R}_{e, v_{2}} \exp \left(\frac{\tau_{v_{2}} \eta_{v_{1}}}{\tau_{v_{1}} \eta_{v_{2}}}\left(1-\mathscr{R}_{0, v_{1}}\right)\right)
$$

Then, the domain of $S_{v_{1}}^{\infty}$ as a function of $S_{v_{2}}^{\infty}$ is

$$
S_{v_{2}}^{\infty} \in\left[\min \left(\Sigma_{-1}^{v_{2}}, \Sigma_{0}^{v_{2}}\right), \max \left(\Sigma_{-1}^{v_{2}}, \Sigma_{0}^{v_{2}}\right)\right]
$$

Concerning $S_{v_{2}}^{\infty}$ as a function of $S_{v_{1}}^{\infty}$, we have

$$
S_{v_{1}}^{\infty} \in\left[\min \left(\Sigma_{-1}^{v_{1}}, \Sigma_{0}^{v_{1}}\right), \max \left(\Sigma_{-1}^{v_{1}}, \Sigma_{0}^{v_{1}}\right)\right]
$$

with

$$
\Sigma_{0,-1}^{v_{1}}:=-\frac{\eta_{v_{1}}}{\tau_{v_{1}}} W_{0,-1}\left(\mathscr{A}_{v_{1}}\right)
$$



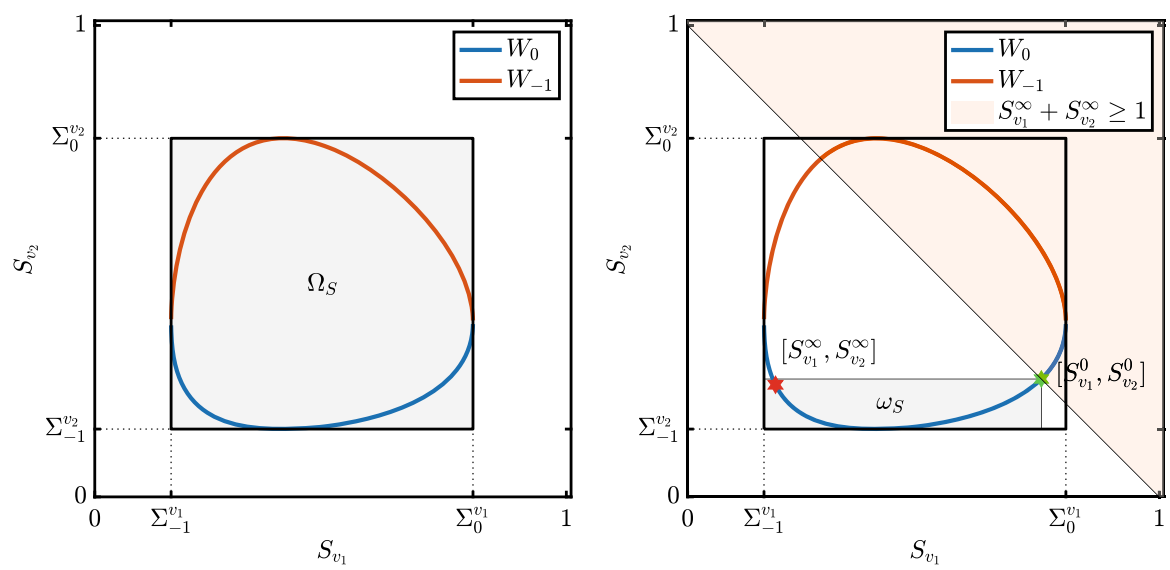

Fig. 4 Location of $S_{v_{1}}^{\infty}$ and $S_{v_{2}}^{\infty}$ together with the visualisation of the domains $\Omega_{S}$ (left) and $\omega_{S}$ (right). The final configuration of susceptible individuals $\left(S_{v_{1}}^{\infty}, S_{v_{2}}^{\infty}\right)$ lies on the closed curve parametrized by the two branches of the Lambert $\mathrm{W}$ function (blue and red curve). We note that $\left(S_{v_{1}}^{\infty}, S_{v_{2}}^{\infty}\right) \in \omega_{S}$ as indicated by the red star on the right figure. Values of the parameters are $d=10^{-3}, \lambda^{1}=\lambda^{2}=6 / 10, \alpha^{1}=\alpha^{2}=1 / 8$, $\tau_{v_{1}}=1, \tau_{v_{2}}=9 / 10, \eta_{v_{1}}=2 / 5, \eta_{v_{2}}=2 / 6$, and initial conditions are set to: $I_{1}^{0}=I_{2}^{0}=10^{-6}$, $S_{0}^{1}=3 / 4-I_{1}^{0}, S_{0}^{2}=1 / 4-I_{1}^{0}$ and $u^{0}(x)=0$. The mass $M^{0}$ is therefore equal to 1 (color figure online)

where

$$
\mathscr{A}_{v_{1}}=\mathscr{R}_{e, v_{1}}\left(\mathscr{R}_{e, v_{2}}\right)^{\frac{\tau_{v_{1}} \eta_{v_{2}}}{\tau_{v_{2}} \eta_{v_{1}}}} \exp \left(\frac{\tau_{v_{1}} \eta_{v_{2}}}{\tau_{v_{2}} \eta_{v_{1}}}\left(1-\mathscr{R}_{0, v_{2}}\right)\right) .
$$

Thus,

$$
\begin{aligned}
\left(S_{v_{1}}^{\infty}, S_{v_{2}}^{\infty}\right) \in \Omega_{S}:= & {\left[\min \left(\Sigma_{-1}^{v_{1}}, \Sigma_{0}^{v_{1}}\right), \max \left(\Sigma_{-1}^{v_{1}}, \Sigma_{0}^{v_{1}}\right)\right] } \\
& \times\left[\min \left(\Sigma_{-1}^{v_{2}}, \Sigma_{0}^{v_{2}}\right), \max \left(\Sigma_{-1}^{v_{2}}, \Sigma_{0}^{v_{2}}\right)\right] .
\end{aligned}
$$

We present on Fig. 4 (left) the functions $W_{0}$ and $W_{-1}$ defining $S_{v_{2}}^{\infty}$ as a function of $S_{v_{1}}^{\infty}$ and the domain $\Omega_{S}$ for a given set of the parameters and initial conditions. We refer to Sect. 5 for details regarding the numerical integration of the model and Sect. 6 for further numerical results on the case of two vertices.

Actually, we can reduce the domain of validity of (4.13)-(4.14) for $S_{v_{1}}^{\infty}$ and $S_{v_{2}}^{\infty}$. Indeed, we know that $S_{v_{k}}, k=1,2$, decay with respect to time, so $S_{v_{k}}^{\infty}<S_{v_{k}}$. Moreover, the sum $S_{v_{1}}^{\infty}+S_{v_{2}}^{\infty}<M^{0}$. Thus, we have

$$
\begin{aligned}
\left(S_{v_{1}}^{\infty}, S_{v_{2}}^{\infty}\right) \in \omega_{S}:= & {\left[\min \left(\Sigma_{-1}^{v_{1}}, \Sigma_{0}^{v_{1}}\right), S_{v_{1}}^{0}\right] } \\
& \times\left[\min \left(\Sigma_{-1}^{v_{2}}, \Sigma_{0}^{v_{2}}\right), S_{v_{2}}^{0}\right] \cap\left\{S_{v_{1}}^{\infty}+S_{v_{2}}^{\infty}<M^{0}\right\} .
\end{aligned}
$$

The domain $\omega_{S}$ is drawn on Fig. 4 (right). 
Concerning $\mathcal{I}_{v_{1}}^{\infty}$ and $\mathcal{I}_{v_{2}}^{\infty}$, we can perform the same analysis. Let

$$
\mathcal{J}_{v_{1}}^{\infty}=-\mathscr{R}_{0, v_{1}}+\frac{\tau_{v_{1}} \eta_{v_{2}}}{\tau_{v_{2}} \eta_{v_{1}}}\left(\mathscr{R}_{e, v_{2}} \exp ^{-\tau_{v_{2}} \mathcal{I}_{v_{2}}^{\infty}}+\tau_{v_{2}} \mathcal{I}_{v_{2}}^{\infty}\right)
$$

and

$$
\mathcal{J}_{v_{2}}^{\infty}=-\mathscr{R}_{0, v_{2}}+\frac{\tau_{v_{2}} \eta_{v_{1}}}{\tau_{v_{1}} \eta_{v_{2}}}\left(\mathscr{R}_{e, v_{1}} \exp ^{-\tau_{v_{1}} \mathcal{I}_{v_{1}}^{\infty}}+\tau_{v_{1}} \mathcal{I}_{v_{1}}^{\infty}\right)
$$

We obtain for $k=1,2$,

$$
\mathcal{I}_{v_{k}}^{\infty}=\frac{1}{\tau_{v_{k}}} W\left(-\mathscr{R}_{e, v_{k}} \exp \left(\mathcal{J}_{v_{k}}^{\infty}\right)\right)-\frac{\mathcal{J}_{v_{k}}^{\infty}}{\tau_{v_{k}}}
$$

still with $W$ equal to $W_{-1}$ and $W_{0}$. Let $\iota_{-1,0}^{1}$ and $\iota_{-1,0}^{2}$ be defined by

$$
\iota_{-1,0}^{v_{1}}=\frac{W_{-1,0}\left(-\mathscr{A}_{v_{1}}\right)}{\tau_{v_{1}}}+\frac{\frac{\tau_{v_{1}} \eta_{v_{2}}}{\tau_{v_{2}} \eta_{v_{1}}}\left(\mathscr{R}_{0, v_{2}}-1-\log \left(\mathscr{R}_{e, v_{2}}\right)\right)}{\tau_{v_{1}}},
$$

and

$$
\iota_{-1,0}^{v_{2}}=\frac{W_{-1,0}\left(-\mathscr{A}_{v_{2}}\right)}{\tau_{v_{2}}}+\frac{\frac{\tau_{v_{2}} \eta_{v_{1}}}{\tau_{v_{1}} \eta_{v_{2}}}\left(\mathscr{R}_{0, v_{1}}-1-\log \left(\mathscr{R}_{e, v_{1}}\right)\right)}{\tau_{v_{2}}},
$$

with $\mathscr{A}_{v_{1}}$ and $\mathscr{A}_{v_{2}}$ given by (4.15) and (4.16). Then,

$$
\left(\mathcal{I}_{v_{1}}^{\infty}, \mathcal{I}_{v_{2}}^{\infty}\right) \in\left[\min \left(\iota_{-1}^{v_{1}}, \iota_{0}^{v_{1}}\right), \max \left(\iota_{-1}^{v_{1}}, \iota_{0}^{v_{1}}\right)\right] \times\left[\min \left(\iota_{-1}^{v_{2}}, \iota_{0}^{v_{2}}\right), \max \left(\iota_{-1}^{v_{2}}, \iota_{0}^{v_{2}}\right)\right] .
$$

We can show that $\min \left(\iota_{-1}^{v_{k}}, \iota_{0}^{v_{k}}\right)<0$ for $k=1,2$. So, we can reduce this domain since $\mathcal{I}_{v_{k}}^{\infty}>0$. So, we define the domain $\omega_{\mathcal{I}}$

$$
\left(\mathcal{I}_{v_{1}}^{\infty}, \mathcal{I}_{v_{2}}^{\infty}\right) \in \omega_{\mathcal{I}}:=\left[0, \max \left(\iota_{-1}^{v_{1}}, \iota_{0}^{v_{1}}\right)\right] \times\left[0, \max \left(\iota_{-1}^{v_{2}}, \iota_{0}^{v_{2}}\right)\right]
$$

As a consequence, we have proved the following lemma.

Lemma 4.5 Case of two vertices. Assume that $c_{\mathcal{V}}=2$ and $c_{\mathcal{E}}=1$, where $c_{\mathcal{E}}$ is the cardinal of $\mathcal{E}$. The final total population of infected individuals at each vertex $\mathcal{I}_{v_{k}}^{\infty}$, $k=1,2$ can be expressed as

$$
\mathcal{I}_{v_{k}}^{\infty}=\frac{1}{\tau_{v_{k}}} W\left(-\mathscr{R}_{e, v_{k}} \exp \left(\mathcal{J}_{v_{k}}^{\infty}\right)\right)-\frac{\mathcal{J}_{v_{k}}^{\infty}}{\tau_{v_{k}}},
$$

with

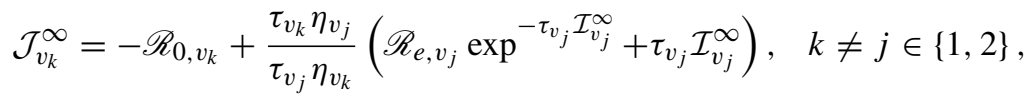



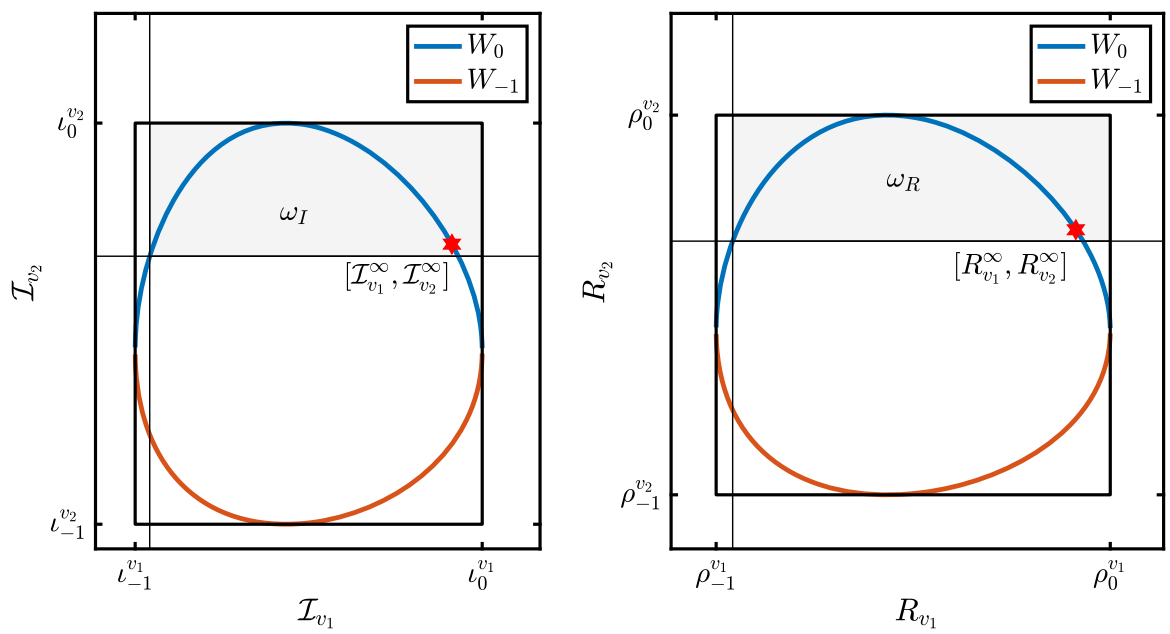

Fig. 5 Location of $\mathcal{I}_{k}^{\infty}$ and $R_{v_{k}}^{\infty}, k=1,2$, and visualisation of the domains $\omega_{\mathcal{I}}$ (left), and domain $\omega_{R}$ (right). In both cases, $\left(\mathcal{I}_{1}^{\infty}, \mathcal{I}_{2}^{\infty}\right) \in \omega_{\mathcal{I}}$ and $\left(R_{1}^{\infty}, R_{2}^{\infty}\right) \in \omega_{R}$ are represented by a red star. Values of the parameters and initial conditions are similar to Fig. 4 (color figure online)

where $\mathscr{R}_{0, v_{k}}:=M^{0} \tau_{v_{k}} / \eta_{v_{k}}$ and $\mathscr{R}_{e, v_{k}}:=S_{v_{k}}^{0} \tau_{v_{k}} / \eta_{v_{k}}, k=1$, 2. Furthermore, we have the sharp bound

$$
\left(\mathcal{I}_{v_{1}}^{\infty}, \mathcal{I}_{v_{2}}^{\infty}\right) \in \omega_{\mathcal{I}}:=\left[0, \max \left(\iota_{-1}^{v_{1}}, \iota_{0}^{v_{1}}\right)\right] \times\left[0, \max \left(\iota_{-1}^{v_{2}}, \iota_{0}^{v_{2}}\right)\right]
$$

with $\iota_{-1,0}^{v_{k}}, k=1,2$ defined in (4.17)-(4.18). See Fig. 5 for an illustration.

Remark 4.6 As the solutions $R_{v_{k}}^{\infty}, k=1,2$, are simply given by $R_{v_{k}}^{\infty}=\eta_{v_{k}} \mathcal{I}_{v_{k}}^{\infty}$, if we let $\rho_{-1,0}^{v_{k}}:=\eta \iota_{-1,0}^{v_{k}}$ then we have

$$
\left(R_{v_{1}}^{\infty}, R_{v_{2}}^{\infty}\right) \in \omega_{R}:=\left[0, \max \left(\rho_{-1}^{v_{1}}, \rho_{0}^{v_{1}}\right)\right] \times\left[0, \max \left(\rho_{-1}^{v_{2}}, \rho_{0}^{v_{2}}\right)\right]
$$

We represent on Fig. 5 the domain $\omega_{R}$.

\section{A semi-implicit numerical scheme which preserves total mass}

In this section, we propose a semi-implicit in time numerical scheme based on finite differences in space which has the property to preserve the discrete total mass.

\subsection{Notations}

For each $e \in \mathcal{E}$, we denote $\delta x_{e}>0$ the space discretization of each edge, and $J_{e} \in \mathbb{N}$ the number of points of the corresponding discretization. For each $i=1, \ldots, J_{e}$, the space grid on each edge is given by $x_{i}=(i-1) \delta x_{e}$ with $\ell_{e}=\left(J_{e}-1\right) \delta x_{e}$. And we 
let $\mathbf{J}:=\sum_{e \in \mathcal{E}} J_{e} \in \mathbb{N}$. Let $\delta t>0$ be the time discretization and denote $t_{m}=m \delta t$ for $m \geq 0$.

For a given function $u \in \mathscr{C}^{1,2}\left(\mathbb{R}_{+} \times \mathcal{G}, \mathbb{R}^{+}\right)$, its space-time discretization is given by some sequence of vectors

$$
u \sim\left(U^{m}\right)_{m \geq 0} \text {, with } U^{m}=\left(U_{1}^{m}, \ldots, U_{\mathbf{J}}^{m}\right)^{\mathbf{t}} \in \mathbb{R}^{\mathbf{J}}
$$

For each $e \in \mathcal{E}$, there exists an integer $j_{e} \in \mathbb{N}$ such that

$$
u_{e}\left(t_{m}, x_{i}\right) \sim U_{j_{e}+i}^{m}, \quad i=1, \ldots, J_{e}, \quad m \geq 0 .
$$

We approximate the laplacian on each edge via finite differences. That is, for each $e \in \mathcal{E}$,

$$
\partial_{x}^{2} u_{e}\left(t_{m}, x_{i}\right) \sim \frac{U_{j_{e}+i-1}^{m}-2 U_{j_{e}+i}^{m}+U_{j_{e}+i+1}^{m}}{\delta x_{e}^{2}}, \quad i=2, \ldots, J_{e}-1, \quad m \geq 1,
$$

where we have only considered the interior points of the discretized domain. Let us now precise how we approximate the laplacian at a given vertex $v \in \mathcal{V}$ of the graph. So let $v \in \mathcal{V}$ such that there are $\delta_{v}$ edges incident to the vertex. We locally label $e \sim v=\left(e_{1}, \ldots, e_{\delta_{v}}\right)$ all these incident edges. For each $v \in \mathcal{V}$, we introduce the map $\sigma_{v}:\left\{e_{1}, \ldots, e_{\delta_{v}}\right\} \rightarrow\{1, \ldots, \mathbf{J}\}$ such that $\sigma_{v}\left(e_{k}\right)$ corresponds to the global index of the grid discretization associated to the vertex $v$ on edge $e_{k}$. Finally, we denote by $n\left(\sigma_{v}\left(e_{k}\right)\right)$ the global index of the nearest neighbor on edge $e_{k}$ to the vertex $v$. Note that either $n\left(\sigma_{v}\left(e_{k}\right)\right)=\sigma_{v}\left(e_{k}\right)-1$ or $n\left(\sigma_{v}\left(e_{k}\right)\right)=\sigma_{v}\left(e_{k}\right)+1$. To approximate the laplacian at a given vertex $v \in \mathcal{V}$ on edge $e_{k}$, we use the following formula

$$
\partial_{x}^{2} u_{e_{k}}\left(t_{m}, v\right) \sim \frac{U_{\sigma_{v}\left(e_{k}\right)}^{*, m}-2 U_{\sigma_{v}\left(e_{k}\right)}^{m}+U_{n\left(\sigma_{v}\left(e_{k}\right)\right)}^{m}}{\delta x_{e_{k}}^{2}}:=\mathcal{Z}_{v, k}^{m}, \quad k=1, \ldots, \delta_{v}, \quad m \geq 1 .
$$

The unknown $U_{\sigma_{v}\left(e_{k}\right)}^{*, m}$ can be expressed by discretization of the boundary condition as follows. For each $v \in \mathcal{V}$ with $e \sim v=\left(e_{1}, \ldots, e_{\delta_{v}}\right)$, we approximate the normal derivative $\partial_{n} u_{e_{k}}\left(t_{m}, v\right)$ as

$$
\partial_{n} u_{e_{k}}\left(t_{m}, v\right) \sim \frac{U_{\sigma_{v}\left(e_{k}\right)}^{*, m}-U_{n\left(\sigma_{v}\left(e_{k}\right)\right)}^{m}}{2 \delta x_{e_{k}}}, \quad k=1, \ldots, \delta_{v}, \quad m \geq 1
$$

Using (2.3), and denoting $I_{v}^{m}$ the time approximation of $I_{v}\left(t_{m}\right)$, we obtain the following expression for $U_{\sigma_{v}\left(e_{k}\right)}^{*, m}$

$$
\begin{aligned}
U_{\sigma_{v}\left(e_{k}\right)}^{*, m}= & U_{n\left(\sigma_{v}\left(e_{k}\right)\right)}^{m}-\frac{2 \delta x_{e_{k}}}{d_{e_{k}}}\left(\alpha_{e_{k}}^{v} U_{\sigma_{v}\left(e_{k}\right)}^{m}+\sum_{l=1}^{\delta_{v}}\left(N_{v}\right)_{k l} U_{\sigma_{v}\left(e_{l}\right)}^{m}\right. \\
& \left.-\lambda_{e_{k}}^{v} I_{v}^{m}\right), \quad k=1, \ldots, \delta_{v}, \quad m \geq 1 .
\end{aligned}
$$


As a consequence, we obtain that for each $k=1, \ldots, \delta_{v}$ and $m \geq 1$

$$
\begin{aligned}
\mathcal{Z}_{v, k}^{m}= & \frac{2 U_{n\left(\sigma_{v}\left(e_{k}\right)\right)}^{m}-2 U_{\sigma_{v}\left(e_{k}\right)}^{m}}{\delta x_{e_{k}}^{2}} \\
& -\frac{2}{d_{e_{k}} \delta x_{e_{k}}}\left(\alpha_{e_{k}}^{v} U_{\sigma_{v}\left(e_{k}\right)}^{m}+\sum_{l=1}^{\delta_{v}}\left(N_{v}\right)_{k l} U_{\sigma_{v}\left(e_{l}\right)}^{m}-\lambda_{e_{k}}^{v} I_{v}^{m}\right) .
\end{aligned}
$$

\subsection{The semi-implicit numerical scheme}

We introduce the following scheme for each $m \geq 0$

$$
\left\{\begin{aligned}
U_{j_{e}+i}^{m+1} & =U_{j_{e}+i}^{m}+d_{e} \delta t\left(\frac{U_{j_{e}+i-1}^{m+1}-2 U_{j_{e}+i}^{m+1}+U_{j_{e}+i+1}^{m+1}}{\delta x_{e}^{2}}\right), \quad i=2, \ldots, J_{e}-1, \quad e \in \mathcal{E}, \\
U_{\sigma_{v}\left(e_{k}\right)}^{m+1} & =U_{\sigma_{v}\left(e_{k}\right)}^{m}+d_{e_{k}} \delta t \mathcal{Z}_{v, k}^{m+1}, \quad k=1, \ldots, \delta_{v}, \quad v \in \mathcal{V} \\
S_{v}^{m+1} & =S_{v}^{m}-\delta t \tau_{v} S_{v}^{m+1} I_{v}^{m} \\
I_{v}^{m+1} & =I_{v}^{m}+\delta t\left(\tau_{v} S_{v}^{m+1} I_{v}^{m}-\eta_{v} I_{v}^{m+1}\right)+\delta t\left(\sum_{k=1}^{\delta_{v}} \alpha_{e_{k}}^{v} U_{\sigma_{v}\left(e_{k}\right)}^{m+1}-\bar{\lambda}_{v} I_{v}^{m+1}\right) \\
R_{v}^{m+1} & =R_{v}^{m}+\delta t \eta_{v} I_{v}^{m+1}
\end{aligned}\right.
$$

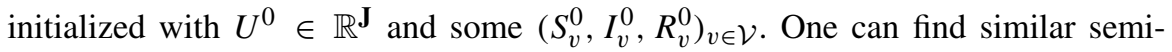
implicit discretization for the SIR part of the model in Sekiguchi and Emiko (2011).

\subsubsection{Well-posedness and positivity}

We prove that the numerical scheme defined through (5.1) is well defined and preserves positivity under some condition on $\delta t$. Indeed, we first remark that the equation for $S_{v}^{m+1}$ and $I_{v}^{m+1}$ in (5.1) can be used to obtain that

$$
\begin{aligned}
S_{v}^{m+1} & =\frac{S_{v}^{m}}{1+\delta t \tau_{v} I_{v}^{m}} \\
I_{v}^{m+1} & =\frac{I_{v}^{m}+\delta t \tau_{v} I_{v}^{m}\left(S_{v}^{m}+I_{v}^{m}\right)}{\left(1+\delta t\left(\eta_{v}+\bar{\lambda}_{v}\right)\right)\left(1+\delta t \tau_{v} I_{v}^{m}\right)}+\frac{\delta t}{1+\delta t\left(\eta_{v}+\bar{\lambda}_{v}\right)} \sum_{l=1}^{\delta_{v}} \alpha_{e_{l}}^{v} U_{\sigma_{v}\left(e_{l}\right)}^{m+1},
\end{aligned}
$$

such that $\mathcal{Z}_{v, k}^{m+1}$ can be expressed only in terms of elements of $U^{m+1}$ as

$$
\begin{aligned}
\mathcal{Z}_{v, k}^{m+1}= & \frac{2 U_{n\left(\sigma_{v}\left(e_{k}\right)\right)}^{m+1}-2 U_{\sigma_{v}\left(e_{k}\right)}^{m+1}}{\delta x_{e_{k}}^{2}} \\
& -\frac{2}{d_{e_{k}} \delta x_{e_{k}}}\left(\alpha_{e_{k}}^{v} U_{\sigma_{v}\left(e_{k}\right)}^{m+1}+\sum_{l=1}^{\delta_{v}}\left(N_{v}\right)_{k l} U_{\sigma_{v}\left(e_{l}\right)}^{m+1}\right.
\end{aligned}
$$




$$
\begin{aligned}
& \left.-\frac{\delta t \lambda_{e_{k}}^{v}}{1+\delta t\left(\eta_{v}+\bar{\lambda}_{v}\right)} \sum_{l=1}^{\delta_{v}} \alpha_{e_{l}}^{v} U_{\sigma_{v}\left(e_{l}\right)}^{m+1}\right) \\
& +\frac{2 \lambda_{e_{k}}^{v}}{d_{e_{k}} \delta x_{e_{k}}}\left(\frac{I_{v}^{m}+\delta t \tau_{v} I_{v}^{m}\left(S_{v}^{m}+I_{v}^{m}\right)}{\left(1+\delta t\left(\eta_{v}+\bar{\lambda}_{v}\right)\right)\left(1+\delta t \tau_{v} I_{v}^{m}\right)}\right) .
\end{aligned}
$$

As a consequence, there exists a matrix $\mathcal{A} \in \mathscr{M}_{\mathbf{J}}(\mathbb{R})$ such that

$$
\left(\mathrm{I}_{\mathbf{J}}+\mathcal{A}\right) U^{m+1}=U^{m}+\mathcal{Y}^{m}
$$

where $\mathcal{Y}^{m} \in \mathbb{R}^{\mathbf{J}}$ is such that

$$
\mathcal{Y}_{j}^{m}=\left\{\begin{array}{cl}
\frac{2 \delta t \lambda_{e_{k}}^{v}}{\delta x_{e_{k}}}\left(\frac{I_{v}^{m}+\delta t \tau_{v} I_{v}^{m}\left(S_{v}^{m}+I_{v}^{m}\right)}{\left(1+\delta t\left(\eta_{v}+\bar{\lambda}_{v}\right)\right)\left(1+\delta t \tau_{v} I_{v}^{m}\right)}\right) & , \text { if } j=\sigma_{v}\left(e_{k}\right), \\
0 & , \text { otherwise. }
\end{array}\right.
$$

Lemma 5.1 There exists a constant $C_{0}>0$, which only depends on the parameters of the system, such that if $0<\delta t<C_{0}$ then we have

- $\mathrm{I}_{\mathbf{J}}+\mathcal{A}$ is invertible;

- if $N_{v}$ is symmetric for each $v \in \mathcal{V}$, then given $V \in \mathbb{R}^{\mathbf{J}}$ with $V \geq \mathbf{0}$, the unique solution $U \in \mathbb{R}^{\mathbf{J}}$ of $\left(\mathrm{I}_{\mathbf{J}}+\mathcal{A}\right) U=V$ also satisfies $U \geq \mathbf{0}$.

Proof Let $U \in \mathbb{R}^{\mathbf{J}} \neq \mathbf{0}$ be such that $\left(\mathrm{I}_{\mathbf{J}}+\mathcal{A}\right) U=0$. Without loss of generality, assume that $U_{j_{0}}=\max _{j=1, \ldots, \mathbf{J}} U_{j}>0$. If there exists $e \in \mathcal{E}$ such that $j_{0}=j_{e}+i_{0}$ for some $i_{0} \in\left\{2, \ldots, J_{e}-1\right\}$, then we have

$$
U_{j_{0}}+\frac{d_{e} \delta t}{\delta x_{e}^{2}}\left(2 U_{j_{0}}-U_{j_{0}-1}-U_{j_{0}+1}\right)=0
$$

which is a contradiction by definition of $U_{j_{0}}$. Next if $j_{0}$ is such that there is $v \in \mathcal{V}$ and $k \in\left\{1, \ldots, \delta_{v}\right\}$ such that $j_{0}=\sigma_{v}\left(e_{k}\right)$, then we have

$$
\begin{aligned}
& U_{\sigma_{v}\left(e_{k}\right)}+\frac{2 d_{e_{k}} \delta t}{\delta x_{e_{k}}^{2}}\left(U_{\sigma_{v}\left(e_{k}\right)}-U_{n\left(\sigma_{v}\left(e_{k}\right)\right)}\right) \\
& =-\frac{2 \delta t}{\delta x_{e_{k}}}\left(\alpha_{e_{k}}^{v} U_{\sigma_{v}\left(e_{k}\right)}+\sum_{l=1}^{\delta_{v}}\left(N_{v}\right)_{k l} U_{\sigma_{v}\left(e_{l}\right)}-\frac{\delta t \lambda_{e_{k}}^{v}}{1+\delta t\left(\eta_{v}+\bar{\lambda}_{v}\right)} \sum_{l=1}^{\delta_{v}} \alpha_{e_{l}}^{v} U_{\sigma_{v}\left(e_{l}\right)}\right) .
\end{aligned}
$$

The left-hand side of the above equality is strictly positive and we claim that the right-hand side is negative. We use the fact that $\left(N_{v}\right)_{k l}=-v_{e_{l}, e_{k}}^{v}$ when $k \neq l$ and $\left(N_{v}\right)_{k k}=\sum_{j \neq k} v_{e_{k}, e_{j}}^{v}$

$$
\sum_{l=1}^{\delta_{v}}\left(N_{v}\right)_{k l} U_{\sigma_{v}\left(e_{l}\right)}=\left(\sum_{j \neq k} v_{e_{k}, e_{j}}^{v}\right) U_{\sigma_{v}\left(e_{k}\right)}-\sum_{l \neq k} v_{e_{l}, e_{k}}^{v} U_{\sigma_{v}\left(e_{l}\right)}
$$




$$
\begin{aligned}
= & {\left[\sum_{j \neq k} v_{e_{k}, e_{j}}^{v}-\sum_{l \neq k} v_{e_{l}, e_{k}}^{v}\right] U_{\sigma_{v}\left(e_{k}\right)} } \\
& +\sum_{l \neq k} v_{e_{l}, e_{k}}^{v}\left(U_{\sigma_{v}\left(e_{k}\right)}-U_{\sigma_{v}\left(e_{l}\right)}\right) .
\end{aligned}
$$

As a consequence, we deduce that

$$
\begin{aligned}
\mathcal{U}_{k}^{v}:= & \alpha_{e_{k}}^{v} U_{\sigma_{v}\left(e_{k}\right)}+\sum_{l=1}^{\delta_{v}}\left(N_{v}\right)_{k l} U_{\sigma_{v}\left(e_{l}\right)}-\frac{\delta t \lambda_{e_{k}}^{v}}{1+\delta t\left(\eta_{v}+\bar{\lambda}_{v}\right)} \sum_{l=1}^{\delta_{v}} \alpha_{e_{l}}^{v} U_{\sigma_{v}\left(e_{l}\right)} \\
= & {\left[\alpha_{e_{k}}^{v}+\sum_{j \neq k} v_{e_{k}, e_{j}}^{v}-\sum_{l \neq k} v_{e_{l}, e_{k}}^{v}-\frac{\delta t \lambda_{e_{k}}^{v}}{1+\delta t\left(\eta_{v}+\bar{\lambda}_{v}\right)} \sum_{l=1}^{\delta_{v}} \alpha_{e_{l}}^{v}\right] U_{\sigma_{v}\left(e_{k}\right)} } \\
& +\sum_{l \neq k} v_{e_{l}, e_{k}}^{v}\left(U_{\sigma_{v}\left(e_{k}\right)}-U_{\sigma_{v}\left(e_{l}\right)}\right) \\
& +\frac{\delta t \lambda_{e_{k}}^{v}}{1+\delta t\left(\eta_{v}+\bar{\lambda}_{v}\right)} \sum_{l=1}^{\delta_{v}} \alpha_{e_{l}}^{v}\left(U_{\sigma_{v}\left(e_{k}\right)}-U_{\sigma_{v}\left(e_{l}\right)}\right)
\end{aligned}
$$

The last two terms are positive by definition of $U_{j_{0}}=U_{\sigma_{v}\left(e_{k}\right)}=\max _{j=1, \ldots, \mathbf{J}} U_{j}>0$. Now using Hypothesis 2.2, we have that

$$
\alpha_{e_{k}}^{v}+\sum_{j \neq k} v_{e_{k}, e_{j}}^{v}-\sum_{l \neq k} v_{e_{l}, e_{k}}^{v}>0
$$

such that the term in bracket is positive provided that

$$
\frac{\delta t \lambda_{e_{k}}^{v}}{1+\delta t\left(\eta_{v}+\bar{\lambda}_{v}\right)} \sum_{l=1}^{\delta_{v}} \alpha_{e_{l}}^{v}<\alpha_{e_{k}}^{v}+\sum_{j \neq k} v_{e_{k}, e_{j}}^{v}-\sum_{l \neq k} v_{e_{l}, e_{k}}^{v}
$$

or equivalently

$$
\begin{aligned}
& \delta t\left[\lambda_{e_{k}}^{v} \sum_{l=1}^{\delta_{v}} \alpha_{e_{l}}^{v}-\left(\eta_{v}+\bar{\lambda}_{v}\right)\left(\alpha_{e_{k}}^{v}+\sum_{j \neq k} v_{e_{k}, e_{j}}^{v}-\sum_{l \neq k} v_{e_{l}, e_{k}}^{v}\right)\right] \\
& <\alpha_{e_{k}}^{v}+\sum_{j \neq k} v_{e_{k}, e_{j}}^{v}-\sum_{l \neq k} v_{e_{l}, e_{k}}^{v} .
\end{aligned}
$$

As a consequence, we impose that

$$
0<\delta t<\min _{v \in \mathcal{V}} \min _{k=1, \ldots, \delta_{v}} \frac{\alpha_{e_{k}}^{v}+\sum_{j \neq k} v_{e_{k}, e_{j}}^{v}-\sum_{l \neq k} v_{e_{l}, e_{k}}^{v}}{\left[\lambda_{e_{k}}^{v} \sum_{l=1}^{\delta_{v}} \alpha_{e_{l}}^{v}-\left(\eta_{v}+\bar{\lambda}_{v}\right)\left(\alpha_{e_{k}}^{v}+\sum_{j \neq k} v_{e_{k}, e_{j}}^{v}-\sum_{l \neq k} v_{e_{l}, e_{k}}^{v}\right)\right]_{+}},
$$


where it is understood that when the positive part is zero there is no condition on $\delta t$. And we have reached a contradiction since

$$
0<U_{\sigma_{v}\left(e_{k}\right)}+\frac{2 d_{e_{k}} \delta t}{\delta x_{e_{k}}^{2}}\left(U_{\sigma_{v}\left(e_{k}\right)}-U_{n\left(\sigma_{v}\left(e_{k}\right)\right)}\right)=-\frac{2 \delta t}{\delta x_{e_{k}}} \mathcal{U}_{k}^{v}<0 .
$$

This shows that $\mathrm{I}_{\mathbf{J}}+\mathcal{A}$ is invertible.

Next let $U \in \mathbb{R}^{\mathbf{J}}$ be the unique solution of $\left(\mathrm{I}_{\mathbf{J}}+\mathcal{A}\right) U=V$ with $V \geq \mathbf{0}$. We denote by $U^{-} \in \mathbb{R}^{\mathbf{J}}$ the vector with components given by

$$
U_{j}^{-}=\min \left(0, U_{j}\right), \quad j=1, \ldots, \mathbf{J} .
$$

Our aim is to evaluate $\left\langle\left(\mathrm{I}_{\mathbf{J}}+\mathcal{A}\right) U, U^{-}\right\rangle_{\mathbf{J}}$ where $\langle\cdot, \cdot\rangle_{\mathbf{J}}$ is the following scalar product on $\mathbb{R}^{\mathbf{J}}$ :

$$
\langle U, V\rangle_{\mathbf{J}}:=\sum_{e \in \mathcal{E}}\left(\sum_{i=2}^{J_{e}-1} U_{j_{e}+i} V_{j_{e}+i}\right)+\frac{1}{2} \sum_{v \in \mathcal{V}}\left(\sum_{k=1}^{\delta_{v}} U_{\sigma_{v}\left(e_{k}\right)} V_{\sigma_{v}\left(e_{k}\right)}\right) .
$$

We divide $\left\langle\left(\mathrm{I}_{\mathbf{J}}+\mathcal{A}\right) U, U^{-}\right\rangle_{\mathbf{J}}$ into three parts:

$$
\left\langle\left(\mathrm{I}_{\mathbf{J}}+\mathcal{A}\right) U, U^{-}\right\rangle_{\mathbf{J}}=\mathcal{Q}_{1}+\mathcal{Q}_{2}+\mathcal{Q}_{3},
$$

where

$$
\begin{aligned}
\mathcal{Q}_{1} & :=\sum_{e \in \mathcal{E}} \sum_{i=2}^{J_{e}-1}\left(U_{j_{e}+i}+\frac{d_{e} \delta t}{\delta x_{e}^{2}}\left(2 U_{j_{e}+i}-U_{j_{e}+i-1}-U_{j_{e}+i+1}\right)\right) U_{j_{e}+i}^{-}, \\
\mathcal{Q}_{2}:= & \frac{1}{2} \sum_{v \in \mathcal{V}} \sum_{k=1}^{\delta_{v}}\left(U_{\sigma_{v}\left(e_{k}\right)}+\frac{2 d_{e_{k}} \delta t}{\delta x_{e_{k}}^{2}}\left(U_{\sigma_{v}\left(e_{k}\right)}-U_{n\left(\sigma_{v}\left(e_{k}\right)\right)}\right)\right) U_{\sigma_{v}\left(e_{k}\right)}^{-} \\
\mathcal{Q}_{3}:= & \delta t \sum_{v \in \mathcal{V}} \sum_{k=1}^{\delta_{v}} \frac{1}{\delta x_{e_{k}}}\left(\alpha_{e_{k}}^{v} U_{\sigma_{v}\left(e_{k}\right)}+\sum_{l=1}^{\delta_{v}}\left(N_{v}\right)_{k l} U_{\sigma_{v}\left(e_{l}\right)}\right. \\
& \left.-\frac{\delta t \lambda_{e_{k}}^{v}}{1+\delta t\left(\eta_{v}+\bar{\lambda}_{v}\right)} \sum_{l=1}^{\delta_{v}} \alpha_{e_{l}}^{v} U_{\sigma_{v}\left(e_{l}\right)}\right) U_{\sigma_{v}\left(e_{k}\right)}^{-} .
\end{aligned}
$$

The first and second terms are handled as follows

$$
\mathcal{Q}_{1}+\mathcal{Q}_{2}=\left\langle U, U^{-}\right\rangle_{\mathbf{J}}+\sum_{e \in \mathcal{E}} \frac{d_{e} \delta t}{\delta x_{e}^{2}} \sum_{i=1}^{J_{e}-1}\left(U_{j_{e}+i+1}-U_{j_{e}+i}\right)\left(U_{j_{e}+i+1}^{-}-U_{j_{e}+i}^{-}\right) \geq 0
$$

For the third term $\mathcal{Q}_{3}$, if we further assume that $N_{v}$ is symmetric, then the matrix $K_{v}=A_{v}+N_{v}$ is symmetric positive definite, and thus for each $v \in \mathcal{V}$ there exists 
some $\beta_{v}>0$ such that

$$
\sum_{k=1}^{\delta_{v}} \frac{1}{\delta x_{e_{k}}}\left(\alpha_{e_{k}}^{v} U_{\sigma_{v}\left(e_{k}\right)}+\sum_{l=1}^{\delta_{v}}\left(N_{v}\right)_{k l} U_{\sigma_{v}\left(e_{l}\right)}\right) U_{\sigma_{v}\left(e_{k}\right)}^{-} \geq \beta_{v} \sum_{k=1}^{\delta_{v}} \frac{1}{\delta x_{e_{k}}} U_{\sigma_{v}\left(e_{k}\right)} U_{\sigma_{v}\left(e_{k}\right)}^{-}
$$

while there exists $\omega_{v}>0$ such that

$$
\left(\sum_{k=1}^{\delta_{v}} \frac{\lambda_{e_{k}}}{\delta x_{e_{k}}} U_{\sigma_{v}\left(e_{k}\right)}^{-}\right)\left(\sum_{l=1}^{\delta_{v}} \alpha_{e_{l}}^{v} U_{\sigma_{v}\left(e_{l}\right)}\right) \leq \omega_{v} \sum_{k=1}^{\delta_{v}} \frac{1}{\delta x_{e_{k}}} U_{\sigma_{v}\left(e_{k}\right)} U_{\sigma_{v}\left(e_{k}\right)}^{-}
$$

And thus, we get an estimate for $\mathcal{Q}_{3}$ of the form

$$
\mathcal{Q}_{3} \geq \delta t \sum_{v \in \mathcal{V}}\left[\left(\beta_{v}-\frac{\delta t \omega_{v}}{1+\delta t\left(\eta_{v}+\bar{\lambda}_{v}\right)}\right) \sum_{k=1}^{\delta_{v}} \frac{1}{\delta x_{e_{k}}} U_{\sigma_{v}\left(e_{k}\right)} U_{\sigma_{v}\left(e_{k}\right)}^{-}\right],
$$

which is positive provided that $\delta t$ is small enough. As a consequence, we have proved that

$$
0 \leq\left\langle\left(\mathrm{I}_{\mathbf{J}}+\mathcal{A}\right) U, U^{-}\right\rangle_{\mathbf{J}}=\left\langle V, U^{-}\right\rangle_{\mathbf{J}} \leq 0
$$

which implies that $U^{-}=\mathbf{0}$ and thus $U \geq \mathbf{0}$.

The previous lemma demonstrates the well-posedness of our numerical scheme (5.1). It also ensures that if we start with positive initial conditions $U^{0} \geq \mathbf{0}$ and $S_{v}^{0}>0, I_{v}^{0} \geq 0$ with $\sum_{v \in \mathcal{V}} I_{v}^{0}>0$ and $R_{v}^{0} \geq 0$, then for all $m \geq 1$ we also have that $U^{m} \geq \mathbf{0}, S_{v}^{m}>0, I_{v}^{m} \geq 0$ and $R_{v}^{m} \geq 0$, provided $\delta t>0$ is small enough and $N_{v}$ is symmetric for each $v \in \mathcal{V}$.

\subsubsection{Preservation of total discrete mass}

For any $U \in \mathbb{R}^{\mathbf{J}}$, we define the following quantity

$$
\operatorname{trap}_{\mathbf{J}}(U):=\sum_{e \in \mathcal{E}} \delta x_{e}\left(\sum_{i=2}^{J_{e}-1} U_{j_{e}+i}\right)+\frac{1}{2} \sum_{v \in \mathcal{V}} \delta x_{e_{k}}\left(\sum_{k=1}^{\delta_{v}} U_{\sigma_{v}\left(e_{k}\right)}\right) .
$$

The expression $\operatorname{trap}_{\mathbf{J}}(U)$ is simply the trapezoidal rule applied to the elements of $U$ adapted to our graph $\mathcal{G}$. From (5.1), we get that

$$
\begin{aligned}
\operatorname{trap}_{\mathbf{J}}\left(U^{m+1}\right)= & \operatorname{trap}_{\mathbf{J}}\left(U^{m}\right)+\delta t \sum_{e \in \mathcal{E}} \frac{d_{e}}{\delta x_{e}}\left(\sum_{i=2}^{J_{e}-1}\left(U_{j_{e}+i-1}^{m+1}-2 U_{j_{e}+i}^{m+1}+U_{j_{e}+i+1}^{m+1}\right)\right) \\
& +\frac{\delta t}{2} \sum_{v \in \mathcal{V}} \delta x_{e_{k}} d_{e_{k}}\left(\sum_{k=1}^{\delta_{v}} \mathcal{Z}_{v, k}^{m+1}\right) .
\end{aligned}
$$


Upon denoting $\mathcal{Z}_{v, k}^{1, m}$ the following quantity

$$
\mathcal{Z}_{v, k}^{1, m}:=\frac{2 U_{n\left(\sigma_{v}\left(e_{k}\right)\right)}^{m}-2 U_{\sigma_{v}\left(e_{k}\right)}^{m}}{\delta x_{e_{k}}^{2}}
$$

we get that

$$
\frac{1}{2} \sum_{v \in \mathcal{V}} \delta x_{e_{k}} d_{e_{k}}\left(\sum_{k=1}^{\delta_{v}} \mathcal{Z}_{v, k}^{1, m+1}\right)=\sum_{v \in \mathcal{V}} \frac{d_{e_{k}}}{\delta x_{e_{k}}}\left(\sum_{k=1}^{\delta_{v}} U_{n\left(\sigma_{v}\left(e_{k}\right)\right)}^{m+1}-U_{\sigma_{v}\left(e_{k}\right)}^{m+1}\right)
$$

Next, we observe that

$$
\begin{gathered}
\sum_{e \in \mathcal{E}} \frac{d_{e}}{\delta x_{e}}\left(\sum_{i=2}^{J_{e}-1}\left(U_{j_{e}+i-1}^{m+1}-2 U_{j_{e}+i}^{m+1}+U_{j_{e}+i+1}^{m+1}\right)\right) \\
+\sum_{v \in \mathcal{V}} \frac{d_{e_{k}}}{\delta x_{e_{k}}}\left(\sum_{k=1}^{\delta_{v}} U_{n\left(\sigma_{v}\left(e_{k}\right)\right)}^{m+1}-U_{\sigma_{v}\left(e_{k}\right)}^{m+1}\right)=0,
\end{gathered}
$$

where the cancellation comes from the specific structure of the discretized laplacian through finite differences. As a consequence, we have that

$$
\begin{aligned}
\operatorname{trap}_{\mathbf{J}}\left(U^{m+1}\right)= & \operatorname{trap}_{\mathbf{J}}\left(U^{m}\right) \\
& -\delta t \sum_{v \in \mathcal{V}} \sum_{k=1}^{\delta_{v}}\left(\alpha_{e_{k}}^{v} U_{\sigma_{v}\left(e_{k}\right)}^{m+1}+\sum_{l=1}^{\delta_{v}}\left(N_{v}\right)_{k l} U_{\sigma_{v}\left(e_{l}\right)}^{m+1}-\lambda_{e_{k}}^{v} I_{v}^{m+1}\right) .
\end{aligned}
$$

We also have that

$$
\sum_{k=1}^{\delta_{v}} \sum_{l=1}^{\delta_{v}}\left(N_{v}\right)_{k l} U_{\sigma_{v}\left(e_{l}\right)}^{m+1}=\sum_{l=1}^{\delta_{v}}\left(\sum_{k=1}^{\delta_{v}}\left(N_{v}\right)_{k l}\right) U_{\sigma_{v}\left(e_{l}\right)}^{m+1}=0
$$

as the sum over the lines of $N_{v}$ vanishes. And thus we get

$$
\operatorname{trap}_{\mathbf{J}}\left(U^{m+1}\right)=\operatorname{trap}_{\mathbf{J}}\left(U^{m}\right)-\delta t \sum_{v \in \mathcal{V}}\left(\sum_{k=1}^{\delta_{v}} \alpha_{e_{k}}^{v} U_{\sigma_{v}\left(e_{k}\right)}^{m+1}-\bar{\lambda}_{v} I_{v}^{m+1}\right) .
$$


On the other hand, from (5.1) we also have

$$
\begin{aligned}
\sum_{v \in \mathcal{V}}\left(S_{v}^{m+1}+I_{v}^{m+1}+R_{v}^{m+1}\right)= & \sum_{v \in \mathcal{V}}\left(S_{v}^{m}+I_{v}^{m}+R_{v}^{m}\right) \\
& +\delta t \sum_{v \in \mathcal{V}}\left(\sum_{k=1}^{\delta_{v}} \alpha_{e_{k}}^{v} U_{\sigma_{v}\left(e_{k}\right)}^{m+1}-\bar{\lambda}_{v} I_{v}^{m+1}\right)
\end{aligned}
$$

As a conclusion, we have proved the following result.

Lemma 5.2 Let $\left(U^{m}, S_{v}^{m}, I_{v}^{m}, R_{v}^{m}\right)$ a solution of (5.1), then we have for each $m \geq 0$ $\operatorname{trap}_{\mathbf{J}}\left(U^{m+1}\right)+\sum_{v \in \mathcal{V}}\left(S_{v}^{m+1}+I_{v}^{m+1}+R_{v}^{m+1}\right)=\operatorname{trap}_{\mathbf{J}}\left(U^{m}\right)+\sum_{v \in \mathcal{V}}\left(S_{v}^{m}+I_{v}^{m}+R_{v}^{m}\right)$

This is the discrete conter part of conservation of mass for the continuous model. Now, combining Lemmas 5.1-5.2, we have proved the following theorem.

Theorem 3 There exists a constant $C_{0}>0$, which only depends on the parameters of the system, such that if $0<\delta t<C_{0}$, then the numerical scheme (5.1) defines a unique sequence $\left(U^{m}, S_{v}^{m}, I_{v}^{m}, R_{v}^{m}\right)_{m \geq 0}$. If we further assume that $N_{v}$ is symmetric for each $v \in \mathcal{V}$, then the numerical scheme (5.1) preserves the positivity of the initial condition. Finally, for each solution of (5.1), the total discrete mass is preserved, namely for each $m \geq 0$, we have

$$
\begin{gathered}
\operatorname{trap}_{\mathbf{J}}\left(U^{m+1}\right)+\sum_{v \in \mathcal{V}}\left(S_{v}^{m+1}+I_{v}^{m+1}+R_{v}^{m+1}\right) \\
=\operatorname{trap}_{\mathbf{J}}\left(U^{m}\right)+\sum_{v \in \mathcal{V}}\left(S_{v}^{m}+I_{v}^{m}+R_{v}^{m}\right) .
\end{gathered}
$$

\section{Numerical results for a selection of graphs}

In the present section, we illustrate our theoretical results with a collection of numerical simulations for various types of graphs. Throughout this section the time discretization is set to $\delta t=0.01$ while the space discretization to $\delta_{x_{e}}=0.01$ for each $e \in \mathcal{E}$.

\subsection{Case of 2 vertices and 1 edge}

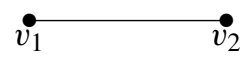

We first consider the case where $c_{\mathcal{V}}=2$ and $c_{\mathcal{E}}=1$, where $c_{\mathcal{E}}$ denotes the cardinal of $\mathcal{E}$. In this setting, we recall that our model reads as follows

$$
\partial_{t} u(t, x)=d \partial_{x}^{2} u(t, x), \quad t>0, \quad x \in(0, \ell),
$$


with boundary conditions

$$
\left\{\begin{array}{rl}
-d \partial_{x} u(t, 0)+\alpha_{1} u(t, 0) & =\lambda_{1} I_{1}(t), \\
d \partial_{x} u(t, \ell)+\alpha_{2} u(t, \ell) & =\lambda_{2} I_{2}(t)
\end{array} \quad t>0,\right.
$$

where $\left(S_{i}(t), I_{i}(t), R_{i}(t)\right)$, for $i=1,2$, solution of

$$
\left\{\begin{aligned}
S_{i}^{\prime}(t) & =-\tau_{i} S_{i}(t) I_{i}(t) \\
I_{i}^{\prime}(t) & =\tau_{i} S_{i}(t) I_{i}(t)-\eta_{i} I_{v}(t)+\alpha_{i} u\left(t, v_{i}\right)-\lambda_{i} I_{i}(t), \quad t>0 \\
R_{i}^{\prime}(t) & =\eta_{i} I_{i}(t)
\end{aligned}\right.
$$

where $v_{1}=0$ and $v_{2}=\ell$. This system is complemented by some initial condition $\left(u^{0}, S_{i}^{0}, I_{i}^{0}, R_{i}^{0}\right)$ with $S_{i}>0, I_{1}^{0}+I_{2}^{0}>0, R_{i}^{0}=0$ and $u^{0} \geq 0$ such that the boundary condition is satisfied initially. Finally, we normalize the total mass as follows

$$
M^{0}=\int_{0}^{\ell} u^{0}(x) \mathrm{d} x+\sum_{i=1}^{2}\left(S_{i}^{0}+I_{i}^{0}\right)=1 .
$$

For the numerical simulations, we have fixed initial conditions to be of the form

$$
u^{0}(x)=\frac{\lambda_{1} I_{0}}{\alpha_{1}} \exp \left(-\frac{\alpha_{2} x^{2}}{2 d \ell}\right), x \in[0, \ell]
$$

with

$$
\left(S_{1}^{0}, I_{1}^{0}, S_{2}^{0}, I_{2}^{0}\right)=\left(S_{0}-I_{0}-\int_{0}^{\ell} u^{0}(x) \mathrm{d} x, I_{0}, 1-S_{0}, 0\right)
$$

where $S_{0}$ and $I_{0}$ may vary. In Figs. 6,7 and $8, S_{0}$ and $I_{0}$ are fixed to $\left(S_{0}, I_{0}\right)=$ $\left(1 / 2,10^{-6}\right)$, while in Fig. $10, S_{0}$ is allowed to vary and $I_{0}$ is fixed to $I_{0}=10^{-6}$.

In Fig. 6, we report the profiles of the solutions $\left(S_{i}(t), I_{i}(t)\right)$ together with the total population on the edge $\int_{0}^{\ell} u(t, x) \mathrm{d} x$ and the total mass of the system $M(t)$ as the parameter $\lambda_{1}$ is varied from 0.05 to 0.95 , while all other parameters are being kept fixed. We observe that the dynamics of the epidemic at the second vertex is almost independent of the parameter $\lambda_{1}$ while it has a significant impact on the dynamics at the first vertex. Indeed, as $\lambda_{1}$ is increased, the maximum of infected individuals $\max _{t \geq 0} I_{1}(t)$ is decreased. In the last panel of the figure, we also illustrate the conservation of total population where the fluctuations around $M^{0}=1$ is of order $10^{-12}$. In the top panel of Fig. 7, we present the final total populations of infected individuals and corresponding final population of susceptible individuals as $\lambda_{1}$ is varied. The blue curve is the location of $\left(\mathcal{I}_{1}^{\infty}, \mathcal{I}_{2}^{\infty}\right)$ respectively $\left(S_{1}^{\infty}, S_{2}^{\infty}\right)$ while the dark red circles indicate the numerically computed values. We recover the fact that $\lambda_{1}$ has a more significant impact on the final total populations at the first vertex than it has at the second vertex. To get a better understanding of the intricate dynamics between the 

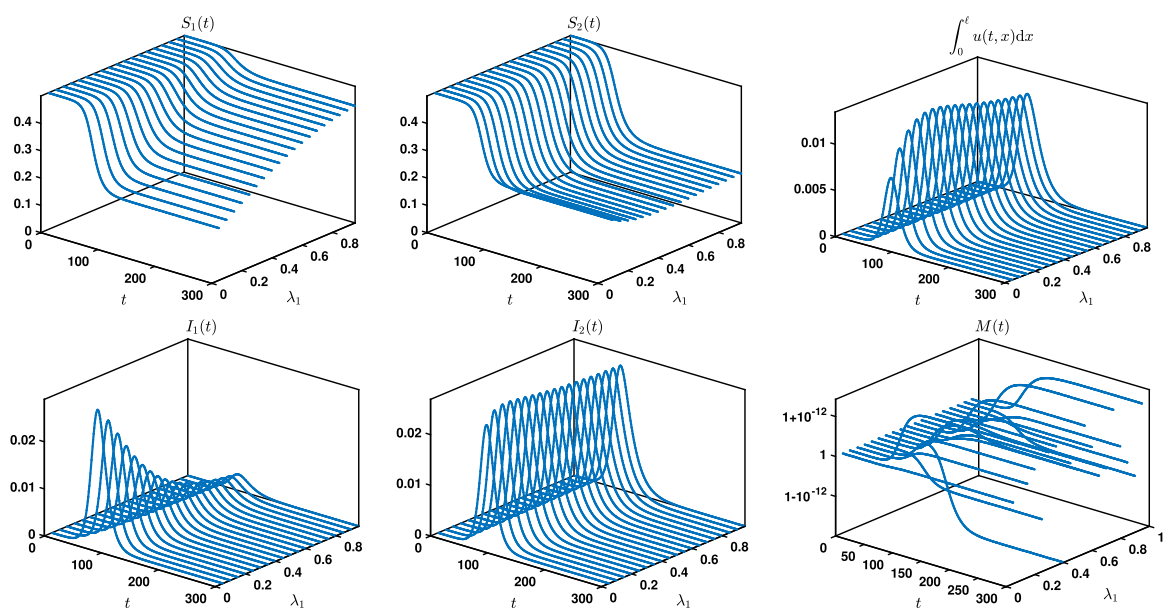

Fig. 6 Profiles of the solutions $\left(S_{i}(t), I_{i}(t)\right)$ together with the total population on the edge $\int_{0}^{\ell} u(t, x) \mathrm{d} x$ and the total mass of the system $M(t)$ as the parameter $\lambda_{1}$ is varied from 0.05 to 0.95 . All other parameters are fixed and set to $d=\ell=1, \lambda_{2}=1 / 10, \alpha_{1}=\alpha_{2}=1 / 4$, and $\tau_{1}=\tau_{2}=1$ with $\eta_{1}=\eta_{2}=1 / 3$. For the initial condition we have $\left(S_{0}, I_{0}\right)=\left(1 / 2,10^{-6}\right)$

epidemic at the two vertices, we also present the relative distance $\Delta \mathcal{T}:=\mathcal{T}_{2}-\mathcal{T}_{1}$ between time of maximal infection $\mathcal{T}_{j}$ in each population as $\lambda_{1}$ is varied. We observe that $\Delta \mathcal{T}$ is not monotone in $\lambda_{1}$, as it first decreases and then increases. But we also note that $\Delta \mathcal{T}<0$ for $\lambda_{1} \geq 0.1$ traducing the fact that the pick of the epidemic occurs at the second vertex before it does at the first vertex, although initially $I_{2}^{0}=0$. This illustrates the effect of the diffusion of infected individuals along the edge.

Similarly, in Fig. 7, we report the final total populations of infected individuals and corresponding final population of susceptible individuals as $\lambda_{2}$ (second panel), $\alpha_{1}$ (third panel) and $\alpha_{2}$ (bottom panel) are varied from 0.05 to 0.95 . As expected, the final total population of infected individuals at the second vertex decreases as $\lambda_{2}$ increases while at the first vertex it varies less significantly. As $\alpha_{1}$ increases, the final total population of infected individuals at the first vertex increases while it decreases at the second vertex. This time the relative distance $\Delta \mathcal{T}:=\mathcal{T}_{2}-\mathcal{T}_{1}$ between time of maximal infection is monotonically increasing with $\alpha_{1}$. We get the opposite monotonicity properties as $\alpha_{2}$ is varied.

In Fig. 8, we investigate the joint effect of the diffusion coefficient $d$ and the length of the edge $\ell$ on the dynamics of the epidemic at the vertices. Here, we focus on the delay between time of maximal infection $\mathcal{T}_{j}$ in each infected population $I_{j}(t)$. As expected, when the diffusion coefficient is really small while the length is being kept at order one, $\Delta \mathcal{T}$ takes large value: $\Delta \mathcal{T} \sim 10^{4}$ when $d=10^{-3}$ and $\ell=1$. Biologically, this means that when the diffusion coefficient is really small it takes more time for infected individuals from vertex one to reach the second vertex and start an epidemic. We also note that at fixed $\ell, \Delta \mathcal{T}$ monotonically decreases as $d$ increases, while at fixed $d, \Delta \mathcal{T}$ monotonically increases as $\ell$ increases.

In Figs. 9 and 10, we vary respectively the initial population of susceptible individuals $S_{0}$ and infected individuals $I_{0}$. We visualize the final total populations of infected 

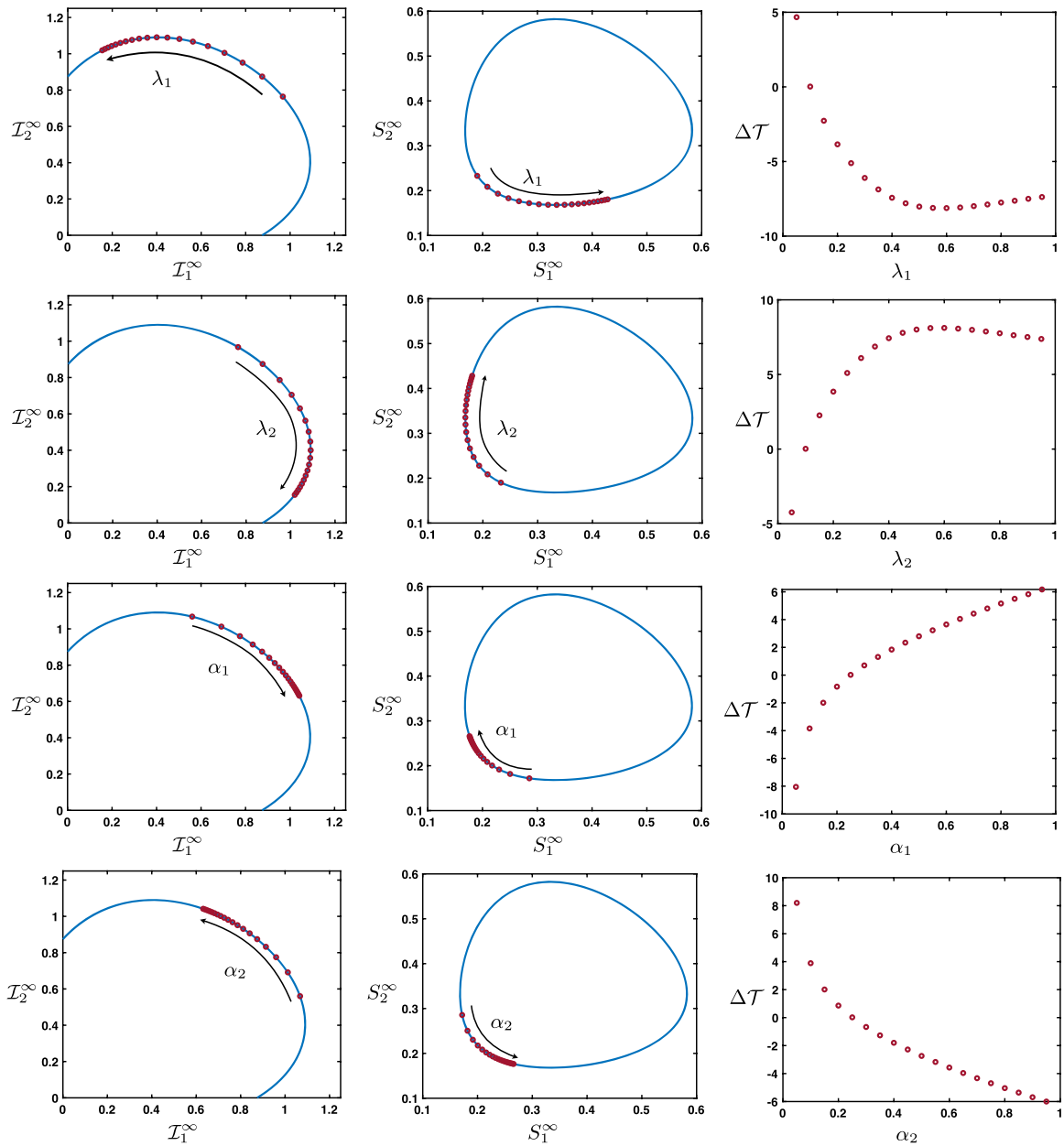

Fig. 7 Final total populations of infected individuals (left) and corresponding final population of susceptible individuals (middle) as one parameter is varied from 0.05 to 0.95 while all parameters are fixed. The blue curve is the location of $\left(\mathcal{I}_{1}^{\infty}, \mathcal{I}_{2}^{\infty}\right)$ respectively $\left(S_{1}^{\infty}, S_{2}^{\infty}\right)$ while the dark red circles indicate the numerically computed values. Right: relative distance $\Delta \mathcal{T}:=\mathcal{T}_{2}-\mathcal{T}_{1}$ between time of maximal infection $\mathcal{T}_{j}$ in each population, indicated by dark red circles, as the parameter is varied from 0.05 to 0.95 . Varying parameters: $\lambda_{1}$ (top panel), $\lambda_{2}$ (second panel), $\alpha_{1}$ (third panel) and $\alpha_{2}$ (bottom panel) (color figure online)

individuals and corresponding final population of susceptible individuals on the parameterized surfaces $\left(\mathcal{I}_{1}^{\infty}, \mathcal{I}_{2}^{\infty}, S_{0}\right)$ and $\left(S_{1}^{\infty}, S_{2}^{\infty}, S_{0}\right)$, respectively $\left(\mathcal{I}_{1}^{\infty}, \mathcal{I}_{2}^{\infty}, I_{0}\right)$ and $\left(S_{1}^{\infty}, S_{2}^{\infty}, I_{0}\right)$, where the level sets of the parameterized surface are given by the conservation of total mass (4.7). We note that $\left(\mathcal{I}_{1}^{\infty}, \mathcal{I}_{2}^{\infty}\right)$ and $\left(S_{1}^{\infty}, S_{2}^{\infty}\right)$ are almost independent of $I_{0}$ when $I_{0} \leq 10^{-3}$ with sensible variations only occurring for larger values of $I_{0}$. On the other hand, we observe that as $S_{0}$ is increased the final total population of infected individuals increases at the first vertex while it decreases at the second one. The dependence of $\left(S_{1}^{\infty}, S_{2}^{\infty}\right)$ as a function of $S_{0}$ is more subtile and is presented in Fig. 11. In the same figure, we also show the location of $\max I_{j}(t)$ 


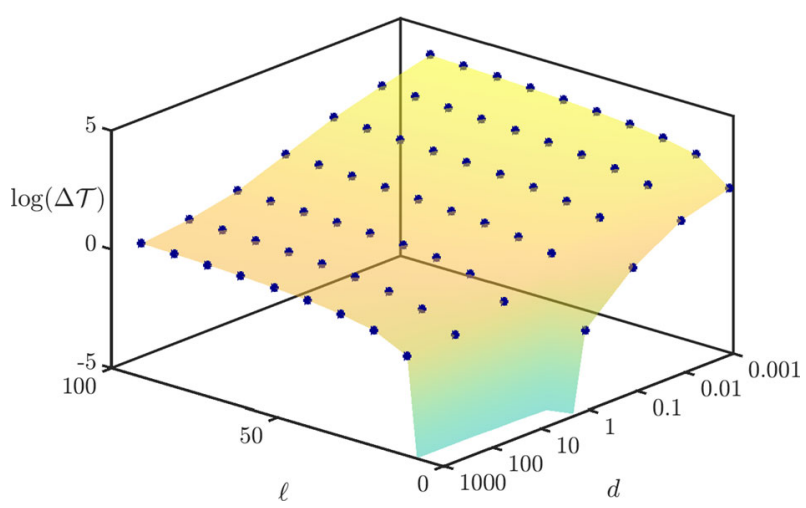

Fig. 8 Log-plot of the relative distance $\Delta \mathcal{T}=\mathcal{T}_{2}-\mathcal{T}_{1}$ between time of maximal infection $\mathcal{T}_{j}$ in each population $I_{j}(t)$ as the diffusion coefficient $d$ and the length of the edge $\ell$ are varied while all other parameters are fixed to $\lambda_{1}=\lambda_{2}=1 / 10, \alpha_{1}=\alpha_{2}=1 / 4$, and $\tau_{1}=\tau_{2}=1$ with $\eta_{1}=\eta_{2}=1 / 3$. For the initial condition we have $\left(S_{0}, I_{0}\right)=\left(1 / 2,10^{-6}\right)$. We note that as $d$ becomes smaller $\Delta \mathcal{T}$ rapidly increases as $\ell$ increases
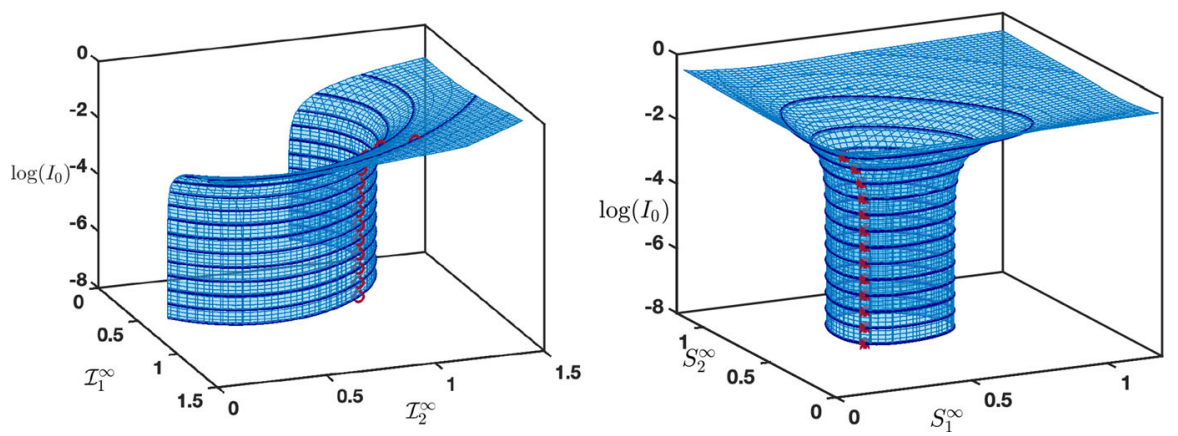

Fig. 9 Final total populations of infected individuals (left) and corresponding final population of susceptible individuals (right) as the initial population of susceptible individuals $I_{0}$ is varied from $10^{-7}$ to $10^{-1}$ in logscale while $S_{0}=1 / 2$ is fixed. The dark blue curves are the location of $\left(\mathcal{I}_{1}^{\infty}, \mathcal{I}_{2}^{\infty}\right)$ respectively $\left(S_{1}^{\infty}, S_{2}^{\infty}\right)$ for each value of $S_{0}$, while the dark red circles indicate the numerically computed values. Each dark blue curve is a level set of the parameterized surface given by the conservation of total mass (4.7). All other parameters are fixed to $d=\ell=1, \lambda_{1}=\lambda_{2}=1 / 10, \alpha_{1}=\alpha_{2}=1 / 4$, and $\tau_{1}=\tau_{2}=1$ with $\eta_{1}=\eta_{2}=1 / 3$ (color figure online)

and its amplitude. We observe a strong nonlinear dependence with respect to $S_{0}$. As $S_{0}$ increases, we first see that the time at which $I_{1}(t)$ is maximal increases and then decreases, while $\max I_{1}(t)$ is monotonically increasing. The converse is observed at the second vertex. 

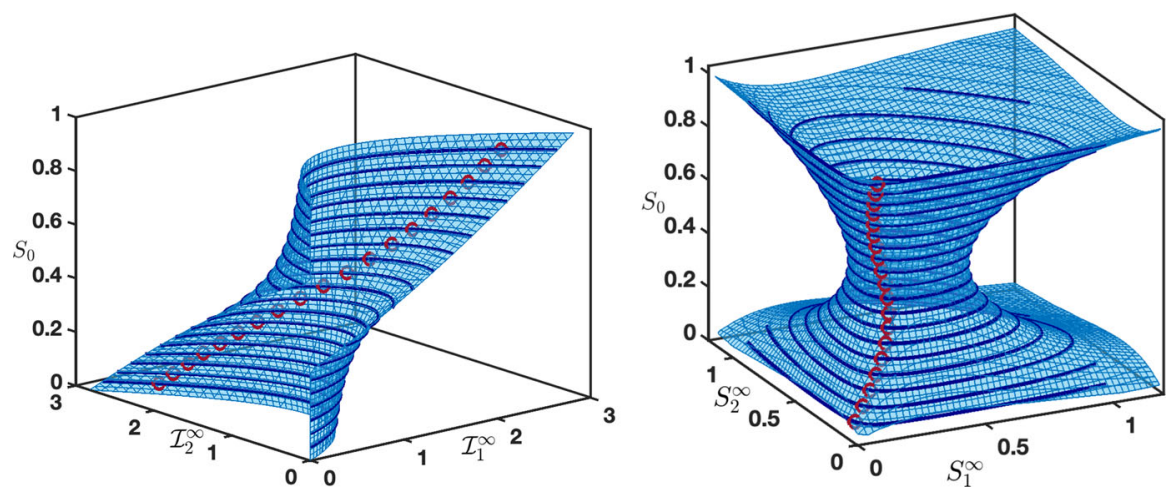

Fig. 10 Final total populations of infected individuals (left) and corresponding final population of susceptible individuals (right) as the initial population of susceptible individuals $S_{0}$ is varied from 0.05 to 0.95 while $I_{0}=10^{-6}$ is fixed. The dark blue curves are the location of $\left(\mathcal{I}_{1}^{\infty}, \mathcal{I}_{2}^{\infty}\right)$ respectively $\left(S_{1}^{\infty}, S_{2}^{\infty}\right)$ for each value of $S_{0}$, while the dark red circles indicate the numerically computed values. Each dark blue curve is a level set of the parameterized surface given by the conservation of total mass (4.7). All other parameters are fixed to $d=\ell=1, \lambda_{1}=\lambda_{2}=1 / 10, \alpha_{1}=\alpha_{2}=1 / 4$, and $\tau_{1}=\tau_{2}=1$ with $\eta_{1}=\eta_{2}=1 / 3$ (color figure online)
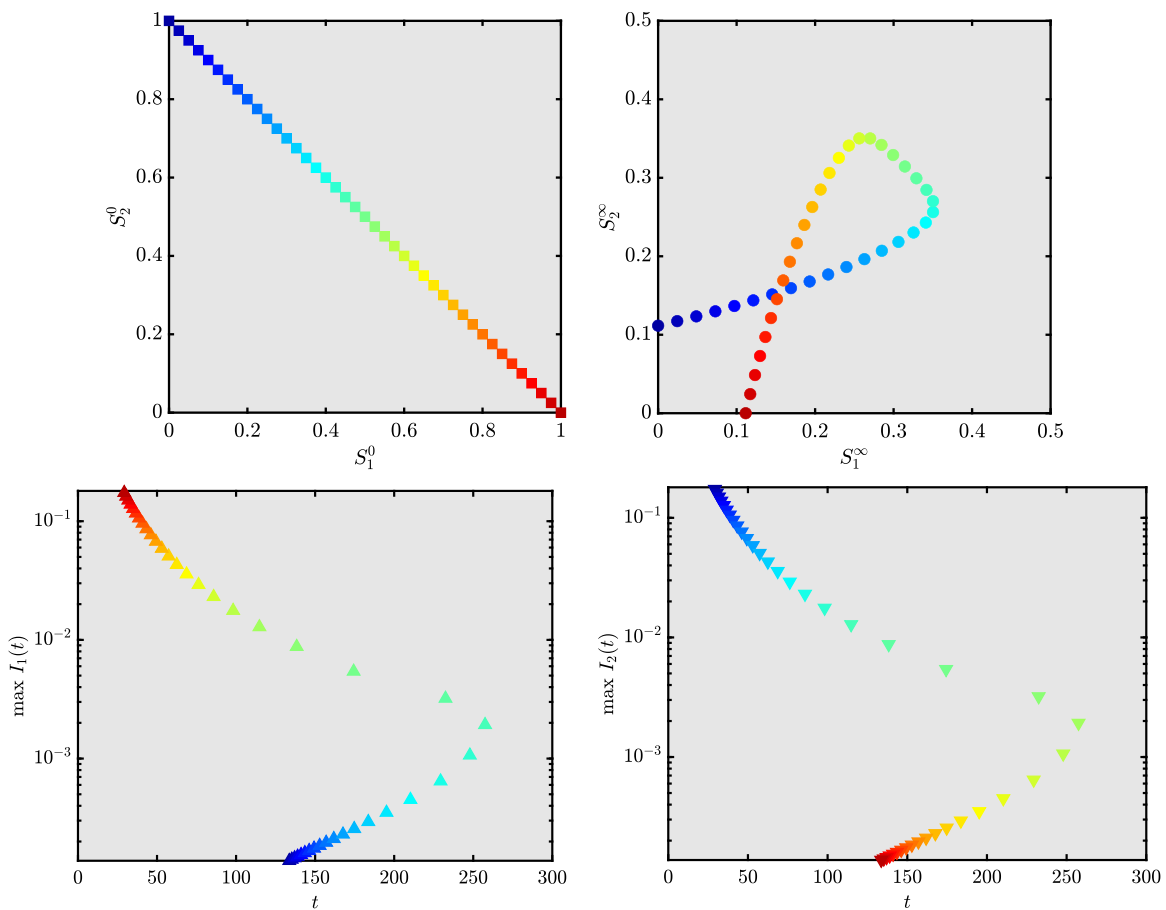

Fig. 11 Locations of $S_{1,2}^{\infty}$ (top) and $\max I_{1,2}(t)$ (bottom left and right) as functions of $S_{1,2}^{0}$. Values of all other parameters are similar to Fig. 10 . The initial condition is of the form $\left(S_{0}, 1-S_{0}\right)$ with $S_{0} \in[0,1]$ and to each initial configuration is associated a color code from blue to red. The curve in the top right panel is a projection on the $\left(S_{1}^{\infty}, S_{2}^{\infty}\right)$-plane of the parametrized a curve from Fig. 10, right panel (color figure online) 


\subsection{Case of 3 vertices and 3 edges}

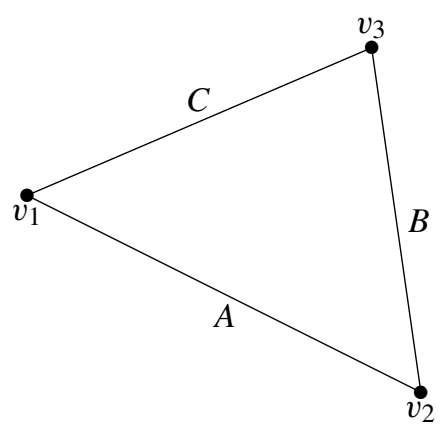

Next, we consider the case of 3 vertices and 3 edges arranged in a triangular configuration. For the numerical simulations presented in Fig. 12, we have assumed full symmetry in the parameters that is

$$
\begin{aligned}
& \left(\ell_{e}, d_{e}\right)=(\ell, d), \quad e \in \mathcal{E}, \quad\left(\tau_{v}, \eta_{v}\right)=(\tau, \eta), \quad v \in \mathcal{V}, \\
& \left(\alpha_{e}^{v}, \lambda_{e}^{v}\right)=(\alpha, \lambda), \quad(e, v) \in \mathcal{E} \times \mathcal{V}, \quad v_{e, e^{\prime}}^{v}=v, \quad\left(e, e^{\prime}, v\right) \in \mathcal{E} \times \mathcal{E} \times \mathcal{V} .
\end{aligned}
$$

Regarding the initial condition, we have chosen

$$
\left(S_{1}^{0}, I_{1}^{0}, S_{2}^{0}, I_{2}^{0}, S_{3}^{0}, I_{3}^{0}\right)=\left(S^{0}-I_{0}, I_{0}, S^{0}, 0, S^{0}, 0\right) \quad v \in \mathcal{V},
$$

for a given $\left(S^{0}, I_{0}\right)$, while for each $e \in \mathcal{E}$ we have set $u_{e}^{0}(x)=0$ on $\Omega_{e}$. Note that, we have initially a boundary layer as our initial condition does not satisfy (2.3) for small times. We remark that the final total populations of infected individuals and corresponding final population of susceptible individuals belong to a surface as provided by (4.7)-(4.8) from Theorem 2.

In Fig. 13, we tested a different configuration. Upon labeling by $A$ the edge between vertices $v_{1}$ and $v_{2}, B$ the edge between vertices $v_{2}$ and $v_{3}$ and $C$ the edge between vertices $v_{1}$ and $v_{3}$, we have set the parameters to

$$
\alpha_{A}^{1}=\alpha_{B}^{2}=\alpha_{C}^{3}=0 \text {, and } \alpha_{A}^{2}=\alpha_{B}^{3}=\alpha_{C}^{2}=1 / 10,
$$

while

$$
\lambda_{A}^{1}=\lambda_{B}^{2}=\lambda_{C}^{3}=1 / 20, \text { and } \lambda_{A}^{2}=\lambda_{B}^{3}=\lambda_{C}^{2}=0,
$$

and

$$
v_{A, C}^{1}=v_{B, A}^{2}=v_{C, B}^{3}=0, \text { and } v_{C, A}^{1}=v_{A, B}^{2}=v_{B, C}^{3}=1 / 30 .
$$

The length of each edge is fixed $\ell_{e}=\ell=1$ and $\left(\tau_{v}, \eta_{v}\right)=(1,1 / 7)$ at each vertex $v \in \mathcal{V}$. Finally, we have set different coefficients on each edge, namely $d_{A}=1$, 

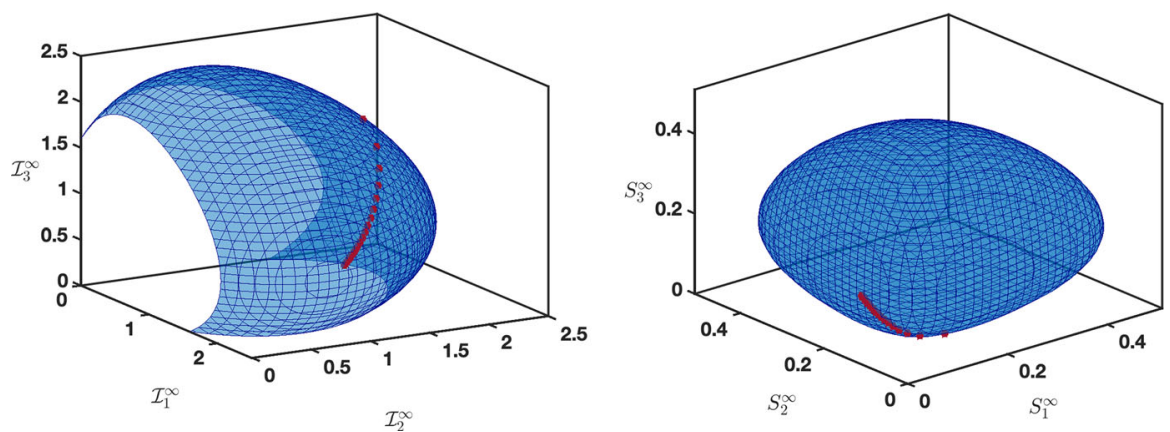

Fig. 12 Final total populations of infected individuals (left) and corresponding final population of susceptible individuals (right) as $v$ is varied from 0.05 to 0.95 . The dark blue surfaces are the location of $\left(\mathcal{I}_{1}^{\infty}, \mathcal{I}_{2}^{\infty}, \mathcal{I}_{3}^{\infty}\right)$ respectively $\left(S_{1}^{\infty}, S_{2}^{\infty}, S_{3}^{\infty}\right)$ for each value of $S_{0}$, while the dark red circles indicate the numerically computed values. Parameters were set to $\ell=d=1,(\tau, \eta)=(1,1 / 6)$, and $(\alpha, \lambda)=(1 / 8,1 / 10)$, while the initial condition is $\left(S_{0}, I_{0}\right)=\left(1,10^{-6}\right)$ (color figure online)
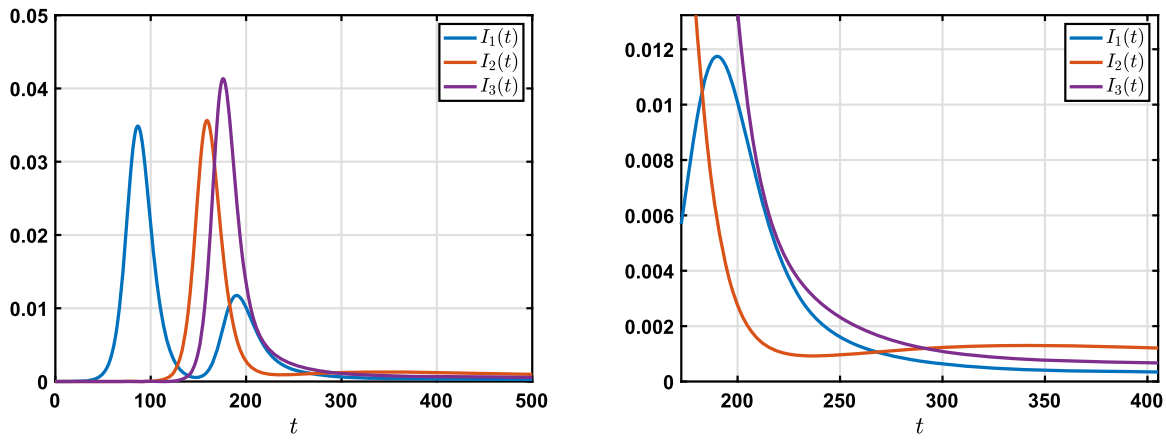

Fig. 13 Time plot of infected populations in the case of 3 vertices and 3 edges in a triangular configuration between times [0, 500] (left) and a zoom for times between [150, 400] (right). We observe a second wave of infection at the first vertex resulting from incoming infected individuals that have successively passed through the two other vertices. This second wave is also present at the second vertex with a slight increase of $I_{2}(t)$ after the second wave has reached the first vertex. Parameters values are set in the text

$d_{B}=10^{-2}$ and $d_{C}=10^{-3}$. Initially, we assume that infected individuals are only present at vertex $v_{1}$ and each vertex has the same number of susceptible individuals fixed to $1 / 3$. Finally, for each $e \in \mathcal{E}$ we have set $u_{e}^{0}(x)=0$ on $\Omega_{e}$. We see in Fig. 13 that such a configuration can generate a second wave of infection at the first and second vertices showing that transient dynamics can be complex with multiple bumps of infection. 


\subsection{Case of 4 vertices and 3 edges}

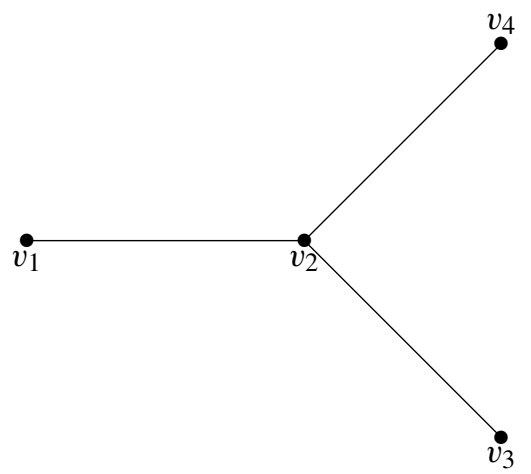

Next, we consider a star-shape graph with 4 vertices and 3 edges where one vertex is connected to the three others. In this configuration, we assume that our parameters may vary with respect to time, modeling locked down strategies for example (Griette et al. 2020; Liu et al. 2020). More precisely, we will assume that there exists $T_{\text {lock }}$ and $\mu_{\text {lock }}$ such that the transmission rates can be written as

$$
\tau_{v}(t)=\left\{\begin{array}{cl}
\tau, & t \in\left[0, T_{\text {lock }}\right] \\
\frac{\tau \exp \left(-\mu_{\text {lock }}\left(t-T_{\text {lock }}\right)\right)+\tau_{\text {lock }}}{1+\exp \left(-\mu_{\text {lock }}\left(t-T_{\text {lock }}\right)\right)}, & t>T_{\text {lock }}
\end{array}\right.
$$

for each $v \in \mathcal{V}$ and for a given $0<\tau_{\text {lock }}<\tau$. We will assume that the four vertices are at equal distance such that $\ell_{e}=\ell$ for each $e \in \mathcal{E}$ and that the coefficient diffusion are equal on each edge, $d_{e}=d, e \in \mathcal{E}$. We further assume that at the central vertex $v_{2}$ exchanges are no longer allowed after locked down. That is, we impose that

$$
\alpha_{e}^{2}=\left\{\begin{array}{cl}
\alpha, & t \in\left[0, T_{\text {lock }}\right], \\
\alpha \exp \left(-\mu_{\text {lock }}\left(t-T_{\text {lock }}\right)\right), & t>T_{\text {lock }},
\end{array} \quad e \in \mathcal{E},\right.
$$

while $\alpha_{e}^{j}=\alpha$ for $j \neq 2$ and $e \in \mathcal{E}$, together with

$$
\lambda_{e}^{2}=\left\{\begin{array}{cl}
\lambda, & t \in\left[0, T_{\text {lock }}\right], \\
\lambda \exp \left(-\mu_{\text {lock }}\left(t-T_{\text {lock }}\right)\right), & t>T_{\text {lock }},
\end{array} \quad e \in \mathcal{E},\right.
$$

while $\lambda_{e}^{j}=\lambda$ for $j \neq 2$ and $e \in \mathcal{E}$, and also

$$
v_{e, e^{\prime}}^{2}=\left\{\begin{array}{cl}
v, & t \in\left[0, T_{\text {lock }}\right], \\
v \exp \left(-\mu_{\text {lock }}\left(t-T_{\text {lock }}\right)\right), & t>T_{\text {lock }},
\end{array} \quad\left(e, e^{\prime}\right) \in \mathcal{E} \times \mathcal{E} .\right.
$$

Finally, we set $\eta_{v}=\eta$ for all $v \in \mathcal{V}$. Regarding the initial condition, we work with

$$
\left(S_{1}^{0}, I_{1}^{0}, S_{2}^{0}, I_{2}^{0}, S_{3}^{0}, I_{3}^{0}, S_{4}^{0}, I_{4}^{0}\right)=\left(S_{0}-I_{0}, I_{0}, S_{0}, 0, S_{0}-\epsilon, 0, S_{0}+\epsilon, 0\right),
$$

for given $\left(S_{0}, I_{0}, \epsilon\right)$, while for each $e \in \mathcal{E}$ we have set $u_{e}^{0}(x)=0$ on $\Omega_{e}$. 

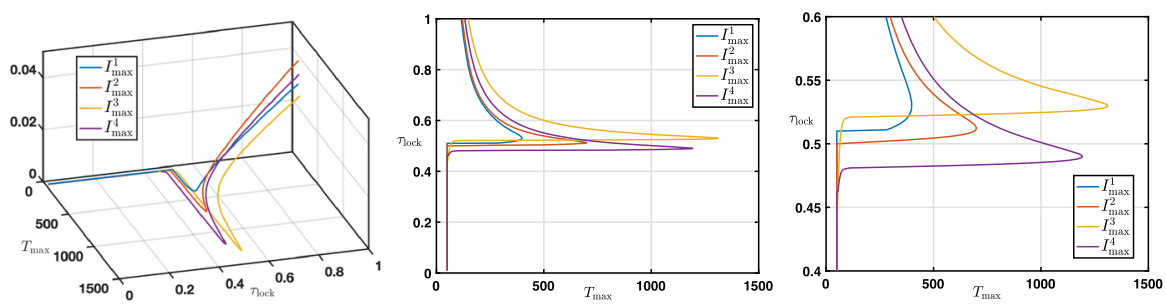

Fig. 14 Location of the time of maximal infection $T_{\max }$ for each vertex together with the corresponding amplitude $I_{\text {max }}^{j}$ as a function of $\tau_{\text {lock }}$ (left) with its projection in the $\left(T_{\max }, \tau_{l o c k}\right)$-plane (middle) and a zoom near the turning points (right). Other parameters are set to $\ell=1, d=0.1, \eta=1 / 8,(\alpha, \lambda, v)=$ $(1 / 8,1 / 20,1 / 20), T_{\text {lock }}=50$ and $\mu_{\text {lock }}=100$ with $\left(S_{0}, I_{0}, \epsilon\right)=\left(1 / 4,10^{-6}, 10^{-2}\right)$

In Fig. 14, we report the location of the time of maximal infection $T_{\max }$ for each vertex together with the corresponding amplitude $I_{\text {max }}^{j}=\max _{t \geq 0} I_{j}(t)$ as a function of $\tau_{\text {lock }}$. We observe that below a critical value of $\tau_{\text {lock }}$, the time of maximal infection always occurs at $t=T_{\max }=T_{\text {lock }}$ traducing the fact that the locked down strategy has no effect on the dynamics of the epidemic. At each vertex, we observe the same pattern: as $\tau_{\text {lock }}$ is decreased the corresponding $I_{\text {max }}^{j}$ is decreasing while $T_{\text {max }}$ is increasing up to some value of $\tau_{\text {lock }}$ where we observe a sudden turning point (see the right panel of Fig. 14). We observe that $\tau_{\text {lock, } v_{k}}^{t p}$, the value of the turning point, is well approximated (actually always bounded by below) by the value at which the effective reproduction number of each vertex is equal to 1 . Indeed we have $\mathscr{R}_{e, v_{k}}=1$ if and only if $\tau_{v_{k}}^{c}=\frac{\eta_{v_{k}}}{S_{v_{k}}^{0}}$, and we find

$$
\tau_{v_{1}}^{c} \simeq 0.5, \quad \tau_{v_{2}}^{c}=0.5, \quad \tau_{v_{3}}^{c} \simeq 0.52, \quad \text { and } \quad \tau_{v_{4}}^{c} \simeq 0.48
$$

with our specific values of the initial condition, while we have computed

$$
\tau_{l o c k, v_{1}}^{t p} \simeq 0.53, \quad \tau_{l o c k, v_{2}}^{t p} \simeq 0.51, \quad \tau_{\text {lock, } v_{3}}^{t p} \simeq 0.53, \quad \text { and } \quad \tau_{l o c k, v_{4}}^{t p} \simeq 0.49
$$

We also point out that when $\tau_{\text {lock }}$ is below the turning point $\tau_{l o c k, v_{k}}^{t p}$, the corresponding value of $I_{\text {max }}^{j}$ is below $10^{-3}$. On the other hand, in Fig. 15, we present similar results but this time $\tau_{\text {lock }}$ is fixed and $T_{\text {lock }}$ varies. Above some critical value of $T_{\text {lock }}$, $I_{\text {max }}^{j}$ saturates to a fixed value independent of $T_{\text {lock }}$ traducing the fact that the locked down strategy has no effect on the dynamics of the epidemic if it occurs to late in time. Depending on the initial configuration of susceptible populations at each vertex, we observe intricate nonlinear relationships on the location of the time of maximal infection $T_{\text {max }}$.

\subsection{Case of $N+1$ vertices and $N$ edges}

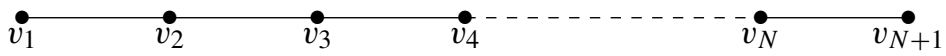



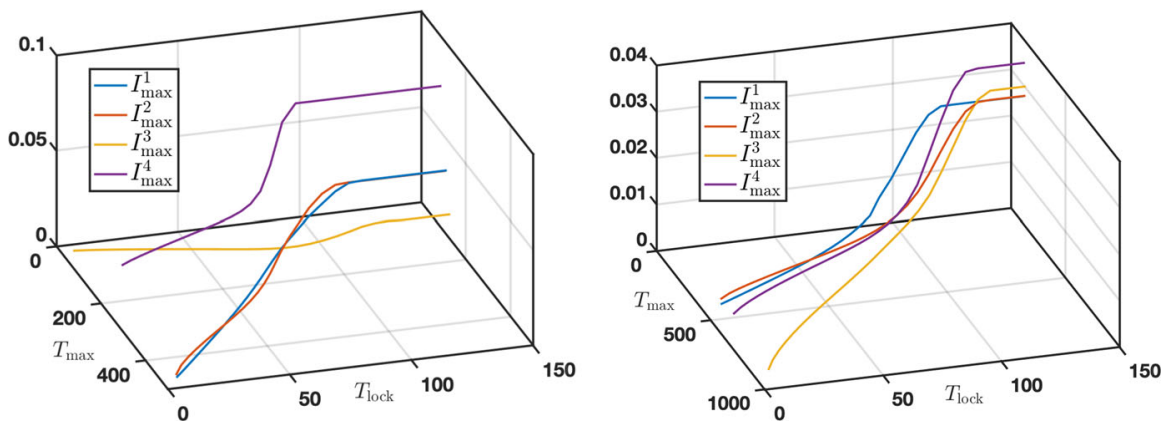

Fig. 15 Location of the time of maximal infection $T_{\max }$ for each vertex together with the corresponding amplitude $I_{\max }^{j}$ as a function of $T_{\text {lock }}$ for two configurations of initial susceptible populations at vertices $v_{3}$ and $v_{4}$, with $\epsilon=10^{-1}$ (left) and $\epsilon=10^{-2}$ (right). Other parameters are set to $\ell=1, d=0.1, \eta=1 / 8$, $(\alpha, \lambda, v)=(1 / 8,1 / 20,1 / 20), T_{\text {lock }}=50$ and $\eta_{\text {lock }}=0.6$ with $\left(S_{0}, I_{0}\right)=\left(1 / 4,10^{-6}\right)$

In our final example, we have considered a network of $N+1$ vertices and $N$ edges arranged in a lattice, in the sense that vertex $v_{j}$ is only connected to vertices $v_{j-1}$ and $v_{j+1}$ via two different edges. Figure 16 shows the time evolution of the infected population $I_{v_{j}}(t)$ and susceptible populations $S_{v_{j}}(t)$ at each vertex for several different initial conditions when the length and diffusion coefficient of each edge are equal. In the first case (top panel), we assume that $I_{v_{1}}^{0}>0$ while $I_{v_{j}}^{0}=0$ for all other vertices, and observe a propagation of burst of activity among infected and susceptible populations. In the second case (middle panel), we assume that $I_{v_{[N / 2]}}^{0}>0$ while $I_{v_{j}}^{0}=0$ for all other vertices, and we see the propagation of two bursts of activity among infected and susceptible populations going leftwards and rightwards. In the last case (bottom panel), we assume that $I_{v_{1}}^{0}=I_{v_{N+1}}^{0}>0$ while $I_{v_{j}}^{0}=0$ for all other vertices, and we note the propagation of two waves activity which collide at the middle vertex $v_{\lfloor N / 2\rfloor}$. For very small values of the diffusion coefficient $d$, this burst of epidemic activity seems to travel coherently and forms a coherent traveling wave, as can be seen in Fig. 17 where we represent the location of $\max _{t>0} I_{v_{j}}(t)$ at each vertex. Such a traveling wave of epidemic activity share similarities with traveling waves in excitable media such as the propagation of electrical activity along a nerve cell (Hodgkin and Huxley 1952; Hupkes and Sandstede 2010) or calcium waves Sneyd (2005). When $d=10^{-3}$, they are all aligned on the same line, where for smaller values $d \in\left\{10^{-1}, 10^{-2}\right\}$ the location is a nonlinear curve. We also demonstrate that larger diffusion coefficient leads to a faster propagation of epidemic burst across vertices. Finally, we also remark that if $I_{\max , 1}^{d}$ denotes the maximum as a function of $d$ at the first vertex, we have $I_{\max , 1}^{d_{1}} \leq I_{\max , 1}^{d_{2}}$ for $d_{1} \leq d_{2}$ while for larger vertices $j \geq 6$ we have the reverse ordering $I_{\max , j}^{d_{1}} \geq I_{\max , j}^{d_{2}}$ for $d_{1} \leq d_{2}$.

For the numerical simulations presented in Figs. 16 and 17, we have assumed full symmetry in the parameters that is

$$
\begin{aligned}
& \left(\ell_{e}, d_{e}\right)=(\ell, d), \quad e \in \mathcal{E}, \quad\left(\tau_{v}, \eta_{v}\right)=(\tau, \eta), \quad v \in \mathcal{V}, \\
& \left(\alpha_{e}^{v}, \lambda_{e}^{v}\right)=(\alpha, \lambda), \quad(e, v) \in \mathcal{E} \times \mathcal{V}, \quad v_{e, e^{\prime}}^{v}=v, \quad\left(e, e^{\prime}, v\right) \in \mathcal{E} \times \mathcal{E} \times \mathcal{V} .
\end{aligned}
$$



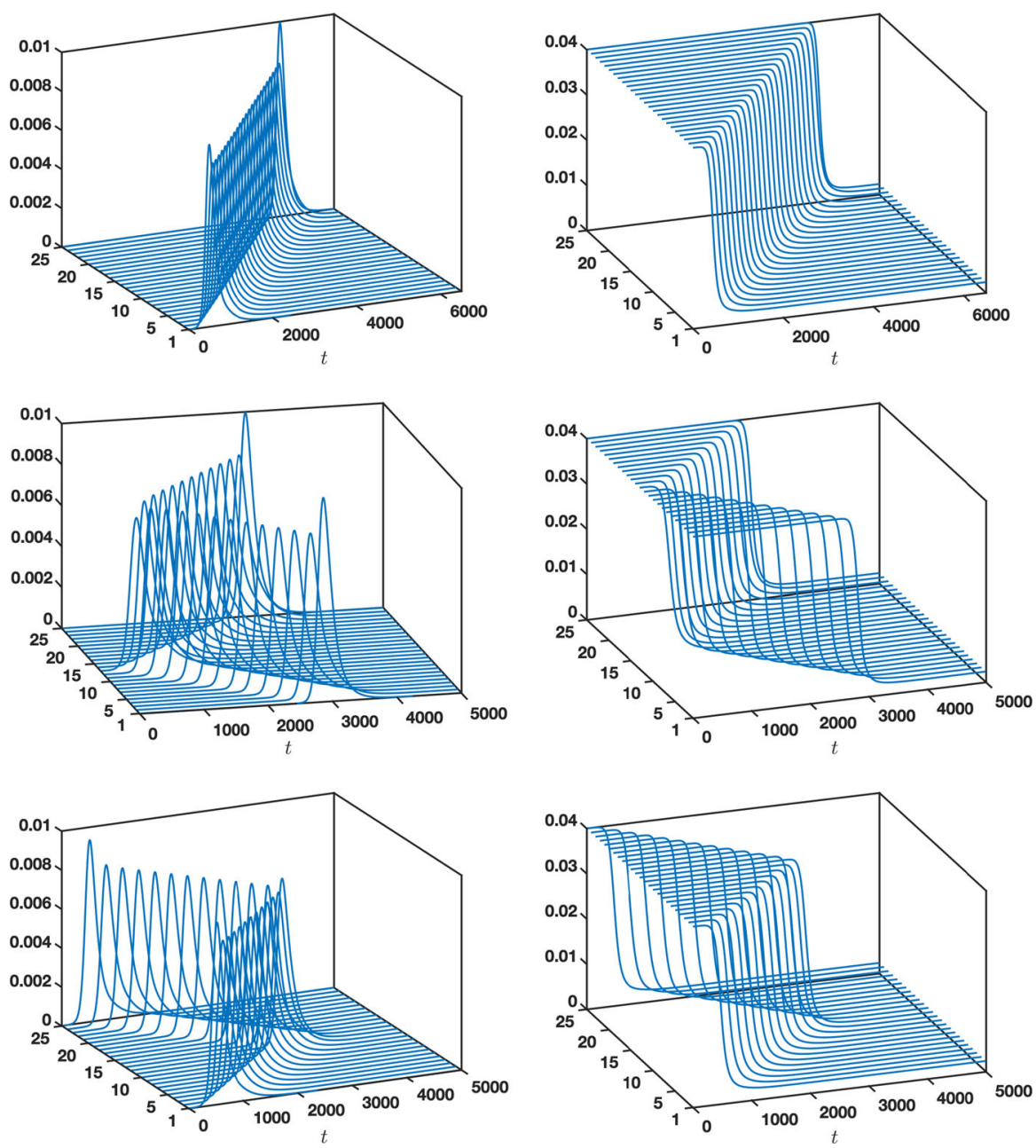

Fig. 16 Time evolution of the infected (left) population $I_{j}(t)$ and susceptible (right) populations $S_{j}(t)$ at each vertex for several different initial conditions and $N=24$. Top: infected individuals are initially present only at vertex. Middle: infected individuals are initially present only at the middle vertex. Bottom: infected individuals are initially present only at the first and last vertices. We observe a traveling wave of infectious activity propagating though the vertices. Parameters were set to $\ell=1, d=10^{-3},(\tau, \eta)=(1,1 / 75)$, and $(\alpha, \lambda, v)=(1 / 8,1 / 10,1 / 20)$, while the initial condition is $\left(S_{0}, I_{0}\right)=\left(1 / 25,10^{-6}\right)$

Regarding the initial condition on the edge, we have set $u_{e}^{0}(x)=0$ on $\Omega_{e}$ for each $e \in \mathcal{E}$.

\section{Discussion}

\subsection{Summary of main results}

In this work, we have proposed a new model that describes the dynamics of epidemic spreading on connected graphs. Our model consists in a PDE-ODE system where at 

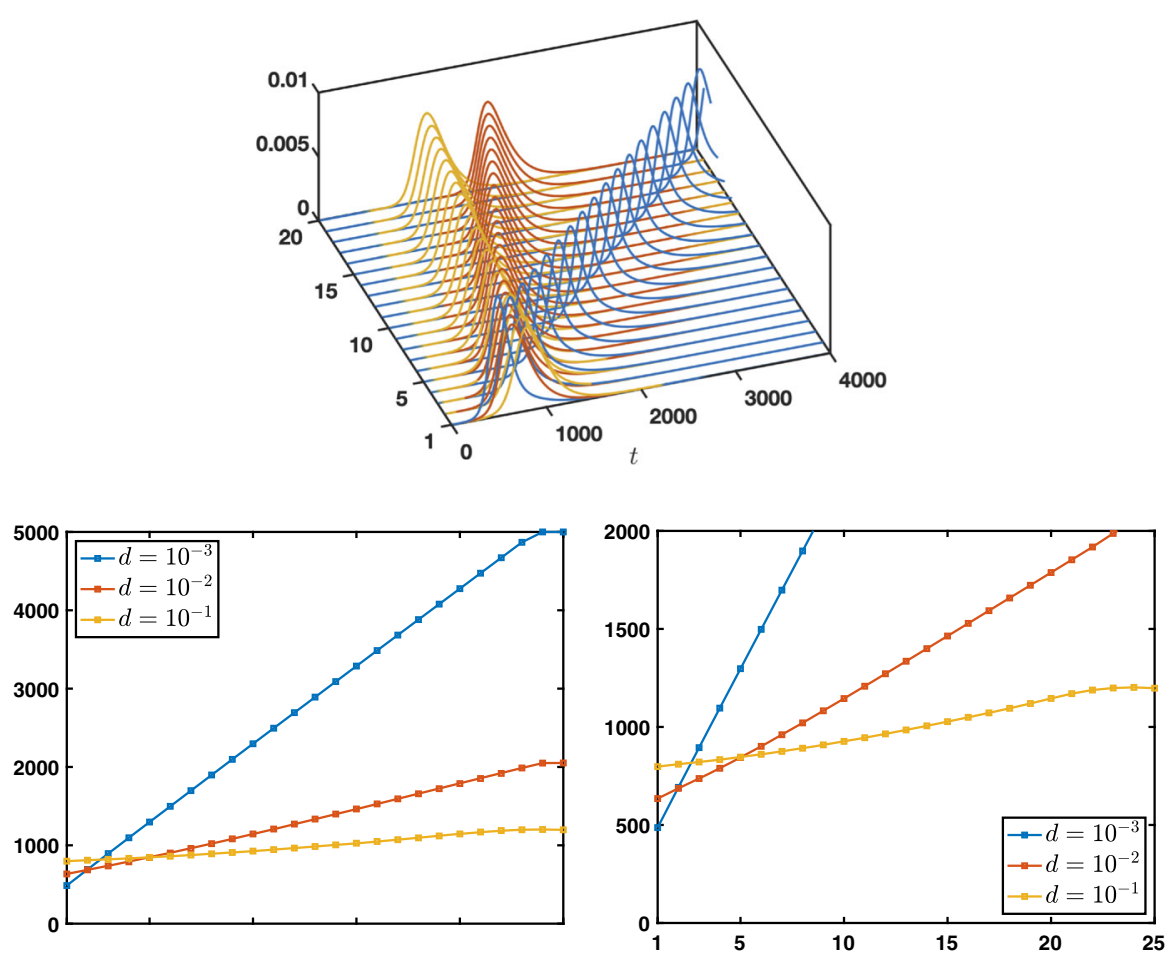

Fig. 17 Left: Location of the maxima of the infected population $I_{j}(t)$ at each vertex $j \in\{1, \ldots, 25\}$ for several different diffusion coefficients $d \in\left\{10^{-3}, 10^{-2}, 10^{-1}\right\}$. Right: Zoom of the left figure for small times. We observe that for very small $d$ the location of the maxima is a long a line while it is curved for larger values of $d$. We also remark that if $I_{\max , 1}^{d}$ denotes the maximum as a function of $d$ at the first vertex, we have $I_{\max , 1}^{d_{1}} \leq I_{\max , 1}^{d_{2}}$ for $d_{1} \leq d_{2}$ while for larger vertices $j \geq 6$ we have the reverse ordering $I_{\max , j}^{d_{1}} \geq I_{\max , j}^{d_{2}}$ for $d_{1} \leq d_{2}$. Other parameters were set to $\ell=1,(\tau, \eta)=(1,1 / 75)$, and $(\alpha, \lambda, v)=(1 / 8,1 / 10,1 / 20)$, while the initial condition is $\left(S_{0}, I_{0}\right)=\left(1 / 25,10^{-6}\right)$

each vertex of the graph we have a standard SIR model and connections between vertices are given by heat equations on the edges supplemented with Robin like boundary conditions at the vertices modeling exchanges between incident edges and the associated vertex. Our first main result is the existence and uniqueness of classical, global in time, solutions of our PDE-ODE model. Our second main result is a complete characterization of the long time behavior of the unique solution of our model. We proved that the final total populations of infected individuals at each vertex are well defined quantities and solutions of a system implicit equations. We also managed to obtain further qualitative properties in the fully symmetric case by exhibiting closed form formula and relate these quantities to standard basic and effective reproductive number for classical SIR model. Next, we proposed and analyzed a semi-implicit in time numerical scheme based on finite differences in space which has the property to preserve a discrete total mass associated to the discretization. We have further proved that if the time discretization constant is smaller than a universal constant depending 
only on the parameters of the system (and not on the space discretization constant) and if the exchanges of fluxes are symmetric at each vertex, then our mass preserving semi-implicit numerical scheme is well-posed and preserves the positivity of the solutions. And finally, we illustrated our theoretical findings with selection of numerical simulations for various types of graphs showing very interesting dynamics such traveling waves.

\subsection{Biological limitations of the model and possible extensions}

The first biological limitation of our model comes from the purely diffusive behavior imposed on each edge of the graph. It would be biologically relevant to incorporate some kind of directed or ballistic motion (drift term in (2.2)) given that many individuals set off on transportation lines travel from one specific city to another preferentially, see Bertaglia and Pareschi (2021) for a purely hyperbolic model. This would result in an equation on each edge of the form

$$
\partial_{t} u_{e}(t, x)=d_{e} \partial_{x}^{2} u_{e}(t, x)+c_{e} \partial_{x} u_{e}(t, x), \quad t>0, \quad x \in \stackrel{\circ}{\Omega}_{e}
$$

for some speed $c_{e} \in \mathbb{R}$ whose sign will determine the preferred direction of transportation. Such a drift term modifies the boundary conditions at each vertex according to

$$
D_{v} \partial_{n} \mathbf{u}_{v}(t)+\widetilde{K}_{v} \mathbf{u}_{v}(t)=\Lambda_{v} \mathbf{I}_{v}(t),
$$

where $\widetilde{K}_{v}$ is given by $\widetilde{K}_{v}=K_{v}-C_{v}$ where $C_{v}=\operatorname{diag}\left[\left(c_{e}\right)_{e \sim v}\right]$. We expect that the long time dynamics of the system with such a directed motion, which is still mass preserving, will be somehow similar to the purely diffusive case, and that only transient dynamics will be affected. However, we leave it as an open modeling problem at the moment.

A second biological limitation comes from our assumption that the movement of individuals in the susceptible population does not affect its distribution and thus only the infected population is subject to movement. Considering the susceptible population as an ambient population was a first step, and it would be natural to extend our model to the case that individuals in the susceptible population can also move along our transportation network. In the case of spatially extended systems of reactiondiffusion type, it is notorious that allowing the susceptible population to diffuse is more challenging from a theoretical point of view as monotonicity properties of the solutions are lost (Berestycki et al. 2020). We leave such an analysis for a future work.

\subsection{Scaling limits}

Several scaling limits could be considered and we present two directions which seem natural and very promising. First, it would be very interesting to investigate the limit of large diffusivity along the edges of the graph. One should be able to recover a class of network-based ODE models that have been studied in the literature. Indeed, we 
expect that in this limit, one should obtain an ODE for some $U_{e}(t)$ (now independent of $x \in \stackrel{\circ}{\Omega}_{e}$ ) along the edges that depends only on the population levels at the two connecting nodes. This should lead to an ODE system at the vertices of the graph that is globally coupled to $\left(U_{e}\right)_{e \in \mathcal{E}}$.

Another natural limit to investigate is the one where the number of vertices of the graph goes to infinity along with the length of the edges which shall converge to zero. One would expect to obtain in the limit a PDE system describing the evolution of spatially continuous populations of susceptible, infected and removed populations. Depending on the initial geometry of the graph the limiting PDE will either be posed on the real line or on some two-dimensional domain. The exchange term between infected individuals at the edges, given by $\sum_{e \sim v} \alpha_{e}^{v} u_{e}(t, v)-\bar{\lambda}_{v} I_{v}(t)$, is likely to produce in the limit a spatial diffusion term and we expect to recover reaction-diffusion like models such as the ones in Aronson (1977), Berestycki et al. (2020) and Berestycki et al. (2020).

Acknowledgements This works was partially supported by Labex CIMI under Grant Agreement ANR11-LABX-0040. G.F. acknowledges support from an ANITI (Artificial and Natural Intelligence Toulouse Institute) Research Chair. We thank Philippe Laurençot for his astute comments on an earlier version of the manuscript.

\section{References}

Aronson DG (1977) The asymptotic speed of propagation of a simple epidemic. Res Notes Math 14:1-23 Ball F, Britton T (2020) Epidemics on networks with preventive rewiring. arXiv preprint arXiv:2008.06375 Berestycki H, Roquejoffre J-M, Rossi L (2020) Propagation of epidemics along lines with fast diffusion. Bull Math Biol 83:1-34

Berestycki H, Nordmann S, Rossi L (2020) Modeling propagation of epidemics, social unrest and other collective behaviors. arXiv preprint arXiv:2005.09865

Bertaglia G, Pareschi L (2021) Hyperbolic models for the spread of epidemics on networks: kinetic description and numerical methods. ESAIM Math Model Numer Anal 55:381-407

Bonnasse-Gahot L, Berestycki H, Depuiset M-A, Gordon MB, Roché S, Rodriguez N, Nadal J-P (2018) Epidemiological modelling of the 2005 French riots: a spreading wave and the role of contagion. Sci Rep 8:1-20

Britton T, Deijfen M, Lindholm M, Nordvall LA (2008) Epidemics on random graphs with tunable clustering. J Appl Probab 45:743-756

Centers for Disease Control and Prevention (2003) Severe acute respiratory syndrome-Singapore, 2003. Morb Mortal Wkly Rep 52(18):405

Corless RM, Gonnet GH, Hare DE, Jeffrey DJ, Knuth DE (1996) On the LambertW function. Adv Comput Math 5(1):329-359

David JF, Iyaniwura SA, Ward MJ, Brauer F (2020) A novel approach to modelling the spatial spread of airborne diseases: an epidemic model with indirect transmission. Math Biosci Eng 17(4):3294

Diekmann O (1978) Thresholds and travelling waves for the geographical spread of infection. J Math Biol 6:109-130

Diekmann O, Heesterbeek JAP, Metz JAJ (1990) On the definition and the computation of the basic reproduction ratio $R 0$ in models for infectious diseases in heterogeneous populations. J Math Biol 28:365

Gou J, Ward MJ (2016) Oscillatory dynamics for a coupled membrane-bulk diffusion model with FitzhughNagumo membrane kinetics. SIAM J Appl Math 76(2):776-804

Gou J, Li YX, Nagata W, Ward MJ (2015) Synchronized oscillatory dynamics for a 1-D model of membrane kinetics coupled by linear bulk diffusion. SIAM J Appl Dyn Syst 14(4):2096-2137

Griette Q, Magal P, Seydi O (2020) Unreported cases for age dependent COVID-19 outbreak in Japan. Biology 9:132 
Hethcote HW (2000) The mathematics of infectious diseases. SIAM Rev 42(4):599-653

Hodgkin AL, Huxley AF (1952) A quantitative description of membrane current and its application to conduction and excitation in nerve. J Physiol 117:500-544

Hupkes HJ, Sandstede B (2010) Traveling pulse solutions for the discrete FitzHugh-Nagumo system. SIAM J Appl Dyn Syst 9(3):827-882

Kermack WO, McKendrick AG (1927) A contribution to the mathematical theory of epidemics. Proc R Soc Ser A 115:700-721

Liu Z, Magal P, Seydi O, Webb G (2020) Predicting the cumulative number of cases for the COVID-19 epidemic in China from early data. Math Biosci Eng 17(4):3040-3051

Magal P, Seydi O, Webb G (2018) Final size of a multi-group SIR epidemic model: irreducible and nonirreducible modes of transmission. Math Biosci 301:59-67

Magal P, Seydi O, Webb G (2016) Final size of an epidemic for a two group SIR model. SIAM J Appl Math 76:2042-2059

Mandal S, Sarkar RR, Sinha S (2011) Mathematical models of malaria-a review. Malar J 10(202):1-19

Magal P, Webb G (2018) The parameter identification problem for SIR epidemic models: identifying unreported cases. J Math Biol 77(6-7):1629-1648

New England Journal of Medicine (2020) Letter to the Editor. https://doi.org/10.1056/NEJMc2001468, January 30, 2020

Paquin-Lefebvre F, Nagata W, Ward MJ (2020) Weakly nonlinear theory for oscillatory dynamics in a one-dimensional PDE-ODE model of membrane dynamics coupled by a bulk diffusion field. SIAM J Appl Math 80(3):1520-1545

Reluga T (2004) A two-phase epidemic driven by diffusion. J Theor Biol 229(2):249-261

Sahneh FD, Scoglio C, Van Mieghem P (2013) Generalized epidemic mean-field model for spreading processes over multilayer complex networks. IEEE/ACM Trans Netw 21(5):1609-1620

Sekiguchi M, Emiko I (2011) Dynamics of a discretized SIR epidemic model with pulse vaccination and time delay. J Comput Appl Math 236(6):997-1008

Sneyd J (2005) Tutorials in mathematical biosciences II. Lecture notes in mathematics, Chapter Mathematical modelling of calcium dynamics and signal transduction, vol 187. Springer, New York

Spricer K, Britton T (2019) An SIR epidemic on a weighted network. Netw Sci 7:556-580

Stolerman LM, Coombs D, Boatto S (2015) SIR-network model and its application to dengue fever. SIAM J Appl Math 75(6):2581-2609

Van den Driessche P, Watmough J (2002) Reproduction numbers and sub-threshold endemic equilibria for compartmental models of disease transmission. Math Biosci 180(1-2):29-48

Publisher's Note Springer Nature remains neutral with regard to jurisdictional claims in published maps and institutional affiliations. 